Problema restrito dos três corpos

Fernando Pereira Micena 
SERVIÇO DE PÓS-GRADUAÇÃO DO ICMC-USP

Data de Depósito: 16 de janeiro de 2007

Assinatura:

\section{Problema restrito dos três corpos}

Fernando Pereira Micena

Orientador: Prof. Dr. Ali Tahzibi

Dissertação apresentada ao Instituto de Ciências Matemáticas

e de Computação - ICMC/USP, como parte dos requisitos para obtenção do título de Mestre em Matemática.

USP - São Carlos

Janeiro/2007 
Aos que mais amo, minha família. 


\section{Agradecimentos}

Agradeço primeiramente a Deus. Puro atrevimento meu em dirigir-lhe a palavra, pois diante Dele não tenho a dignidade que me permita fazê-lo. Não há palavras que possam descrever o quão sou infinitamente grato, mas tenho a certeza que eu o terei agradecido mais de uma vez, ao expor a minha gratidão àqueles que em minha vida concretizaram Deus em suas ações.

Agradeço aos que mais amo, minha família. Aos meus pais, José e Dirce, agradeço. Se pudesse e me fosse cabido o poder para tal, declararia-os doutores na arte de amar, educar e criar um filho. De tudo que sou hoje, boa parcela, senão tudo, devo a eles. Peço emprestado os seguintes versos para dedicar a minha mãe:

\section{"Está sempre me ensinando um caminho, não o lógico, para comigo é só coração, ensina-me através do carinho o que às vezes aprendo sozinho por não lhe dar atenção. "}

Agradeço aos meus irmãos, Marcelo e Raul. Agradeço-lhes pela força e amizade, sempre tenho boas lições de vida a aprender com ambos. À Cássia que já considero parte da minha família, sinta-se inclusa em todas as citações que faço da palavra: família. A você agradeço sua amizade, amor e companheirismo. Quando não tinha o meu lar para me abrigar era em seus braços que procurava refúgio, que nunca me faltou.

Um agradecimento aos meus amigos, sintam-se todos abraçados. Mas não poderia deixar de citar alguns nomes em especial. Fabrício: pela amizade duradoura, longas, animadas, profundas e enriquecedoras conversas. Nivaldo e Esdras: pela amizade e grande força no ínicio de minha caminhada em São Carlos.

Não poderia esquecer de agradecer aos educadores, professores e funcionários, das escolas em que estudei até a faculdade e o mestrado. Agradeço a todos eles. Aqui também gostaria de mencionar alguns nomes, a começar por duas professoras que tiveram que ter muita paciência comigo: Tia Ana Maria (Tiana), professora do pré, e Tia Leonor, professora da primeira série. Obrigado pelo alicerce. Agradeço a Francisco Chubaci (Chicão), graças a ele é que optei pela 
matemática depois de ler o livro: "O Romance das Equações Algébricas", que ele me emprestou, e é claro um agradecimento também pela amizade e o préstimo. Da faculdade não poderia deixar de fazer um agradecimento mais que especial a Paulo Ricardo, Cláudio Buzzi e a Professora Neuza, por todos os ensinamentos, confiança, paciência e a amizade.

Agradeço ao Professor Ali Tahzibi, pela orientação, pelo seu entusiasmo, conhecimentos ensinados, a força que me passou nas horas difíceis do mestrado e sobretudo pela amizade.

A todos os professores e funcionários da USP os meus sinceros agradecimentos.

Por fim um agradecimento oficial: à FAPESP, pelo fomento. 


\begin{abstract}
The $n$-body problem is one of the most important problems in dynamical systems. We study the model introduced by Sitnikov of restricted three body problem. In this model the primaries are of equal mass and the third body is very small with respect to the primaries. Using methods of Alekseev, we show the existence of "Smale horseshoe" as a subsystem of the dynamic of the third body and conclude rich probabilistic consequences. We also study the same problem by Melnikov's method.
\end{abstract}

Key Words: Smale horseshoe, Symbolic Dynamic, Bernoulli's shift. 


\section{Resumo}

O problema de $n$ - corpos é um dos problemas mais importantes em Sistemas Dinâmicos. Nós estudamos o modelo do problema dos três corpos restrito introduzido por Sitnikov. Nesse modelo os corpos primários tem a mesma massa e o terceiro corpo é de massa muito pequena com respeito aos corpos primários. Usando os métodos de Alekseev, nós mostramos a existência de uma "ferradura de Smale" como um subsistema da dinâmica do terceiro corpo e concluímos ricas conseqüências probabilísticas. Nós também estudamos o problema pelo método de Melnikov.

Palavras Chave: Ferradura de Smale, Dinâmica Simbólica, Shift de Bernoulli. 


\section{Conteúdo}

1 Breve Introdução à Dinâmica Simbólica 11

1.1 O Shift como Subsistema . . . . . . . . . . . . . . . . . 11

1.2 Condições Alternativas para aplicações $\phi$ de Classe $C^{1}$. . . . . . . . . . . . . 21

1.3 Estrutura Hiperbólica do Conjunto Invariante . . . . . . . . . . . . . . . . . . . 24

2 Estudo Qualitativo e Probabilístico do Problema dos Três Corpos 31

2.1 Formulação do Problema . . . . . . . . . . . . . . . . . . . . . 31

2.2 Propriedades Gerais das Soluções da Equação $\ddot{z}=-Q(z, t)$. . . . . . . . . . . 33

2.3 Estudo Probabilístico do Problema dos Três Corpos . . . . . . . . . . . . 50

3 Nascimento da Ferradura de Smale $\quad 60$

3.1 Uma Visão Geométrica do Problema Restrito dos Três Corpos . . . . . . . . . . 60

3.1.1 Método de Melnikov para Pontos Homoclínicos de Sistemas Hamiltoni-

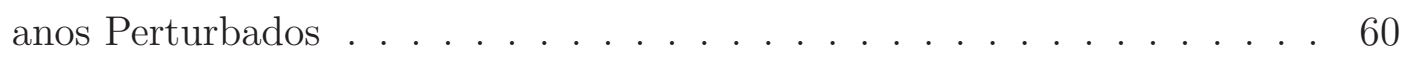

3.2 Existência de Pontos Homoclínicos Transversais no Problema de Sitnikov . . . . 65

3.2.1 As Transformações de McGehee . . . . . . . . . . . . . . . . 67

3.2.2 Método de Melnikov aplicado ao Problema Restrito dos Três Corpos . . . 70

3.3 A Dinâmica $S$ Possui um Shift como subsistema . . . . . . . . . . . . . . . 72

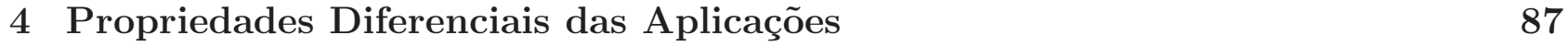

4.1 Propriedades Diferenciais das Aplicações $Z^{ \pm}, T^{ \pm}, h^{ \pm} \ldots \ldots$. . . . . . . . . . 87

4.2 Propriedades Diferenciais da Aplicação S. . . . . . . . . . . . . . . . . . 105 


\section{Introdução}

Dentre as várias áreas em que a matemática encontra aplicação, a mecânica celeste ocupa excepcional posição. Por mais de 300 anos, problemas em mecânica celeste, especialmente o problema dos três corpos, vinha servindo como desafio aos matemáticos por gerações. As tentativas de resolver o problema legaram resultados muito importantes, contribuindo para o avanço da matemática.

A cerca de um século atrás Henri Poincaré em seu "Méthods nouvelles de la mécanique celeste" apresenta resultados que dão grande estímulo a novos estudos em mecânica celeste. Poincaré em seu trabalho apresenta novas idéias e abordagem do problema. Conceitos tais como: método dos pequenos parâmetros, séries assintóticas, investigação de órbitas periódicas, curvas homoclínicas, integrais invariantes, e em outras palavras, boa parte do que é chamada hoje de Teoria Qualitativa das Equações Diferenciais, são apresentados neste trabalho.

Após o trabalhos de Poincaré, as ferramentas introduzidas por ele, são amplamente utilizadas nos estudos de problemas em mecânica celeste. No diz respeito às intersecções homoclínias, podemos encontrar dinâmicas descritas por Smale, as Ferraduras de Smale, e a partir daí obter informações qualitativas do problema, via Dinâmica Simbólica.

Em uma nova formulação do problema de três corpos, apresentada Sitnikov, desenvolveremos o nosso trabalho. Queremos, a partir de ferramentas de Dinâmica Hiperbólica, descrever a dinâmica do problema, através da Dinâmica Simbólica. Dois pontos de vista serão abordados: o ponto de vista de Moser e o de Alekseev. Como veremos o ponto de vista de Moser é mais geométrico. Sob esta análise, mudamos as coordenadas do sistema original pelas coordenadas de McGehee, afim de obtermos um campo vetorial em $\mathbb{R}^{2}$ tal que a origem tem comportamento geométrico de ponto de sela.

A análise feita por Alekseev aborda o problema de maneira mais geral, considerando uma classe de equações, freqüentemente associados à oscilações periódicas, e também na formulação do problema de três corpos devida a Sitnikov.

Nós reduziremos o problema a uma aplicação $S$ no plano, a qual desejamos mostrar que possui uma dinâmica de Ferradura de Smale.

Esta dissertação de divide em quatro capítulos. O primeiro deles é destinado ao estudo de 
uma aplicação

$$
\phi: Q \rightarrow \mathbb{R}^{2}
$$

onde $Q$ é o quadrado $[0,1] \times[0,1]$. Estabelecemos condições no caso em que $\phi$ é de classe $C^{1}$, a fim de obtermos em $Q$ um conjunto invariante $I$, sobre o qual a dinâmica $\phi$ restrita a $I$ seja conjugada a um Shift de Bernoulli.

No segunda capítulo, tratamos da formulação do problema de três corpos, conforme a configuração sugerida por Sitnikov. Após isso, abordamos uma classe de equações mais amplas

$$
\ddot{z}=-Q(z, t)
$$

e estudamos sobre esta classe informações qualitativas sobre o movimento associado, que é a solução $z(t, v, \tau)$ da equação diferencial ordinária dada acima. Dessa forma, foi possível dividir as soluções em quatro classes distintas. Neste capítulo também discutimos os aspectos probabilísticos do problema de três corpos e concluímos com o enunciado da conjecutura de Kolmogorov.

No capítulo seguinte, discutimos o surgimento da ferradura de Smale, para a transformação de Poincaré $S$ associada ao problema dos três corpos restrito. Sem muito rigor, fizemos o estudo da fórmula de Melnikov. Após isso utilizamos os resultados dos capítulos 1 e 4 para provar que a dinâmica $S$, definida num subconjunto das condições iniciais do plano $(v, \tau)$, tem um shift de $n$ símbolos. O número natural $n$ pode ser escolhido da forma que desejarmos.

No quarto e último capítulo da presente dissertação, demonstramos os resultados mais técnicos, utilizados no capítulo anterior. Neste capítulo, fizemos um estudo detalhado sobre as classes de diferenciabilidade das aplicações definidas nos capítulos precedentes. 


\section{Breve Introdução à Dinâmica Simbólica}

\subsection{O Shift como Subsistema}

Seja $\phi:[0,1] \times[0,1] \rightarrow \mathbb{R}^{2}$ uma aplicação contínua. Por simplicidade, denotaremos $Q=$ $[0,1] \times[0,1]$. Suponha que $\phi(Q) \cap Q$ intersecte $Q$ em duas componentes conexas disjuntas $V_{1}$ e $V_{2}$ como abaixo.

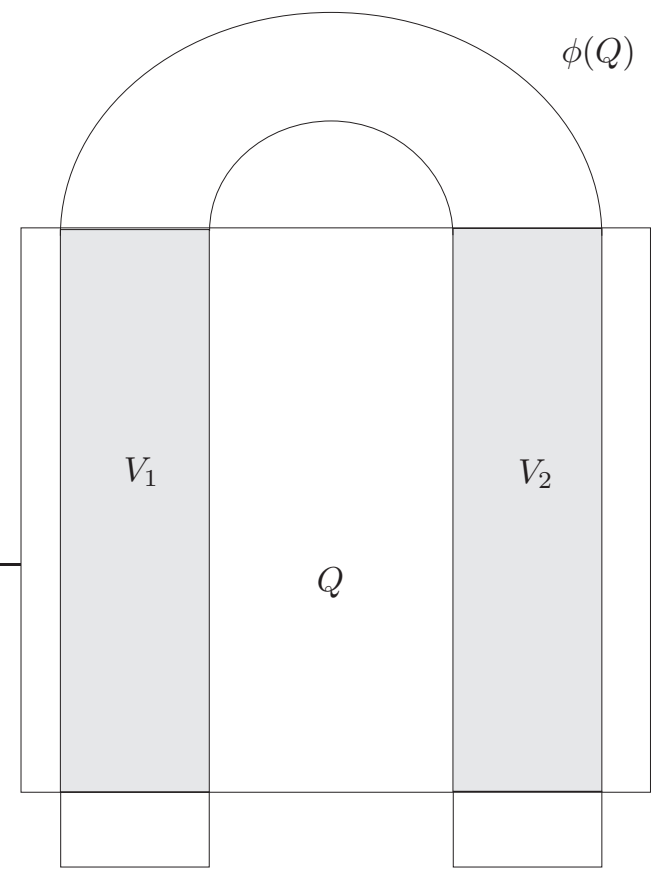

Figura 1: Ferradura Clássica

Nos interessa estudar a dinâmica $\phi$ o seguinte conjunto de pontos

$$
I=\bigcap_{n \in \mathbb{Z}} \phi^{n}(Q) .
$$

A dinâmica de $\phi$ sobre o conjunto $I$ é bastante conhecida no estudo de Sistemas Dinâmicos, trata-se da dinâmica de ferradura de Smale. No caso particular de $\phi(Q) \cap Q$ decompor o quadrado $Q$ em duas componentes conexas disjuntas, ou número finito delas o conjunto $I$ é um conjunto de Cantor.

Seja $A \subset \mathbb{N}$, não vazio, podendo conter infinitos elementos. Denote por $\Sigma_{A}$ o conjunto das seqüências bi-infinitas com elementos em A. 


$$
s=\left(\ldots s_{-2}, s_{-1}, s_{0}, s_{1}, s_{2}, \ldots\right)
$$

Introduzimos em $\Sigma_{A}$ a seguinte métrica $d: \Sigma_{A} \times \Sigma_{A} \rightarrow \mathbb{R}^{+}$

$$
d(s, t)=\sum_{i=-\infty}^{+\infty} \frac{\delta_{i}\left(s_{i}, t_{i}\right)}{2^{|i|}}
$$

onde

$$
\delta_{i}=\left\{\begin{array}{l}
\delta_{i}\left(s_{i}, t_{i}\right)=1 \quad \text { se } s_{i} \neq t_{i} \\
\delta_{i}\left(s_{i}, t_{i}\right)=0 \quad \text { se } s_{i}=t_{i}
\end{array}\right.
$$

No caso $A$ finito, $\left(\Sigma_{A}, d\right)$ é um espaço métrico completo, na verdade é mais, é um conjunto

\section{de Cantor.}

Seja $\sigma: \Sigma_{A} \rightarrow \Sigma_{A}$ satisfazendo

$$
\sigma\left(\left(s_{k}\right)_{k \in \mathbb{Z}}\right)=\left(s_{k-1}\right)_{k \in \mathbb{Z}}
$$

a aplicação $\sigma$ é chamada de função deslocamento ou shift .

No caso da ferradura clássica, o que temos é um conjugação topológica entre a dinâmica de $\phi$ sobre $I$ e o shift no espaço das seqüênicia bi-infinitas de dois símbolos, $\Sigma_{A}, A=\{1,2\}$.

Veremos a partir de agora condições para que uma aplicação $\phi: Q \rightarrow \mathbb{R}^{2}$ deixe um conjunto invariante $I \subset Q$, e a dinâmica $\phi \mid I$ seja conjugada com um shift num espaço de seqüências bi-infinitas, $\Sigma_{A}$, com $A$ finito. Queremos encontrar condições para que $\phi$ possua um shift como subsistema.

Definição 1.1 (Curva Horizontal). Seja $\mu<1$, nós chamamos uma curva $y=u(x)$ uma curva horizontal se $0 \leq u(x) \leq 1$ para $0 \leq x \leq 1$ e

$$
\left|u\left(x_{1}\right)-u\left(x_{2}\right)\right| \leq \mu\left|x_{1}-x_{2}\right|, \quad 0 \leq x_{1}, x_{2} \leq 1
$$

Definição 1.2 (Curva Vertical). Seja $\mu<1$, nós chamamos uma curva $x=v(y)$ uma curva horizontal se $0 \leq v(y) \leq 1$ para $0 \leq y \leq 1 e$

$$
\left|v\left(y_{1}\right)-v\left(y_{2}\right)\right| \leq \mu\left|y_{1}-y_{2}\right|, \quad 0 \leq y_{1}, y_{2} \leq 1
$$


Sejam $u_{1}$ e $u_{2}$ duas curvas horizontais, o conjunto

$$
U=\left\{(x, y) \mid 0 \leq x \leq 1 ; u_{1}(x) \leq y \leq u_{2}(x)\right\}
$$

é chamado de faixa horizontal.

Analogamente definimos uma faixa vertical $V$.

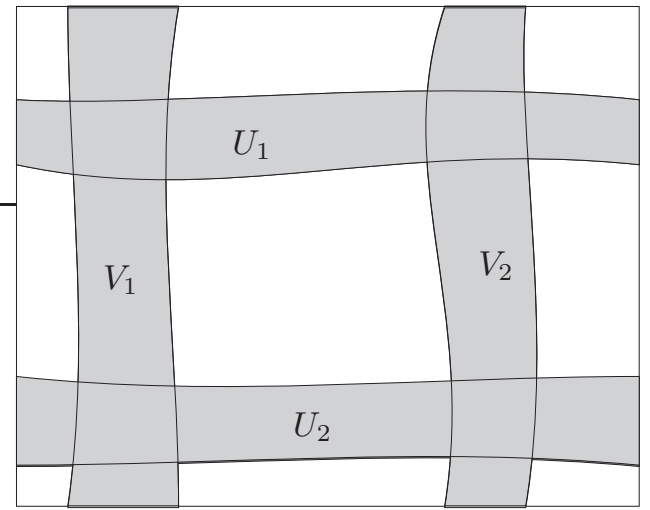

Figura 2: faixas horizontais e verticais

Dada uma faixa horizontal $U$, cuja fronteira é dada pelo gráfico das funções $u_{1}(x)$ e $u_{2}(x)$ como acima, definimos o número

$$
d(U)=\max _{0 \leq x \leq 1}\left(u_{2}(x)-u_{1}(x)\right)
$$

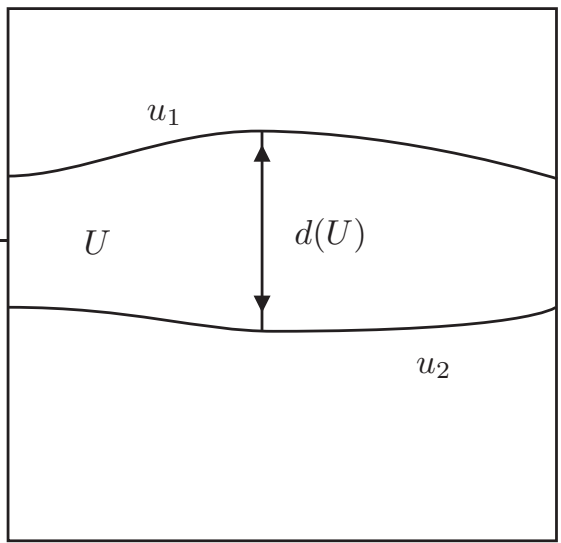

Figura 3: diâmetro da faixa 
que é chamado o diâmetro de $U$. Também de maneira análoga definimos o número $d(V)$ para faixas verticais.

Com respeito às faixas verticais e horizontais temos as seguintes propriedades:

Lema 1.1. Se $U^{(1)} \supset U^{(2)} \supset U^{(3)} \supset \ldots \supset U^{(n)} \supset \ldots$ é uma seqüência de faixas horizontais e se $d\left(U^{(k)}\right) \rightarrow 0$ quando $k \rightarrow \infty$ então

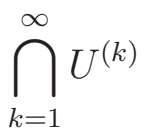

define uma curva horizontal.

Demonstração. Primeiro tomemos um valor $x_{0} \in[0,1]$ Consideremos então a família de compactos

$$
U^{i}\left(x_{0}\right)=U^{i} \cap\left\{x_{0} \times[0,1]\right\}, i=1,2, \ldots
$$

Como a seqüência dos compactos $U^{i}$ é encaixada, então $U^{i}\left(x_{0}\right)$ é encaixada e como $U^{i}\left(x_{0}\right) \subset U^{i}$ segue que

$$
d\left(U^{i}\left(x_{0}\right)\right) \rightarrow 0
$$

Daí temos que a intersecção

$$
\bigcap_{i=1}^{+\infty} U^{i}\left(x_{0}\right)
$$

Contém apenas um ponto que chamaremos de $u\left(x_{0}\right)$.

Para cada $U^{i}$ escolha uma de suas fronteiras que são determinadas por gráficos de curvas horizontais, a tais curvas escolhidas, chamamos de $u_{i}$, e são gráficos das funções dadas por $y=u_{i}(x)$.

A família $u_{i}$ é uniformemente contínua pois são funções lipschitizianas com constante de lispschitz igual a $\frac{1}{2}$. Além disso tál família é uniformemente limitada por 1. Assim, pelo teorema de Arzelá-Ascoli a seqüência de funções $\left(u_{i}\right)_{i}$ tem subseqüência uniformemente convergente a uma função $u_{\infty}$. Sem perda de generalidade, vamos supor que a própria seqüência é convergente. Como a convergência da família $u_{i}$ é uniforme, $y=u_{x}$ define uma curva horizontal, além disso, para cada $x_{0} \in[0,1]$ temos que 


$$
u_{i}\left(x_{0}\right) \rightarrow u_{\infty}\left(x_{0}\right)
$$

Por outro lado $u_{i}\left(x_{0}\right) \in U^{i}\left(x_{0}\right)$ como $d\left(u^{i}\left(x_{0}\right)\right) \rightarrow 0$ segue que

$$
u_{i}\left(x_{0}\right) \rightarrow u\left(x_{0}\right)
$$

Pela unicidade do limite, para cada $x_{0} \in[0,1]$ temos

$$
u\left(x_{0}\right)=u_{\infty}\left(x_{0}\right)
$$

Assim, as intersecções das faixas horizontais definem uma curva horizontal.

Temos provado o lema, como queríamos.

Lema 1.2. Uma curva horizontal $y=u(x)$ e uma curva vertical $x=v(y)$ intersectam-se em precisamente um ponto.

Demonstração. Sabemos que conseqüência de conexidade as curvas horizontais e verticais se intersectam sempre.

Um ponto na intersecção de tais curvas satisfaz a equação

$$
x=v(u(x))
$$

De fato um ponto $(x, y)$ na intersecção de uma curva horizontal $y=u(x)$ e na curva vertical $x=v(y)$ temos que

$$
\begin{aligned}
& y=u(x) \\
& x=v(y)
\end{aligned}
$$

substituindo o valor de $y$ da primeira na segunda equação obtemos

$$
x=v(u(x))
$$

Suponha então que haja dois pontos $x_{1}$ e $x_{2}$, distintos, na intersecção da curva horizontal $y=u(x)$ com a faixa vertical $x=v(y)$ temos que 


$$
\begin{gathered}
\left|x_{1}-x_{2}-\left(v\left(u\left(x_{1}\right)\right)-v\left(u\left(x_{2}\right)\right)\right)\right|=0 \Rightarrow|| x_{1}-x_{2}|-| v\left(u\left(x_{1}\right)\right)-v\left(u\left(x_{2}\right)\right)||=0 \\
\Rightarrow\left|x_{1}-x_{2}\right|=\left|v\left(u\left(x_{1}\right)\right)-v\left(u\left(x_{2}\right)\right)\right|
\end{gathered}
$$

Por outro lado temos o seguinte

$$
\left|v\left(u\left(x_{1}\right)\right)-v\left(u\left(x_{2}\right)\right)\right| \leq \mu\left|u\left(x_{1}\right)-u\left(x_{2}\right)\right| \leq \mu^{2}\left|x_{1}-x_{2}\right|<\left|x_{1}-x_{2}\right|
$$

o que é uma contradição com

$$
\left|x_{1}-x_{2}\right|=\left|v\left(u\left(x_{1}\right)\right)-v\left(u\left(x_{2}\right)\right)\right|
$$

Fica assim provado o lema.

De acordo com o lema precedente, temos definida uma aplicação que a cada par de curvas (horizontal e vertical), associa em $Q=[0,1] \times[0,1]$ a intersecção das mesmas.

$$
(u, v) \mapsto z=(x, y) \in Q
$$

Essa aplicação é Lipschitz contínua com a norma

$$
\|u\|+\|v\|=\max _{0 \leq x \leq 1}|u(x)|+\max _{0 \leq y \leq 1}|v(y)| ;|z|=|x|+|y|
$$

De fato, seja o ponto $z_{j}$ com $j=1,2$ correspondente a interseção do par de curvas $\left(u_{j}, v_{j}\right) ; j=1,2$

Seja $z_{j}=\left(x_{j}, y_{j}\right)$, sabemos que $x_{j}=v_{j}\left(y_{j}\right)$ e $y_{j}=u_{j}\left(x_{j}\right)$, com isso temos que

$$
\left|x_{1}-x_{2}\right|=\left|v_{1}\left(y_{1}\right)-v_{2}\left(y_{2}\right)\right| \leq\left|v_{1}\left(y_{1}\right)-v_{1}\left(y_{2}\right)\right|+\left|v_{2}\left(y_{2}\right)-v_{1}\left(y_{2}\right)\right| \leq \mu\left|y_{1} y_{2}\right|+|| v_{1}-v_{2}||
$$

analogamente obtemos

$$
\left|y_{1}-y_{2}\right| \leq \mu\left|x_{1}-x_{2}\right|+|| u_{1}-u_{2}||
$$

Somando as equações (1) e (2) temos que 


$$
\begin{gathered}
\left|x_{1}-x_{2}\right|+\left|y_{1}-y_{2}\right| \leq|| u_{1}-u_{2}||+|| v_{1}-v_{2}||+\mu\left(\left|x_{1}-x_{2}\right|+\left|y_{1}-y_{2}\right|\right) \Rightarrow \\
\Rightarrow(1-\mu)\left(\left|x_{1}-x_{2}\right|+\left|y_{1}-y_{2}\right|\right) \leq|| u_{1}-u_{2}||+|| v_{1}-v_{2}|| \Rightarrow \\
\Rightarrow\left|z_{1}-z_{2}\right| \leq(1-\mu)^{-1}\left(|| u_{1}-u_{2}||+|| v_{1}-v_{2}||\right)
\end{gathered}
$$

Isso mostra que a aplicação que a cada par de curvas $(u, v)$, horizontal e vertical respectivamente, que associa o seu ponto de interseção z é uma transformação Lipschitz.

Formularemos agora algumas hipóteses sobre a aplicação $\phi$ definida em $Q$.

i) Seja $A=\{1, \ldots, N\}$. Se $N<+\infty$ ou o conjunto dos números naturais e assumir que $U_{a}$ e $V_{a}$ com $a \in A$ são faixas verticais e horizontais respectivamente, tais que

$$
U_{a} \cap U_{b}=\emptyset \quad \text { se } a \neq b
$$

e

$$
V_{a} \cap V_{b}=\emptyset \quad \text { se } a \neq b .
$$

Assumiremos também que $\phi$ aplica homeomorficamente

$$
\phi\left(V_{a}\right)=U_{a}, \forall a \in A
$$

Além disso vamos requerir que as fronteiras verticais de $V_{a}$ sejam levadas nas fronteiras verticais de $U_{a}$, o mesmo valendo para as fronteiras horizontais.

ii) Se $V$ é uma faixa vertical em $\bigcup_{a \in A} V_{a}$, então para todo $a \in A$ temos

$$
\phi^{-1}(V) \cap V_{a}=\widetilde{V}_{a}
$$

$\widetilde{V}_{a}$ é uma faixa vertical ( não vazia) e para algum $0<\nu<1$ vamos supor que

$$
d\left(\widetilde{V}_{a}\right) \leq \nu d\left(V_{a}\right)
$$


Similarmente, pediremos que a propriedade enunciada acima seja verdadeira para as faixas verticais, trocando-se $V_{a}$ por $U_{a}, V$ por $U$ e $\phi^{-1}$ por $\phi$.

Teorema 1.1 (Conjugação com o Shift ). Se ф é um homeomorfismo satisfazendo i) e ii) com respeito as faixas horizontais $U_{a}$ e as faixas verticais $V_{a}, a \in A$ então este possui o shift $\sigma$, definido sobre o conjunto das seqüências bi-infinitas de elementos de A como um subsistema. Isto é existe um homeomorfismo $\tau: \Sigma_{A} \rightarrow Q$ tal que

$$
\phi \tau=\tau \sigma
$$

Em particular, se $N<\infty, I=\tau\left(\Sigma_{A}\right)$ é um conjunto fechado e invariante em $Q$ e como $\Sigma_{A}$ é um conjunto de Cantor, $I=\Sigma_{A}$ também o é.

Demonstração. Faremos um estudo do conjunto dos pontos $p \in Q$ para os quais $\phi^{-k}(p) \in V_{s_{k}}$, para $(k=0, \pm 1, \pm 2, \ldots)$. Definimos indutivamente

$$
V_{s_{0} s_{-1} \ldots s_{-n}}=V_{s_{0}} \phi^{-1}\left(V_{s_{1} \ldots s_{-n}}\right)
$$

temos que

$$
d\left(V_{s_{0} s_{-1} \ldots s_{-n}}\right) \leq \nu d\left(V_{s_{-1} \ldots s_{-n}}\right) \leq \nu^{2} d\left(V_{s_{-2} \ldots s_{-n}}\right) \leq \ldots \nu^{n} d\left(V_{s_{n}}\right)
$$

Segue disso que

$$
\lim _{n \rightarrow+\infty} d\left(V_{s_{0} \ldots s_{-n}}\right)=0
$$

A partir da nossa definição de $V_{s_{0} \ldots s_{-n}}$ concluímos indutivamente que

$V_{s_{0} \ldots s_{-n}}=\left\{p \in Q \mid \phi^{k}(p) \in V_{s_{-k}}, \quad k=0,1, \ldots n\right\}=\left\{p \in Q \mid \phi^{-k}(p) \in V_{s_{-k}}, \quad k=0,-1, \ldots-n\right\}$

Daí segue que

$$
V_{s_{0} s_{-1} \ldots s_{-n}} \subset V_{s_{0} s_{-1} \ldots s_{-n+1}}
$$

Pelo lema 1.1 definimos a seguinte curva vertical 


$$
v(s)=\bigcap_{n=0}^{+\infty} V_{s_{0} s_{-1} \ldots s_{-n}}=\left\{p \in Q \mid \phi^{-k}(p) \in V_{s_{k}}, k=0,-1 \ldots\right\}
$$

Analogamente, definimos para $n \geq 2$

$$
U_{s_{1} s_{2} \ldots s_{n}}=U_{s_{1}} \cap \phi\left(U_{s_{2} \ldots s_{n}}\right)
$$

e da mesma maneira temos

$$
d\left(U_{s_{1} s_{2} \ldots s_{n}}\right) \nu \leq d\left(U_{s_{2} \ldots s_{n}}\right) \ldots \leq \nu^{n-1} d\left(U_{s_{n}}\right)<\nu^{n-1} \rightarrow 0 \text { quando } n \rightarrow+\infty
$$

Desde que $\phi\left(V_{s_{k}}\right)=U_{s_{k}}$ temos que

$$
U(s)=\bigcap_{n=1}^{+\infty}\left\{p \in Q \mid \phi^{-k+1}(p) \in U_{s_{k}}, k \geq 1\right\}=\left\{p \in Q \mid \phi^{-k}(p) \in V_{s_{k}}, k \geq 1\right\}
$$

De acordo com o lema $1.1 U(s)$ é uma curva horizontal, dependendo do parâmetro $s \in \Sigma_{A}$. Pelo lema $2.1 U(s) \cap V(s)$ é um conjunto não vazio e unitário de modo que

$$
U(s) \cap V(s)=\left\{p \in Q \mid \phi^{-k}(p) \in V_{s_{k}}, k=0, \pm 1, \pm 2, \ldots\right\}
$$

Definimos agora a aplicação $\tau: \Sigma_{A} \rightarrow Q$. A aplicação $\tau$ leva a seqüência $\left(\ldots s_{-1} s_{0} s_{1} s_{2} \ldots\right)$ em $V(s) \cap V(s)$.

A partir desta construção e do lema 1.1 segue que $\tau$ está bem definida. Seja $\sigma: \Sigma_{A} \rightarrow \Sigma_{A}$ tal que

$$
\sigma\left(\left(s_{k}\right)_{k \in \mathbb{Z}}\right)=\left(s_{k-1}\right)_{k \in \mathbb{Z}}
$$

temos que

$$
\tau(\sigma(s))=\phi(p)=\phi \sigma(s)
$$

De fato, pois pela equação (7) temos

$$
\begin{gathered}
U_{\sigma(s)} \cap V_{\sigma(s)}=\left\{p \in Q \mid \phi^{-k}(p) \in V_{s_{k-1}}, k=0, \pm 1, \pm 2, \ldots\right\}= \\
=\left\{p \in Q \mid \phi^{-k-1}(\phi(p)) \in V_{s_{k-1}}, k \in \mathbb{Z}\right\}
\end{gathered}
$$


$\log O$

$$
U_{\sigma(s)} \cap V_{\sigma(s)}=\phi(p=U(s) \cap V(s))
$$

A equação (8) mostra a existência de $\tau: \Sigma_{A} \rightarrow Q$ tal que

$$
\tau \sigma=\phi \tau .
$$

Resta mostra que $\tau$ é uma homeomorfismo. Primeiro provemos a injetividade da aplicação, $\tau$. Sejam $s, t$ duas seqüências distintas em $\Sigma_{A}$. Chame

$$
\begin{aligned}
& p_{s}=U(s) \cap V(s) \\
& p_{t}=U(t) \cap V(t)
\end{aligned}
$$

Se fosse $p_{s}=p_{t}$ então pela como $V_{i} \neq V_{j}, i \neq j$ segue de $(7)$ que $V_{s_{k}}=V_{t_{k}}$ o que implica que $s_{k}=t_{k}$ e disso $s=t$ o que é um absurdo. Logo, a aplicação $\tau$ é injetora.

Mostremos agora a continuidade de $\tau$. Se $s, s^{\prime} \in \Sigma_{A}$ e $s_{i}=s_{i}^{\prime},|i| \leq n$ então isto significa que $\tau(s)$ e $\tau\left(s^{\prime}\right)$ pertencem à mesma faixa horizontal $U_{s_{1} \ldots s_{n}}$ e à mesma faixa vertical $V_{s_{0} \ldots s_{-n}}$ como

$$
d\left(U_{s_{1} \ldots s_{n}}\right) \leq \nu^{n-1}
$$

$\mathrm{e}$

$$
d\left(V_{s_{0} \ldots s_{-n}}\right) \leq \nu^{n}
$$

segue que

$$
\left|\tau(s)-\tau\left(s^{\prime}\right)\right| \leq(1-\mu)^{-1}\left(\nu^{n}+\nu^{n-1}\right) \rightarrow 0 \text { quando } n \rightarrow+\infty
$$

isso mostra a continuidade de $\tau$.

Quando $A$ é finito pelo Teorema de Tychonoff o conjunto $\Sigma_{A}$ é compacto. Como a aplicação $\tau$ é contínua, a sua imagem $\tau\left(\Sigma_{A}\right)$ é contínua.

Sendo $\Sigma_{A}$ um espaço métrico, o mesmo é um espaço Hausdorff além de ser compacto. Como $Q$ também é de Hausdorff, a aplicação $\tau$ é um homeomorfismo de $\Sigma$ com a sua imagem, $\tau\left(\Sigma_{A}\right)$. Como $\Sigma_{A}$ é um conjunto de Cantor, segue que qualquer conjunto homeomorfo a este também o é. Segue disso que $I=\Sigma_{A}$ é um conjunto de Cantor. 
O teorema acima mostra que a dinâmica $\phi$ definido sobre o conjunto $I$ é a mesma, no ponto de vista de conjugação, que a dinâmica do shift sobre $\Sigma_{A}$, conjunto das seqüências bi-infinitas de $N$ símbolos.

Passemos agora ao estudo sobre a estrutura hiperbólica do conjunto invariante $I$.

\subsection{Condições Alternativas para aplicações $\phi$ de Classe $C^{1}$}

Agora nós trocamos a hipótese ii) anterior por

iii) Para algum $\mu \in(0,1)$ definimos um cone, dado que é dado pelo seguinte conjunto de vetores do plano

$$
S^{+}:\left\{(\xi, \eta) \in \mathbb{R}^{2}|| \eta|\leq \mu| \xi \mid\right\}
$$

Futuramente provaremos que $S^{+}$é um cone instável e denotaremos por $C^{u}(\mu)$ onde

$$
C^{u}(\mu)=\left\{(\xi, \eta) \in \mathbb{E}^{u} \times \mathbb{E}^{s}|| \eta|\leq \mu| \xi \mid\right\}
$$

$S^{+}$está definido sobre $\bigcup_{a \in A} U_{a}$, é aplicado nele mesmo pela aplicação $d \phi$, além disso se $\left(\xi_{0}, \eta_{0}\right) \in S^{+}$e $\left(\xi_{1}, \eta_{1}\right)$ é a sua imagem por $d \phi$ então

$$
\left|\xi_{1}\right| \geq \mu^{-1}\left|\xi_{0}\right|
$$

Similarmente, o cone

$$
S^{-}:\left\{(\xi, \eta) \in \mathbb{R}^{2}|| \xi|\leq \mu| \eta \mid\right\}
$$

Futuramente provaremos que $S^{-}$é um cone estável e denotaremos por $C^{s}(\mu)$ onde

$$
C^{s}(\mu)=\left\{(\xi, \eta) \in \mathbb{E}^{u} \times \mathbb{E}^{s}|| \eta|\leq| \xi \mid\right\}
$$

definido sobre $\bigcup_{a \in A} V_{a}$, é aplicado nele mesmo por $d \phi^{-1}$, além disso se $\left(\xi_{1}, \eta_{1}\right)$ é pré-imagem de $\left(\xi_{0}, \eta_{0}\right)$ então 


$$
\left|\eta_{0}\right| \geq \mu^{-1}\left|\eta_{1}\right|
$$

A condição iii) expressa a insatabilidade da aplicação $\phi$ por iterações. Observemos que $d \phi^{n}$ expande a componente horizontal por uma cosntante $\mu^{-n}$, e $d \phi^{-n}$ expande a direção vertical por uma constante $\mu^{-n}$.

Teorema 1.2. Se ф é uma aplicação que é continuamente diferenciável e satisfaz as condições i) e iii) com $\mu \in\left(0, \frac{1}{2}\right)$ então ii) se verifica com $\nu=\mu(1-\mu)^{-1}$ e portanto as afirmações do teorema anterior são verificadas.

Demonstração. É suficiente provar que $i i)$ é conseqüência de $i$ ) e iii). Seja $\gamma$ uma curva vertical na faixa $V_{b}$, com $b \in A$. A curva $\gamma$ intersecta qualquer curva horizontal, em particular, as fronteiras de $U_{a}, a \in A$. Então $\widehat{\gamma}=\gamma \cap U_{a}$ conecta as fronteiras horizontais de $U_{a}$, daí $\phi^{-1}(\widehat{\gamma})$ conecta as fronteiras horizontais de $V_{a}=\phi^{-1}\left(U_{a}\right)$, isto é $y=0$ e $y=1$. Nós queremos mostrar que $\phi^{-1}(\widehat{\gamma})=\phi^{-1}(\gamma) \cap V_{a}$

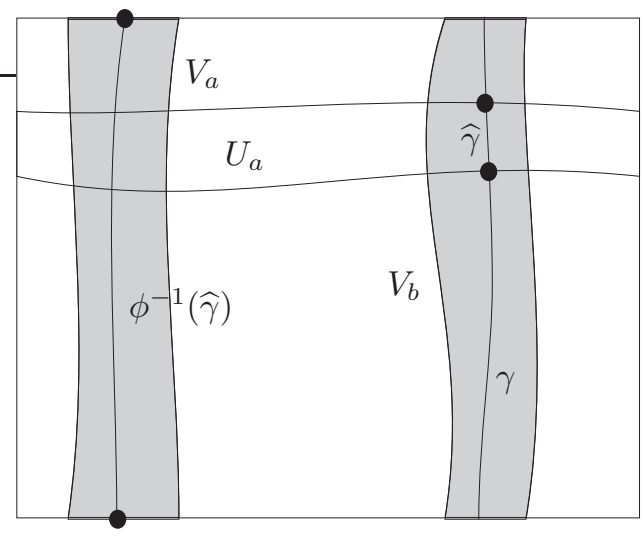

Temos que $\gamma$ é uma curva vertical diferenciável dada por $x=v(y)$, satisfazendo que

$$
\left|v\left(y_{1}\right)-v\left(y_{2}\right)\right| \leq \mu\left|y_{1}-y_{2}\right| \text {. }
$$

Denote por $x_{1}=v\left(y_{1}\right)$ e $x_{2}=v\left(y_{2}\right)$. Seja $\alpha(t), t \in[0,1]$ o segmento que liga os pontos $\left(x_{1}, y_{1}\right)$ e $\left(x_{2}, y_{2}\right)$, como $\gamma$ é uma curva Lipschitz temos que $\alpha^{\prime}(t) \in S^{-}$. 
Sabemos que $d \phi^{-1}$ aplica $S^{-}$em $S^{-}$. Sejam $\left(x_{3}, y_{3}\right),\left(x_{4}, y_{4}\right) \in \phi^{-1}(\widehat{\gamma}) \operatorname{com}\left(x_{3}, y_{3}\right)=$ $\phi^{-1}\left(x_{1}, y_{1}\right)$ e $\left(x_{4}, y_{4}\right)=\phi^{-1}\left(x_{2}, y_{2}\right)$. Osbserve que $\left(x_{3}, y_{3}\right)$ e $\left(x_{4}, y_{4}\right)$ são estremidades da imagem $\phi(\alpha(t))$. Usando a regra da cadeia temos que

$$
D \phi(\alpha(t)) \alpha^{\prime}(t) \in S^{-}
$$

para todo $t \in[0,1]$.

Disso segue que o vetor

$$
\left(x_{4}-x_{3}, y_{4}-y_{3}\right) \in S^{-}
$$

Dessa maneira $\phi^{-1}(\widehat{\gamma})$ é gráfico de uma função $x=w(y)$ com $0 \leq y \leq 1$.

Vamos aplicar essa afirmação às fronteiras de $V \subset V_{b}$ e concluir que a pré-imagem de $\widehat{V}=V \cap U_{a}$ dada por $\phi^{-1}(\widehat{V})=\phi^{-1}(V) \cap V_{a}$ é uma faixa vertical.

Agora falta verificar as informações sobre o diâmetro de $\widehat{V}$ para $0<\mu \leq \frac{1}{2}$ e $r=\frac{\mu}{1-\mu}<1$. Sejam $p_{1}$ e $p_{2}$ dois pontos sobre as fronteiras verticais de $\phi^{-1}(\widehat{V})$ tais que

$$
d\left(\phi^{-1}(\widehat{V})\right)=\left|p_{1}-p_{2}\right|
$$

e que $p_{1}$ e $p_{2}$ tenham a mesma coordenada $y$. Então a curva

$$
p(t)=(1-t) p_{1}+t p_{2}
$$

é paralelo ao eixo dos $x$ e daí $p^{\prime} \in S^{+}$e dessa forma

$$
z(t)=\phi(p(t))
$$

tem tangente

$$
z^{\prime}(t)=d \phi p^{\prime} \in S^{+}
$$

pela hipótese iii). Isso mostra que (da definição de $S^{+}$) que $z(0)$ e $z(1)$ está sobre uma curva horizontal, e cada qual sobre duas linhas verticais que estão a uma distância $d(\widehat{V})$.

Lembremos que

$$
\left|z_{1}-z_{2}\right| \leq(1-\mu)^{-1}\left(|| u_{1}-u_{2}||+|| v_{1}-v_{2}||\right)
$$


Como $z_{0}$ e $z_{1}$ estão sobre a mesma curva horizontal segue da desigualdade acima que

$$
|z(0)-z(1)| \leq(1-\mu)^{-1} \cdot d(\widehat{V})
$$

Finalmente, escrevendo $z(t)=(x(t), y(t))$ temos pela segunda hipótese de $i i i)$ que

$$
\left|x^{\prime}\right| \geq \mu^{-1}\left|p^{\prime}\right|>0
$$

já que $p^{\prime}$ é paralelo ao eixo dos $x$. Com isso segue que $x^{\prime}$ não troca de sinal e

$$
\begin{gathered}
\left|p_{1}-p_{2}\right|=\int_{0}^{1}\left|p^{\prime}\right| d t \mu \int_{0}^{1}\left|x^{\prime}\right| d t \leq \mu|x(1)-x(0)| \leq \\
\leq \mu|z(1)-z(0)| \leq \mu(1-\mu)^{-1} d(\widehat{V}) \leq d(V)
\end{gathered}
$$

Logo, concluímos que

$$
d\left(\phi^{-1}(\widehat{V})\right) \leq \frac{\mu}{1-\mu} d(\widehat{V})
$$

Como queríamos demonstrar.

\subsection{Estrutura Hiperbólica do Conjunto Invariante}

Vamos verficar que o conjunto $I=\tau\left(\Sigma_{A}\right)$ é um conjunto hiperbólico. O que pretendemos é associar a cada ponto $p \in I$ duas linhas retas $L_{p}^{+}$e $L_{p}^{-}$geradas por vetores linearmente independente no espaço tangente a $p$, de forma que $L_{p}^{+}$e $L_{p}^{-}$variem continuamente com $p$ e tais que $L_{p}^{+}$e $L_{p}^{-}$sejam invariantes sobre $\phi$, isto é:

$$
d \phi L_{p}^{ \pm}=L_{\phi(p)}^{ \pm}
$$

e exista $\lambda>1$ e munidos com a norma do máximo tenhamos

$$
\left\{\begin{array}{c}
|d \phi(\xi, \eta)| \geq \lambda|(\xi, \eta)| \text { se }(\xi, \eta) \in L_{p}^{+} \\
\left|d \phi^{-1}(\xi, \eta)\right| \geq \lambda|(\xi, \eta)| \text { se }(\xi, \eta) \in L_{p}^{-}
\end{array}\right.
$$

Seja 


$$
\left(\begin{array}{ll}
f_{x}(p) & f_{y}(p) \\
g_{x}(p) & g_{y}(p)
\end{array}\right)
$$

a matriz jacobiana de $d \phi$ em um ponto $p \in I$. Chame de $\Delta$ o determinante jacobiano $f_{x}(p) g_{y}(p)-$ $f_{y}(p) g_{x}(p)$.

Teorema 1.3. Denotemos por $\Delta$ o determinante Jacobiano de d申 sobre um ponto p. Se para todo ponto $p \in I \Delta, \Delta^{-1} \leq \frac{1}{2} \mu^{-2}$ então I é um conjunto hiperbólico.

Demonstração. Construiremos uma linha $L^{+}$pelo princípio da contração em Espaços de Banach. Para esta proposta, tomemos uma linha qualquer em $S^{+}=S_{p}^{+}$, o cone $S^{+}$com base em $p \in I$. Vamos representar $L^{+}=L_{p}^{+}$na forma

$$
\eta_{0}=\alpha_{p} \xi_{0},\left|\alpha_{p}\right| \leq \mu
$$

Onde $\alpha_{p}$ é uma função contínua de $p \in I$. Por aplicação de $d \phi_{p}$ a $L^{+}$obtemos

$$
\begin{gathered}
\eta_{1}=\alpha_{\phi(p)}^{*} \xi \\
\left(\begin{array}{c}
\xi_{1} \\
\alpha_{\phi(p)}^{*} \xi_{1}
\end{array}\right)=\left(\begin{array}{cc}
f_{x}(p) & f_{y}(p) \\
g_{x}(p) & g_{y}(p)
\end{array}\right)\left(\begin{array}{c}
\xi_{0} \\
\\
\alpha_{\phi(p)}^{*} \xi_{0}
\end{array}\right)
\end{gathered}
$$

Para simplicar ponha

$$
\left(\begin{array}{cc}
f_{x}(p) & f_{y}(p) \\
g_{x}(p) & g_{y}(p)
\end{array}\right)=\left(\begin{array}{cc}
a & b \\
c & d
\end{array}\right)
$$

e com isso segue que

$$
\left(\begin{array}{c}
\xi_{1} \\
\alpha_{\phi(p)}^{*} \xi_{1}
\end{array}\right)=\left(\begin{array}{c}
a \xi+b \alpha_{p} \xi \\
c \xi+d \alpha_{p} \xi
\end{array}\right) \Rightarrow \frac{\alpha_{\phi(p)}^{*} \xi_{1}}{\xi_{1}}=\frac{c \xi_{0}+d \alpha_{p} \xi_{0}}{a \xi_{0}+b \alpha_{p} \xi_{0}} \Rightarrow \alpha_{\phi(p)}^{*}=\frac{c+d \alpha_{p}}{a+b \alpha_{p}}
$$

Defina sobre $p \in I$ a linha $L_{\phi(p)}^{+}$com inclinação $\alpha_{\phi(p)}^{*}$. 
Temos que $d \phi\left(S^{+}\right) \subset S^{+}$segue que $\left|\alpha_{\phi(p)}^{*}\right| \leq \mu$ e por $\left.i i i\right)$ temos que

$$
\left|a+b \alpha_{p}\right| \geq \mu^{-1}
$$

Se $\beta_{p}$ define uma outra linha podemos obter de maneira análoga $\beta_{\phi(p)}^{*}$ e então temos

$$
\begin{gathered}
\left|\alpha_{\phi(p)}^{*}-\beta_{\phi(p)}^{*}\right|=\left|\frac{c+d \alpha_{p}}{a+b \alpha_{p}}-\frac{c+d \beta_{p}}{a+b \beta_{p}}\right|=\frac{a d\left(\alpha_{p}-\beta_{p}\right)+b c\left(\alpha_{p}-\beta_{p}\right)}{\left|a+b \alpha_{p}\right|\left|a+b \beta_{p}\right|}=\frac{\Delta}{\left|a+b \alpha_{p}\right|\left|a+b \beta_{p}\right|}\left|\alpha_{p}-\beta_{p}\right| \leq \\
\leq \frac{1}{2} \frac{\mu^{2}\left|\alpha_{p}-\beta_{p}\right|}{\mu^{-1} \mu^{-1}}=\frac{1}{2}\left|\alpha_{p}-\beta_{p}\right|
\end{gathered}
$$

Logo

$$
\left|\alpha_{\phi(p)}^{*}-\beta_{\phi(p)}^{*}\right| \leq \frac{1}{2}\left|\alpha_{p}-\beta_{p}\right|
$$

Como cada linha em reta $L_{p}$ passando por $p$ com vetor diretor em $S^{+}$é definida pela sua inclinação $\alpha_{p}$, da desigualdade acima, temos que para cada $p \in I$ que a aplicação

$$
\alpha_{p} \mapsto \alpha_{\phi(p)}^{*}
$$

é uma contração num espaço de Banach, assim existe um ponto fixo $c_{p}$ para a aplicação acima.

Como $c_{p}$ é um ponto fixo e $d \phi(p)$ é um isomorfismo, temos que $c_{p}=c_{\phi(p)}^{*}$ e por definição de $c_{\phi(p)}^{*}$ a linha $L_{p}^{+}$de inclinação $c_{p}$ é invariante por $d \phi(p)$

$$
d \phi(p) L_{p}^{+}=L_{\phi(p)}^{+}
$$

Analogamente encontramos uma linha $L_{\phi(p)}^{-}$, invariante por $d \phi^{-1}(p)$ usando as relações análogas

$$
\eta=\alpha_{\phi(p)} \xi,\left|\alpha_{\phi(p)}\right| \leq \mu
$$

e então

$$
\left|\xi_{1}\right|=\left|\widetilde{a}+\widetilde{b} \alpha_{\phi(p)}\right||\xi| \geq \mu^{-1}|\xi|
$$

estamos usando a notação 


$$
d \phi^{-1}(p)=\left(\begin{array}{cc}
\widetilde{a} & \widetilde{b} \\
& \\
\widetilde{c} & \widetilde{d}
\end{array}\right)
$$

Com isso obtemos que

$$
d \phi^{-1}(p) L_{\phi(p)}^{-}=L_{p}^{-} \Rightarrow d \phi(p) L_{p}^{-}=L_{\phi(p)}^{-} .
$$

Tomemos $\lambda=\mu^{-1}$ e temos o provado teorema.

Pode ser provado mais que o teorema anterior. Recordamos que os pontos de $I$ são pontos de intersecção das curvas horizontais $U(s)$ e $V(s)$ determinados respectivamente pelas seqüências $s_{1}, s_{2}, \ldots$ e $s_{0}, s_{-1}, \ldots, s_{-n} \ldots$ Provamos um resultado mais geral.

Teorema 1.4. Sobre as hipóteses i) e iii) com

$$
0<\mu \leq \min \left\{\left(|\Delta|^{1 / 2},|\Delta|^{-1 / 2}\right)\right\}
$$

as curvas $U(s)$ e $V(s)$ são continuamente diferenciáveis e suas tangentes sobre cada ponto $p \in I$ coincide com as linhas $L_{p}^{+}$e $L_{p}^{-}$da estrutura hiperbólica.

Demonstração. É suficiente provar o teorema para as linhas horizontais $U(s)$. Denotamos o conjunto de todas as curvas horizontais por

$$
\mathcal{U}=\bigcup_{s} U(s)
$$

provemos primeiro que

$$
\phi^{-1}(\mathcal{U}) \subset \mathcal{U}
$$

De fato pela definição de $U(s)$ por (5) temos que

$$
U(s)=U_{s_{1}} \cap \phi\left(U_{\sigma(s)}\right)
$$

e daí aplicando $\phi^{-1}$ segue que

$$
\phi^{-1}(\mathcal{U}(s))=V_{s_{1}} \cap U_{\sigma(s)} \subset U_{\sigma(s)}
$$

onde $s=\left(s_{1}, s_{2}, \ldots, s_{n}, \ldots\right)$. isso mostra a afirmação 


$$
U(s)=U_{s_{1}} \cap \phi\left(U_{\sigma(s)}\right)
$$

Escolhemos um ponto $p \in U(s)$, denote por conjunto $T_{p}$ o conjunto de todos os limites das inclinações de linhas secantas passando por dois pontos distintos $q_{1}$ e $q_{2}$ que tendem a $p$ sobre $\operatorname{um} U(s)$.

Vamos supor que $U(s)$ é uma curva horizontal dada por $y=u(x)$. O conjunto $T_{p}$ é o conjunto

de todos os limites possíveis para a razão:

$$
\alpha_{p}=\lim _{n \rightarrow+\infty} \frac{u\left(x_{n}-u\left(x_{n}^{\prime}\right)\right)}{x_{n}-x_{n}^{\prime}}
$$

onde $x_{n}, x_{n}^{\prime} \in[0,1]$ e $x_{n} \neq x_{n}^{\prime}$ e ambas as seqüências tendendo ao mesmo ponto $x_{0}$ tal que $\left(x_{0}, u\left(x_{0}\right)\right)=p \in U(s)$.

Como $y=u(x)$ é $\mu$-lipschitiziana, temos que a razão acima é sempre majorada por $\mu$ e minorada por $-\mu$.

Seja

$$
\omega\left(T_{p}\right)=\max \alpha_{p}-\min \alpha_{p}
$$

Seja $y=\widetilde{u}(x)$, parametrização da curva $\phi(x, u(x)), 0 \leq x \leq 1$. Colocando $\phi(x, y)=$ $(f(x, y), g(x, y))$ onde $f, g: Q \rightarrow \mathbb{R}$ de classe $C^{1}$, temos que

$$
g(x, u(x))=\widetilde{u}(f(x, u(x)))
$$

Aplicando a definição de diferenciabilidade segue que

$$
\begin{gathered}
\widetilde{u}\left(f\left(x_{n}^{\prime}, x_{n}^{\prime}\right)\right)-\widetilde{u}\left(f\left(x_{n}, u\left(x_{n}\right)\right)\right)=g\left(x_{n}^{\prime}, u\left(x_{n}^{\prime}\right)\right)-g\left(x_{n}, u\left(x_{n}\right)\right)=g_{x}\left(x_{n}^{\prime}-x_{n}\right)+g_{y}\left(u\left(x_{n}^{\prime}\right)-u\left(x_{n}\right)\right)+R_{n} \Rightarrow \\
\Rightarrow \frac{\widetilde{u}\left(f\left(x_{n}^{\prime}, u\left(x_{n}^{\prime}\right)\right)\right)-\widetilde{u}\left(f\left(x_{n}, u\left(x_{n}\right)\right)\right)}{x_{n}^{\prime}-x_{n}}=g_{x}+g_{y} \frac{u\left(x_{n}^{\prime}\right)-u\left(x_{n}\right)}{x_{n}^{\prime}-x_{n}}+\frac{R_{n}}{x_{n}^{\prime}-x_{n}}
\end{gathered}
$$

Lembrando que $\left|u\left(x_{n}^{\prime}\right)-u\left(x_{n}\right)\right| \leq \mu\left|x_{n}^{\prime}-x_{n}\right|$ e usando a norma do máximo na desigualdade acima e da definição de diferenciabilidade temos 


$$
\frac{\widetilde{u}\left(f\left(x_{n}^{\prime}, u\left(x_{n}^{\prime}\right)\right)\right)-\widetilde{u}\left(f\left(x_{n}, u\left(x_{n}\right)\right)\right)}{x_{n}^{\prime}-x_{n}}=g_{x}+g_{y} \frac{u\left(x_{n}^{\prime}\right)-u\left(x_{n}\right)}{x_{n}^{\prime}-x_{n}}+\frac{R_{n}}{x_{n}^{\prime}-x_{n}} \rightarrow g_{x}(p) g_{y}(p) \alpha_{p}
$$

Analogamente

$$
f\left(x_{n}^{\prime}, u\left(x_{n}^{\prime}\right)\right)-f\left(x_{n}, u\left(x_{n}\right)\right)
$$

Com isso

$$
\lim _{n \rightarrow+\infty} \frac{\widetilde{u}\left(f\left(x_{n}^{\prime}, u\left(x_{n}^{\prime}\right)\right)\right)-\widetilde{u}\left(f\left(x_{n}, u\left(x_{n}\right)\right)\right)}{\left.f\left(x_{n}^{\prime}, u\left(x_{n}^{\prime}\right)\right)\right)-f\left(x_{n}, u\left(x_{n}\right)\right)}=\frac{g_{x}(p)+g_{y}(p) \alpha_{p}}{f_{x}(p)+f_{y}(p) \alpha_{p}}
$$

Seja $J \phi(p)$ a matriz jacobiana de $\phi$ no ponto $p$. Observemos que

$$
\left(\begin{array}{cc}
f_{x}(p) & f_{y}(p) \\
g_{x}(p) & g_{y}(p)
\end{array}\right)\left(\begin{array}{c}
1 \\
\alpha_{p}
\end{array}\right)=\left(\begin{array}{c}
f_{x}(p)+f_{y}(p) \alpha_{p} \\
g_{x}(p)+g_{y}(p) \alpha_{p}
\end{array}\right) \Rightarrow J \phi(p) T_{p}=T_{p}
$$

Já que $J(\phi(p))$ é um difeomorfismo em cada ponto $p \in \mathcal{U}$.

Sejam $\alpha_{p}$ e $\widetilde{\alpha}_{p}$ obtidas no ponto $p$, note que as hipóteses sobre $\Delta$ implicam as hipóteses sobre $\Delta$ do teorema anterior. Da invariância de $\mathcal{U}$ por $\phi^{-1}$ de modo análogo ao teorema anterior obtemos:

$$
\begin{gathered}
\sup _{p \in \mathcal{U}} \omega\left(T_{p}\right)=\sup _{p \in \mathcal{U}}\left|\widetilde{\alpha}_{p}-\alpha_{p}\right| \leq \frac{1}{2} \sup _{p \in \mathcal{U}}\left|\widetilde{\alpha}_{\phi^{-1}(p)}-\alpha_{\phi^{-1}(p)}\right| \leq \frac{1}{2} \sup _{p \in \mathcal{U}} \omega\left(T_{\phi^{-1}(p)}\right) \leq \frac{1}{2} \sup _{p \in \mathcal{U}} \omega\left(T_{p}\right) \Rightarrow \\
\Rightarrow \sup _{p \in \mathcal{U}} \omega\left(T_{p}\right)=0 \Rightarrow \omega\left(T_{p}\right)=0 \quad \forall p \in \mathcal{U}
\end{gathered}
$$

Assim, da forma em que definimos $T_{p}$ vemos que existe apenas um limite

$$
\lim _{n \rightarrow+\infty} \frac{u\left(x_{n}-u\left(x_{n}^{\prime}\right)\right)}{x_{n}-x_{n}^{\prime}}
$$

para Quaisquer duas seqüências $\left(x_{n}\right)$ e $\left(x_{n}^{\prime}\right)$ dados como anteriormente. Logo, cada curva $y=u(x)$ em $\mathcal{U}$ é diferenciável.

Seja $\Delta_{1}$ a diagonal do quadrado $Q$.

Para provar que tais curvas $U(s)$ gráficos de curvas lipschitizianas $y=u(x)$, são continuamente diferenciáveis, basta notar que 


$$
f: Q \backslash \Delta_{1} \rightarrow[-\mu, \mu]
$$

tal que

$$
f(x, y)=\frac{u(x)-u(y)}{x-y}
$$

é uma função contínua, a qual se extende continuamente aos pontos de acumulação em $\Delta_{1}$.

Na prova do teorema anterior, observemos que o espaço $L_{p}^{ \pm}$são obtidos como limites de espaços secantes pelo ponto $p$. Assim, como $U(s)$ e $V(s)$ são curvas diferenciáveis em $p$, concluímos da construção feita anteriormente que $T_{p} U(s)=L_{p}^{+}$e $T_{p} V(s)=L_{p}^{-}$. Como queríamos demonstrar. 


\section{Estudo Qualitativo e Probabilístico do Problema dos Três Corpos}

\subsection{Formulação do Problema}

Nós vamos aplicar os resultados estudados no capítulo anterior para realizar o estudo do problema dos três corpos restrito. A configuração estudada é devida a Sitnikov, que é a seguinte: Consideraremos dois corpos de massa $m_{1}=m_{2}=\frac{1}{2}$, chamados corpos primários. Esses corpos move-se num em órbitas elípticas em torno de um centro de massa $O$. Queremos descrever o todos os tipos de movimento de um terceiro corpo de massa $m_{3} \approx 0$ de modo a não interferir no movimento dos corpos primários. Este corpo de massa $m_{3}$ movimenta-se sobre a reta $L$, passando por $O$ e é ortogonal ao plano de movimento dos corpos primários.

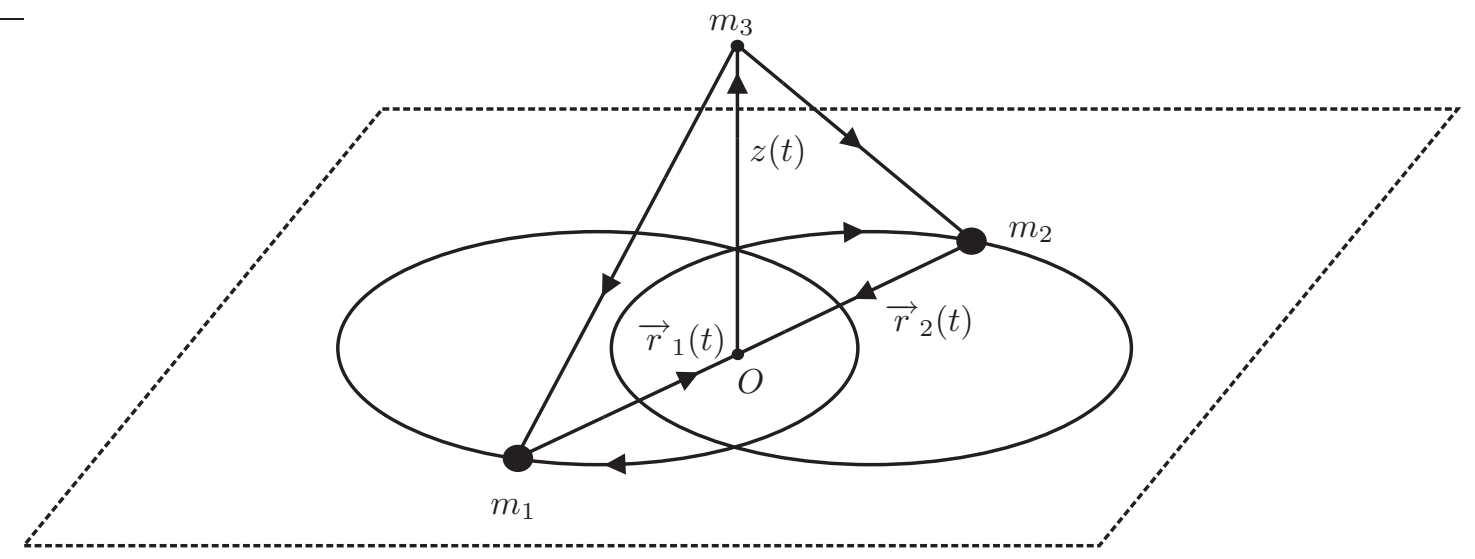

Figura 4: Configuração do Problema

Também estamos normalizando o período da órbita dos corpos primário, como $2 \pi$ e por simplicidade a consideramos como a unidade, a constante de gravitação. Desde que $m_{1}=m_{2}$, as distâncias de cada um dos corpos primários a $O$ é a mesma, e chamamos de $r(t)$. Aqui $r$ é uma função real analítica e periódica de período $2 \pi$.

Estamos assumindo que

$$
r(t)=1-\varepsilon \cos t+O\left(\varepsilon^{2}\right)
$$


Assim, pela simetria do problema a equação diferencial que governa o movimento de $m_{3}$ é

$$
\ddot{z}=-\frac{z}{\left(z^{2}+r(t)^{2}\right)}
$$

De fato, coloquemos

$$
\left\|\vec{r}_{1}(t)\right\|=\left\|\vec{r}_{2}(t)\right\|=r(t)
$$

como acima. Observe que

$$
\vec{r}_{1}(t)=-\vec{r}_{2}(t)
$$

Defina os seguintes vetores

$$
\begin{aligned}
& v_{1}(t)=\vec{r}_{1}(t)-z(t) \\
& v_{2}(t)=\vec{r}_{2}(t)-z(t)
\end{aligned}
$$

Pela lei de Newton temos:

$$
\begin{gathered}
m_{3} \ddot{z}=\frac{1}{2} m_{3} \frac{v_{1}(t)}{\left\|v_{1}(t)\right\|}+\frac{1}{2} m_{3} \frac{v_{2}(t)}{\left\|v_{2}(t)\right\|}=\frac{1}{2} \frac{\vec{r}_{1}(t)-z(t)}{\left\|v_{1}(t)\right\|^{3}}+\frac{1}{2} \frac{\vec{r}_{2}(t)-z(t)}{\left\|v_{2}(t)\right\|^{3}}=-\frac{1}{2} \frac{2 z}{\left(z^{2}+r(t)\right)^{3 / 2}} m_{3} \Rightarrow \\
\ddot{z}=-\frac{z}{\left(z^{2}+r(t)^{2}\right)}
\end{gathered}
$$

Analisamos o problema com condições iniciais

$$
z\left(t_{0}\right)=0, \dot{z}\left(t_{0}\right)=v_{0}>0
$$

Basta analisar o caso $v_{0}>0$, podemos ver que se $z(t)$ é solução de (13) então $\widetilde{z}(t)=-z(t)$ também o é com condições inciciais

$$
\widetilde{z}(t)=0, \dot{\widetilde{z}}\left(t_{0}\right)=-v_{0}
$$

A menos de sinal o comportamento de $z(t)$ e $\widetilde{z}(t)$ é exatamente o mesmo.

Observamos que $z(t) \equiv 0$ é a solução com condições iniciais 


$$
z\left(t_{0}\right)=0, v_{0}=0
$$

Também uma solução qualquer de (13) tem pelo menos um zero, que corresponde a passagem pelo ponto $O$. De fato, suponha sem perda de generalidade que $z(t)>0$ para todo $t$, assim, por (13) temos que $z(t)$ é positiva para todo $t$ e côncava para baixo o que não pode ocorrer, já que o gráfico de $z$ fica abaixo do gráfico de qualquer de suas retas tangentes.

Vamos tratar o problema sob um ponto de vista mais geral, fazendo um estudo sobre as seguintes equações de segunda ordem

$$
\ddot{z}=-Q(z, t)
$$

Observamos que equação (14) descreve vários fenômenos físicos associados a oscilações periódicas além do problema restrito dos três corpos segundo Sitnikov, descrita por (13).

\subsection{Propriedades Gerais das Soluções da Equação $\ddot{z}=-Q(z, t)$}

Consideramos a seguinte equação diferencial (14) de forma que a $Q$ satisfaça as seguintes condições:

$1^{\circ} Q(z, t)$ é de classe $C^{1}$ em $\mathbb{R}^{2}$.

$2^{\circ} Q(z, t)$ é impar em $z$ e $2 \pi$ periódica em $t$.

$3^{\circ} Q(z, t)>0$ para todo $x>0$ e para todo $t \in \mathbb{R}$.

$4^{\circ}$ Existe uma função integrável $\psi(z)$ integrável em $[0,+\infty)$ tal que

$$
\left|\frac{\partial Q}{\partial t}(z, t)\right| \leq \psi(z)
$$

Definição 2.1. Uma solução $z(t)$ de (13) é dita hiperbólica, para $t \rightarrow+\infty$ quando

$$
\lim _{t \rightarrow+\infty} \dot{z}(t)=v_{\infty} \neq 0, \lim _{t \rightarrow+\infty} z(t)=\infty
$$

é dito parabólica para $t \rightarrow+\infty$ se

$$
\lim _{t \rightarrow+\infty} \dot{z}(t)=0, \lim _{t \rightarrow+\infty} z(t)=\infty
$$


e é oscilatória para $t \rightarrow+\infty$ quando exite uma seqüência de tempos $\left(t_{n}\right)$ tal que $\lim _{n \rightarrow+\infty}\left(t_{n}\right)=$ $+\infty$ tal que $z\left(t_{n}\right)=0$ para todo $n \in \mathbb{N}$.

A mesma definição pode ser feita para $t \rightarrow-\infty$ Com isso temos:

Proposição 2.1. Cada solução $z(t)$ de (13) para $t \rightarrow+\infty(t \rightarrow-\infty)$ ou é identicamente nula, parabólica, hiperbólica ou oscilatória.

Demonstração. Observamos que como $Q(z, t)$ é ímpar em z temos que

$$
Q(0, t) \equiv 0
$$

logo a solução identicamente nula é solução de (14).

Seja $z(t)$ uma solução com infinitos zeros no semi-eixo real positivo, se $t_{0}$ é um desses zeros temos que

$$
z\left(t_{0}\right)=0, \dot{z}\left(t_{0}\right) \neq 0
$$

pois se fosse ocorre $\dot{z}\left(t_{0}\right)=0$, pelo teorema de existência e unicidade de soluções de equações diferenciais ordinárias, teríamos $z \equiv 0$. O que não ocorre.

Por outro lado

$$
z\left(t_{0}\right)=0, \dot{z}\left(t_{0}\right) \neq 0
$$

mostra que cada zero de $z(t)$ é isolado, logo, os zeros de $z(t)$ podem estar contidos num conjunto limitado, se isso ocorre tais zeros se aculariam em $\tilde{t}$ que por continuidade das soluções seria um zero não isolado de $z(t)$. O que não pode ocorrer. A mesma demonstração pode ser feita de maneira análoga para o caso $z(t)$ com infinitos zeros no eixo real negativo.

Agora consideremos o caso em que $z(t)$ tem um número finito de zeros no semi-eixo real positivo. Seja $t_{0}$ o máximo destes zeros. temos

$$
z\left(t_{0}\right)=0, \dot{z}\left(t_{0}\right) \neq 0
$$

Suponha sem perda de generalidade que $\dot{z}\left(t_{0}\right)>0$ daí para $t>t_{0}$, temos que $z(t)>0$ e pela equação (14) a função $\dot{z}$ é decrescente para $t>t_{0}$ daí existe o limite 


$$
\lim _{t \rightarrow+\infty} \dot{z}(t) \geq 0
$$

resta mostra que se

$$
\lim _{t \rightarrow+\infty} \dot{z}(t)=0
$$

tenhamos também

$$
\lim _{t \rightarrow+\infty} z(t)=+\infty
$$

Sabemos que $\dot{z}(t) \geq 0$ e $z(t)$ é não decrescente. Suponha que $\lim _{t \rightarrow+\infty} \leq b<+\infty$.

Integrando (14) obtemos

$$
\int_{t_{0}}^{+\infty} Q(z(s), s) d s=\lim _{t \rightarrow+\infty} \int_{t_{0}}^{t} Q(z(s), s) d s=\lim _{t \rightarrow+\infty}\left(\dot{z}\left(t_{0}\right)-\dot{z}(t)\right)=\dot{z}\left(t_{0}\right)
$$

Dessa forma, a integral acima converge e pelo critério de Cauchy

$$
\lim _{n \rightarrow+\infty} \int_{2 n \pi}^{2(n+1) \pi} Q(z(s), s) d s=0
$$

daí

$$
0=\lim _{n \rightarrow+\infty} \int_{2 n \pi}^{2(n+1) \pi} Q(z(s), s) d s=\lim _{n \rightarrow+\infty} \int_{0}^{2 \pi} Q(z(s+2 \pi n), s) d s=\int_{0}^{2 \pi} Q(b, s)
$$

Desde que $z(s) \leq z(s+2 \pi n) \leq b$ e $z(s+2 \pi n) \rightarrow b$ uniformente.

Então $\int_{0}^{2 \pi} Q(z(s+2 \pi n), s) d s=0$ contradiz $3^{\circ}$. o que completa a demonstração da proposição.

Essa é a primeira informação que temos sobre o comportamento no infinito das soluções de (13). Todo nosso estudo concentra-se em analisar qualitativamente o comportamento das soluções de (13) no infinito.

Seja $z(t, v, \tau)$ uma solução determinada pelas condições iniciais

$$
z(\tau, v, \tau)=0, \dot{z}(\tau, v, \tau)=v
$$

Como $Q(z, t)$ é $2 \pi$ periódico em $t$ e ímpar em $z$, nós temos que 


$$
\begin{gathered}
z(t, v, \tau) \equiv z(t+2 \pi, v, \tau+2 \pi) \\
z(t,-v, \tau) \equiv-z(t, v, \tau)
\end{gathered}
$$

As indentidade (16) e (17), nos dizem que podemos trabalhar apenas com velocidades positivas, em nossas condições inicias, e $\tau(\bmod 2 \pi)$

Tais identidades decorrem imediatamente do teorema de existência e unicidade de equações diferenciais ordinárias. Seja $v>0$ e considere $\left(\tau, \tau^{\prime}\right)$ um intervalo maximal onde $z(t, v, \tau)$ é positiva. Pode ocorrer $\tau^{\prime}=+\infty$. Introduziremos agora a seguinte notação

$$
Z^{+}(v, \tau)=\sup _{\tau<t<\tau^{\prime}} z(t, v, \tau) .
$$

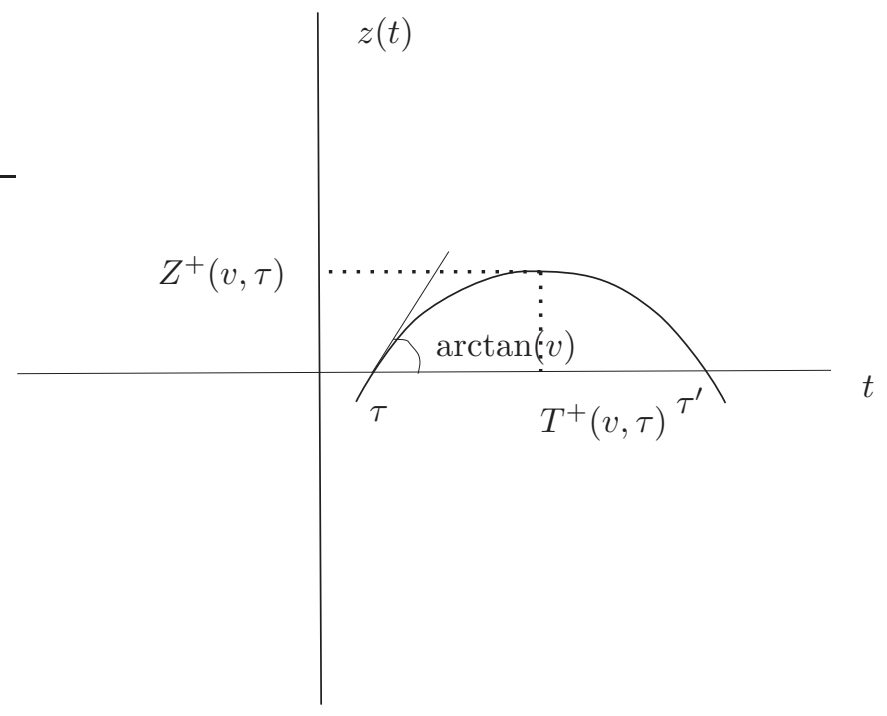

Se $\tau^{\prime}=+\infty$, então a solução $z(t)$ é parabólica e daí $Z(v, \tau)=+\infty$. Se $\tau^{\prime}$ é finito, temos que $z\left(\tau^{\prime}\right)=0$ e com isso

$$
Z^{+}(v, \tau)=\max _{\tau<t<\tau^{\prime}} z(t, v, \tau)
$$

Ao tempo $t$ em que $z(t, v, \tau)$ atinge o ponto $Z^{+}(v, \tau)$, chamamos de $T^{+}(v, \tau)$. Como estamos supondo que $Q(z, t)>0$ para $z>0$ o tempo $T^{+}(v, \tau)$ é determinado unicamente. De fato, se $Z^{+}(v, \tau)=+\infty$, estamos no caso em que $z(t)$ é não decrescente para $t>\tau$, é parabólica ou 
hiperbólica, daí colocamos $T^{+}(v, \tau)=+\infty$. Se $Z^{+}(v, \tau)$ é finito, então $\tau^{\prime}<\infty$ e daí sabemos que em $\left(\tau, \tau^{\prime}\right), \ddot{z}<0$, então $z(t)$ é côncava para baixo e $\dot{z}$ é decrescente, com isso $z$ atinge uma única vez o seu máximo $Z^{+}(v, \tau)$.

Após atingir o seu máximo, a solução $z(t)$ decresce até $z\left(\tau, v, \tau^{\prime}\right)=0$ com isso temos

$$
\begin{array}{llll}
z(t, v, \tau)<Z(v, \tau) & \dot{z}(t, v, \tau)>0 & \text { se } & \tau<t<T^{+}(v, \tau) \\
z(t, v, \tau)=Z(v, \tau) & \dot{z}(t, v, \tau)=0 & \text { se } & t=T^{+}(v, \tau) \\
z(t, v, \tau)<Z(v, \tau) & \dot{z}(t, v, \tau)<0 & \text { se } & T^{+}(v, \tau)<t<\tau^{\prime}
\end{array}
$$

Definimos $Z^{+}(0, \tau) \equiv 0$

A primeira preocupação com as funções $Z^{+}$e $T^{+}$é com respeito à continuidade em suas variáveis.

\section{Proposição 2.2.}

1) A função $Z^{+}$é contínua para todo $\tau$ e $v \geq 0$ e $T^{+}$é contínua para todo $\tau$ e $v>0$.

2) Se $\left(\tau_{n}\right)$ é uma seqüência limitada inferiormente, $Q(z, t)$ é limitada superiormente, $\left(v_{n}\right)$ uma seqüência de velocidades positivas tal que $v_{n} \rightarrow+\infty$ e $Z\left(v_{n}, \tau_{n}\right) \rightarrow+\infty$ então $\tau_{n}^{\prime} \rightarrow+\infty$ e conseqüentemene $T^{+}\left(\tau_{n}, v_{n}\right) \rightarrow+\infty$

Demonstração. Primeiramente provemos a parte 1) da proposição.

Consideremos inicialmente o caso em que $v_{0}>0$ e $\tau^{\prime}<+\infty$. Selecionamos $t_{1}$ e $t_{2}$ tal que

$$
\tau<t_{1}<T^{+}\left(v_{0}, \tau_{0}\right)<t_{2}<\tau^{\prime}
$$

pelas equações (18) sabemos que, $\dot{z}\left(t, v_{0}, \tau_{0}\right)>0$ para $\tau<t<t_{1}$ e $\dot{z}\left(t_{2}, v_{0}, \tau_{0}\right)<0$. Para um $\varepsilon>0$ suficientemente pequeno o ponto $\left(v_{0}, \tau_{0}\right)$ tem uma vizinhança pequena, $U_{0}$ tal que:

$$
\begin{gathered}
\dot{z}(t, v, \tau)>0 \text { para } \tau \leq t \leq t_{1} \\
\dot{z}\left(t_{2}, v, \tau\right)<0 \text { e }\left|z(t, v, \tau)-z\left(t, v_{0}, \tau_{0}\right)\right|<\varepsilon \text { se } t_{1} \leq t \leq t_{2}
\end{gathered}
$$

as equações acima são conseqüências do teorema de depemdência contína e teorema da função implícita, visto que colocamos $z(\tau, v, \tau)=0$ e $\dot{z}(\tau, v, \tau)=v \neq 0$, localmente $z$ é uma função de $\tau$, isto nos permite, localmente, substituir as condições iniciais $\left(v_{0}, z_{0}\right)$ por $\left(v_{0}, \tau_{0}\right)$.

A partir das equações (19) e (20) temos que 


$$
t_{1}<T^{+}(v, \tau)<t_{2}
$$

Observe que, como $t_{1}$ e $t_{2}$ são escolhidos arbitrariamente pequenos, dado $\varepsilon>0$ tão pequeno podemos encontrar uma vizinhança $U_{0}$ de $\left(v_{0}, \tau_{0}\right)$ tal que aconteça

$$
t_{1}<T^{+}(v, \tau)<t_{2}
$$

Por este processo concluímos que

$$
\lim _{(v, \tau) \rightarrow\left(v_{0}, \tau_{0}\right)} T^{+}(v, \tau)=T^{+}\left(v_{0}, \tau_{0}\right) .
$$

Isso prova a continuidade de $T^{+}$no caso em que $v_{0}>0$.

Usando a desigualdade triangular, podemos estimar a quantidade

$$
\left|Z^{+}(t, v, \tau)-Z^{+}\left(t_{0}, v_{0}, \tau_{0}\right)\right|
$$

por

$$
\left|Z^{+}(t, v, \tau)-z\left(t_{1}, v, \tau\right)\right|+\left|z\left(t_{1}, v, \tau\right)-z\left(t_{1}, v_{0}, \tau_{0}\right)\right|+\left|Z^{+}\left(t_{0}, v_{0}, \tau_{0}\right)-z\left(t_{1}, v_{0}, \tau_{0}\right)\right|
$$

A partir de (20) e continuidade uniforme, podemos fazer com que cada parcela da soma acima seja menor que $\varepsilon$.

E com isso vemos que $Z^{+}$é contínua no caso em que $v_{0}>0$.

Até agora tratamos do caso em que $\tau^{\prime}<+\infty$. No caso em que $\tau^{\prime}=+\infty$ temos que

$$
Z^{+}\left(v_{0}, \tau_{0}\right)=+\infty=T^{+}\left(v_{0}, \tau_{0}\right)
$$

Usando teorema de dependência contínua das equações diferenciais ordinárias, temos que para qualquer $\varepsilon>0$ e intervalo compacto $\left[\tau, t_{0}\right] \subset[\tau,+\infty]$, se $(v, \tau)$ está suficientemente próximo de $\left(v_{0}, \tau_{0}\right)$ temos:

$$
\left|z\left(t, v_{0}, \tau_{0}\right)-z(t, v, \tau)\right|<\varepsilon
$$

para todo $t \in\left[\tau, t_{0}\right]$. Isso prova que $T^{+}$e $Z^{+}$são contínuas no caso em que $\tau^{\prime}=+\infty$. 
Para concluir a prova da parte 1) da proposição, ainda resta prová-la no caso em que $v_{0}=0$.

Seguiremos então a prova por contradição. Supondo que $Z^{+}(v, \tau)$ não é contínua em $v=0$, existe uma seqüência $\left(v_{n}, \tau_{n}\right)$ convergindo a $\left(0, \tau_{0}\right)$ tal que $Z^{+}\left(v_{n}, \tau_{n}\right) \rightarrow \alpha>0$. Não estamos dscartando a possibilidade de termos $\alpha=+\infty$.

Se $\alpha$ for finito, então, do teorema de dependência contínua, as trajetórias $z\left(t, v_{n}, \tau_{n}\right)$ devem ficar arbitrariamente próximas de $z\left(t, 0, \tau_{0}\right) \equiv 0$ para tempos arbitrariamente grandes, obrigando $Z^{+}\left(v_{n}, \tau_{n}\right)$ convergir a zero, o que é uma contradição com $Z^{+}\left(v_{n}, \tau_{n}\right)$ convergir a $\alpha>0$.

Se $\alpha=+\infty$, desde que

$$
Z^{+}(v, \tau)=\sup _{\left(\tau, \tau^{\prime}\right)} z(t, v, \tau)=\sup _{\left(\tau, T^{+}(v, \tau)\right)} z(t, v, \tau)
$$

pela própria definição de supremo, podemos escolher uma seqüência de tempos $t_{n}$ tal que

$$
z\left(t_{n}, v_{n}, \tau_{n}\right) \rightarrow \alpha^{\prime}<\alpha
$$

Observe que podemos supor que $\alpha^{\prime}$ é finito.

Sobre o intervalo $\left[\tau_{n}, t_{n}\right]$ a derivada $\dot{z}\left(t, v_{n}, \tau_{n}\right)$ é não crescente, pelas equações $(18)$, e dessa forma temos

$$
0 \leq \dot{z}\left(t, v_{n}, \tau_{n}\right) \leq v_{n}
$$

Com isso, segue que:

$$
z\left(t_{n}, v_{n}, \tau_{n}\right)=\int_{\tau_{n}}^{t_{n}} \dot{z}\left(t, v_{n}, \tau_{n}\right) d t \leq v_{n}\left(t_{n}-\tau_{n}\right)
$$

de maneira que

$$
t_{n}-\tau_{n} \geq \frac{z\left(t_{n}, v_{n}, \tau_{n}\right)}{v_{n}} \rightarrow+\infty
$$

pois já sabemos que $v_{n} \rightarrow 0$ e $\alpha^{\prime}$ é finito. Sendo assim, podemos escolher os $t_{n}$ de forma que $t_{n}-2 \pi>\tau_{n}$.

Além disso temos que

$$
\dot{z}\left(t_{n}, v_{n}, \tau_{n}\right)=v_{n}-\int_{\tau_{n}}^{t_{n}} Q\left(z\left(t, v_{n}, \tau_{n}\right), t\right) d t \geq 0
$$


e então

$$
0 \leq \int_{t_{n}-2 \pi}^{t_{n}} Q\left(z\left(t, v_{n}, \tau_{n}\right), t\right) \leq \int_{\tau_{n}}^{t_{n}} Q\left(z\left(t, v_{n}, \tau_{n}\right), t\right) d t \leq v_{n} \rightarrow 0
$$

Conseqüentemente o limite

$$
\lim _{n \rightarrow+\infty} \int_{t_{n}-2 \pi}^{t_{n}} Q\left(\left(z\left(t, v_{n}, \tau_{n}\right), t\right)\right) d t=0
$$

Desde que $0 \leq \dot{z}\left(t, v_{n}, \tau_{n}\right) \leq v_{n}$, pelo teorema do valor médio, a oscilação de $z\left(t, v_{n}, \tau_{n}\right)$ em $\left[t_{n}-2 \pi, t_{n}\right]$, não excede a $2 \pi v_{n}$.

$$
\left|z\left(t, v_{n}, \tau_{n}\right)-z\left(\bar{t}, v_{n}, \tau_{n}\right)\right| \leq 2 \pi v_{n}
$$

quaisquer que sejam $t, \bar{t} \in\left[t_{n}-2 \pi, t_{n}\right]$.

com isso a seqüência de funções $z\left(t_{n}-2 \pi+s, \tau_{n}\right)$ com $s \in[0,2 \pi]$ converge uniformemente à função constante $\alpha^{\prime}$.

De fato

$$
\left|z\left(t_{n}, v_{n}, \tau_{n}\right)-z\left(t_{n}-2 \pi+s, \tau_{n}\right)\right| \leq 2 \pi v_{n}
$$

sabemos que $z\left(t_{n}, v_{n}, \tau_{n}\right) \rightarrow \alpha^{\prime}$, usando a continuidade do módulo e fazendo $n \rightarrow+\infty$ na desiguadade acima obtemos a convergência uniforme

$$
z\left(t_{n}-2 \pi+s, v_{n}, \tau_{n}\right) \rightarrow \alpha^{\prime}
$$

Nas integrais

$$
\int_{t_{n}-2 \pi}^{t_{n}} Q\left(\left(z\left(t, v_{n}, \tau_{n}\right), t\right)\right) d t
$$

façamos a seguinte mudança de variáveis:

$$
u=t-t_{n}+2 \pi
$$

assim essas integrais ganham a forma

$$
\int_{0}^{2 \pi} Q\left(z\left(u+t_{n}-2 \pi, v_{n}, \tau_{n}\right), u+t_{n}+2 \pi\right) d u
$$


como $Q(z, t)$ é $2 \pi-$ periódica em $t$ segue que

$\int_{0}^{2 \pi} Q\left(z\left(u+t_{n}-2 \pi, v_{n}, \tau_{n}\right), u+t_{n}+2 \pi\right)=\int_{0}^{2 \pi} Q\left(z\left(u+t_{n}-2 \pi, v_{n}, \tau_{n}\right), u+t_{n}(\bmod 2 \pi)\right) d u$

Observe que $t_{n}(\bmod 2 \pi)$ é limitada e tem uma subseqüência convergente em $[0,2 \pi]$. Assumiremos que a própria seqüência $t_{n}(\bmod 2 \pi)$ convirja a $t_{0} \in[0,2 \pi]$.

Assim, por (21) segue que

$$
\begin{gathered}
0=\lim _{n \rightarrow+\infty} \int_{0}^{2 \pi} Q\left(z\left(u+t_{n}-2 \pi, v_{n}, \tau_{n}\right), u+t_{n}(\bmod 2 \pi)\right) d u= \\
\int_{0}^{2 \pi} \lim _{n \rightarrow+\infty} Q\left(z\left(u+t_{n}-2 \pi, v_{n}, \tau_{n}\right), u+t_{n}(\bmod 2 \pi)\right) d t=\int_{0}^{2 \pi} Q\left(\alpha^{\prime}, u+t_{0}\right) d u .
\end{gathered}
$$

Como, $\alpha^{\prime}>0$ e $Q\left(\alpha^{\prime}, u+t_{0}\right)>0, \forall t \in[0,2 \pi]$ a integral

$$
\int_{0}^{2 \pi} Q\left(\alpha^{\prime}, u+t_{0}\right) d u>0
$$

o que é uma contradição com (22). Assim $Z^{+}(v, \tau)$ é contínua em $v=0$.

Com isso, encerramos a parte 1) da proposição.

Passemos agora à prova da segunda parte desta proposição. Prosseguiremos por contradição. Suponha que, nas hipóteses dadas em 2) exista uma seqüência $\left(v_{n}, \tau_{n}\right)$ com

$$
Z^{+}\left(v_{n}, \tau_{n}\right) \rightarrow+\infty, \quad \tau_{n} \geq 0>-\infty, \quad T^{+}\left(v_{n}, \tau_{n}\right) \rightarrow b<+\infty .
$$

Estamos considerando, sem perda de generalidade $\tau(\bmod 2 \pi)$. A menos de passarmos a uma subseqüência, também, sem perda de generalidade suporemos que

$$
v_{n} \rightarrow v_{0} \tau_{n} \rightarrow \tau_{0} \in[0,2 \pi]
$$

Observe que $v_{0}$ pode ser infinito. Se for $v_{0}<+\infty$ temos que

$$
Z^{+}\left(v_{0}, \tau_{0}\right)=\lim _{n \rightarrow+\infty} Z^{+}\left(v_{n}, \tau_{n}\right)+\infty
$$

pela continuidade de $Z^{+}$. e com isso obtemos que $z\left(t, v_{0}, \tau_{0}\right)$ é parabólico ou hiperbólico, em ambos os casos $T^{+}\left(v_{0}, \tau_{0}\right)=+\infty$. O que é um absurdo já que $T^{+}\left(v_{n}, \tau_{n}\right) \rightarrow b<+\infty$. 
Note que neste caso $v_{0} \neq 0$, caso contrário teríamos $Z^{+}\left(v_{0}, \tau_{0}\right)=0$.

Se for $v_{0}=+\infty$ temos que

$$
\begin{gathered}
\int_{\tau_{n}}^{T^{+}\left(v_{n}, \tau_{n}\right)} Q\left(z\left(s, v_{n}, \tau_{n}\right), s\right) d s=-\int_{\tau_{n}}^{T^{+}\left(v_{n}, \tau_{n}\right)} \ddot{z}\left(s, v_{n}, \tau_{n}\right) d s= \\
=\dot{z}\left(\tau_{n}, v_{n}, \tau_{n}\right)-\dot{z}\left(T^{+}\left(v_{n}, \tau_{n}\right)\right)=v_{n} \rightarrow+\infty
\end{gathered}
$$

Por outro lado, estamos supondo que $Q(z, t)$ é limitado por uma constante $C$ para qualquer $(z, t) \in \mathbb{R}^{2}$ daí

$$
\int_{\tau_{n}}^{T^{+}\left(v_{n}, \tau_{n}\right)} Q\left(z\left(s, v_{n}, \tau_{n}\right), s\right) d s \leq C\left(T^{+}\left(v_{n}, \tau_{n}\right)-\tau_{n}\right) \rightarrow C\left(b-\tau_{0}\right)<+\infty .
$$

Mais isso é uma contradição com a igualdade acima.

Logo a parte 2) desta proposição é verdadeira, e concluímos a nossa demonstração.

A parte 2) da proposição acima, pode ser interpretada da seguinte forma: que sob as hipóteses de 2), $T^{+}$cresce monotonamente com $Z^{+}$tendendo ao infinito, quando $Z^{+}$cresce infinitamente.

Introduziremos agora a seguinte notação

$$
Q_{0}(z)=\frac{1}{2 \pi} \int_{0}^{2 \pi} Q(z, t) d t
$$

Observe que $Q_{0}$ é a média sobre $t$ em $Q(z, t)$. A partir de agora veremos que as soluções da equação diferencial (14), apresentam o mesmo comportamento qualitativo da seguinte equação média

$$
\ddot{z}=-Q_{0}(z)
$$

No sentido de que $Q_{0}$ trará boas informações sobre comportamento no infinito da equação (14).

Lema 2.1. Se $4^{\circ}$ é satisfeita para todo $x>0$ e para todo t então

$$
\left|Q_{0}(z)-Q(z)\right| \leq 2 \pi \psi(z)
$$

Demonstração. Sabemo que

$$
\left|Q_{0}(z)-Q(z)\right|=\left|\frac{1}{2 \pi} \int_{0}^{2 \pi} Q(z, t) d t-Q(z, t)\right|=\left|\frac{1}{2 \pi} Q\left(z, t_{z}\right) \cdot 2 \pi-Q(z, t)\right|
$$


onde $t_{z} \in[0,2 \pi]$ daí usando o teorema do valor médio e $4^{\circ}$ temos

$$
\left|Q\left(z, t_{z}\right)-Q(z, t)\right|=\left|\frac{\partial Q}{\partial t}\left(z, t_{z}^{\prime}\right)\right|\left|t_{z}^{\prime}-t\right| \leq 2 \pi \psi(z)
$$

Introduziremos agora a seguinte notação

$$
h^{+}(v, \tau)=\left\{\begin{array}{cc}
\frac{v_{\infty}^{2}}{2}+\int_{0}^{+\infty} Q_{0}(z) d z & \text { se } Z^{+}(v, \tau)=+\infty \\
\int_{0}^{Z^{+}(v, \tau)} Q_{0}(z) d z & \text { se } Z^{+}(v, \tau)<+\infty
\end{array}\right.
$$

Chamaremos $\Im=\int_{0}^{+\infty} Q_{0}(z) d z$. Com relação a $h^{+}$temos No caso autônomo,temos que a fórmula (23) coincide com

$$
h(t)=\frac{z \dot{(t)}^{2}}{2}+\int_{0}^{z(t)} Q(y) d y
$$

Quando (14) é autônomo, isto é, $Q(z, t)=Q(z)$ não depende de $t$, a função $h^{+}$representa a energia total.

Observamos que, por aplicação do teorema fundamental do cálculo e regra da cadeia $h(t)$ é constante, de fato:

$$
h^{\prime}(t)=\dot{z}(t) \ddot{z}(t)+Q(z(t)) \dot{z}(t)=\dot{z}(t) \ddot{z}(t)-\dot{z}(t) \ddot{z}(t)=0
$$

já que $z(t)$ satisfaz a equação diferencial (14) com $Q(z, t)=Q(z)$.

Proposição 2.3. A função $h^{+}(v, \tau)$ é contínua para todo $\tau$ e $v \geq 0$ e satisfaz

$$
\frac{v^{2}}{2}-2 \pi \int_{0}^{+\infty} \psi(z) d z \leq h^{+}(v, \tau) \leq \frac{v^{2}}{2}+2 \pi \int_{0}^{+\infty} \psi(z) d z
$$

Além disso, se existirem funções reais $Q(z)$ e $q(z)$ tais que

$$
0 \leq q(z) \leq Q(z, t) \leq Q(z)
$$

então

$$
\frac{v^{2}}{2}-\int_{0}^{Z^{+}(v, \tau)} Q(z) d z \leq h^{+}(v, \tau)-\int_{0}^{Z^{+}(v, \tau)} Q_{0}(z) d z \leq \frac{v^{2}}{2}-\int_{0}^{Z^{+}(v, \tau)} q(z) d z
$$


Demonstração. No intervalo $\left[\tau, T^{+}(v, \tau)\right]$ a solução de $(14), z(t, v, \tau)$ é monótona crescente em $t$, já que $\dot{z}(t, v, \tau)>0$. Dessa forma, a equação

$$
z(t, v, \tau)=y
$$

determina uma solução $t(y, v, \tau) \in\left[\tau, T^{+}(v, \tau)\right]$ tal que $z(t(y, v, \tau), v, \tau)=y$. Tomando-se a expressão

$$
f(t, y, v, \tau)=z(t, v, \tau)-y
$$

e a derivando com relação a variável $t$ obtemos

$$
\frac{d f}{d t}=\dot{z}(t, v, \tau) \neq 0, \quad \forall t \in\left[\tau, T^{+}(v, \tau)\right)
$$

logo pelo teorema da função implícita, para as soluções $t(y, v, \tau)$ é uma função diferenciável em suas entradas, desde $y \in\left[0, Z^{+}(v, \tau)\right)$. Além disso, $t(y, v, \tau)$ é contínua em todas as suas entradas quando $y \in\left[0, Z^{+}(v, \tau)\right]$.

Queremos calcular a derivada de $t(y, v, \tau)$ com relação a $y$ quando este percorre o intervalo $\left[0, Z^{+}(v, \tau)\right)$

$$
z(t, v, \tau)-y=0 \Rightarrow \frac{\partial z}{\partial t} \frac{\partial t}{\partial y}-1=0 \Rightarrow \frac{\partial t}{\partial y}=\left(\frac{\partial z}{\partial t}\right)^{-1}=\frac{1}{\dot{z}(t(y, v, \tau), v, \tau)}
$$

Temos que $\dot{z}(t(y, v, \tau), v, \tau)$ é também contínua na região $0 \leq y \leq Z^{+}(v, \tau)$ e anula-se quando $y=Z^{+}(v, \tau)$, essa função decresce de $v$ a 0 , sendo positiva para

$$
0 \leq y \leq Z^{+}(v, \tau)
$$

Seja

$$
V(y, v, \tau)=\left\{\begin{array}{cl}
\dot{z}(t(y, v, \tau), v, \tau) & ; \quad 0 \leq y \leq Z^{+}(v, \tau) \\
0 & ; \quad Z^{+}(v, \tau) \leq y<+\infty
\end{array}\right.
$$

A continuidade de $V(y, v, \tau)$ é verficada, quando checamos a validade da fórmula (27) no ponto $y=Z^{+}(v, \tau)$.

Seja $y_{0}=Z^{+}\left(v_{0}, \tau_{0}\right)<+\infty$ e $\varepsilon>0$ arbitrário. Desde que $\dot{z}\left(t\left(y, v_{0}, \tau_{0}\right)\right)$ decresce até 0 quando $y$ cresce até $y_{0}$. Nós podemos escolher $y_{1}<y_{0}$ tal que 


$$
0<\dot{z}\left(t\left(y_{1}, v_{0}, \tau_{0}\right), v_{0}, \tau_{0}\right)<\varepsilon
$$

Por continuidade, existe uma vizinhança $U_{0}$ de $\left(v_{0}, \tau_{0}\right)$, tal que para todo $(v, \tau) \in U_{0}$ tem-se que

$$
0<\dot{z}\left(t\left(y_{1}, v, \tau\right), v, \tau\right)<\varepsilon, \quad y_{1}<Z^{+}(v, \tau)
$$

O conjunto $\left\{(y, v, \tau) \mid y>y_{1}, \quad(v, \tau) \in U_{0}\right\}$ é uma vizinhança de $\left(y_{0}, v_{0}, \tau_{0}\right)$ e por $(27)$ e $(28)$ temos que $|V(y, v, \tau)-0|=V(y, v, \tau)<\varepsilon$ nessa vizinhança. Já que $V$ é não crescente em $y$, dessa maneira $V(y, v, \tau)$ é contínua na superfície $y=Z^{+}(v, \tau)$ e portanto, em todo o seu domínio.

Para $0 \leq y<Z^{+}(v, \tau), V$ está bem definida e é diferenciável em seus argumentos.

Por (14) e (27) temos que

$$
\frac{\partial V}{\partial y}=\frac{\partial}{\partial y}[\dot{z}(t(y, v, \tau), v, \tau)]=\ddot{z}(t(y, v, \tau)) \frac{\partial t}{\partial y}=-Q(y, t(y, v, \tau)) \frac{1}{V(y, v, \tau)}
$$

Definimos agora a seguinte função

$$
h^{+}(y, v, \tau)=\left\{\begin{array}{cc}
\frac{V(y, v, \tau)^{2}}{2}+\int_{0}^{+\infty} Q_{0}(z) d z & \text { se } 0 \leq y<Z^{+}(v, \tau) \\
\int_{0}^{Z^{+}(v, \tau)} Q_{0}(\xi) d \xi & \text { se } Z^{+}(v, \tau) \leq y \leq+\infty
\end{array}\right.
$$

Essa função é contínua, já que ambas as expressões que a define são contínuas em seus respectivos domínios e coincidem sobre a superfície $y=Z^{+}(v, \tau)$. Além do mais, em cada uma das regiões onde $h^{+}(y, v, \tau)$ está definida, esta é diferenciável.

Derivando $h^{+}(y, v, \tau)$ em cada uma das regiões de sua definição obtemos:

$$
\frac{\partial h^{+}}{\partial y}(y, v, \tau)=\left\{\begin{array}{cc}
-\frac{2 V}{2} Q \frac{1}{V}+Q_{0}(y), & 0 \leq y<Z^{+}(v, \tau) \\
0, & Z^{+}(v, \tau)<y \leq+\infty
\end{array}\right.
$$

que se reduz a 


$$
\frac{\partial h^{+}}{\partial y}(y, v, \tau)=\left\{\begin{array}{cc}
Q_{0}(y)-Q(y, t(y, v, \tau)), & 0 \leq y<Z^{+}(v, \tau) \\
0, & Z^{+}(v, \tau)<y \leq+\infty
\end{array}\right.
$$

Sobre a superfície $y=Z^{+}(v, \tau)$, as expressões dadas em (32) não necessariamente coincidem.

Pelo lema 2.1, a derivada $\frac{\partial h}{\partial y}(y, v, \tau)$ pode ser majorada por $2 \pi \phi(z)$, a qual é integrável no intervalo $[0+\infty]$. Assim, existe o limite

$$
\lim _{y \rightarrow+\infty} h^{+}(y, v, \tau)=h^{+}(0, v, \tau)+\int_{0}^{+\infty} \frac{\partial h^{+}}{\partial y}(y, v, \tau) d y=\frac{v}{2}^{2}+\int_{0}^{Z^{+}(v, \tau)}\left[Q_{0}(y)-Q(y, t)\right] d y
$$

Tal convergência depende apenas de $y$ logo, a mesma é uniforme em $(v, \tau)$. Mas para $Z^{+}(v, \tau)=+\infty$, a solução correspondente $z(t, v, \tau)$ é parabólica ou hiperbólica e isso faz com que

$$
\lim _{y \rightarrow+\infty} V(y, v, \tau)=v_{\infty}
$$

fazendo em (30) $y \rightarrow+\infty$ o que obtemos é a equação (23). Com isso, temos que

$$
h^{+}(v, \tau)=\lim _{y \rightarrow+\infty} h^{+}(y, v, \tau)=\frac{v^{2}}{2}+\int_{0}^{Z^{+}(v, \tau)}\left[Q_{0}(y)-Q(y, t(y, v, \tau))\right] d y
$$

Da equação (33) temos que

$$
\left|h^{+}(v, \tau)-\frac{v^{2}}{2}\right| \leq \int_{0}^{+\infty}\left|Q_{0}(y)-Q(y, t(y, v, \tau))\right| d y \leq 2 \pi \int_{0}^{+\infty} \phi(z) d z
$$

daí então

$$
\frac{v}{2}^{2}-2 \pi \int_{0}^{+\infty} \phi(z) d z \leq h^{+}(v, \tau) \leq \frac{v}{2}^{2}+2 \pi \int_{0}^{+\infty} \phi(z) d z
$$

Se (24) é satisfeita segue de (33) que

$$
\frac{v^{2}}{2}+\int_{0}^{Z^{+}(v, \tau)}\left[Q_{0}(y)-Q(y)\right] d y \leq h^{+}(v, \tau) \leq \frac{v^{2}}{2}+\int_{0}^{Z^{+}(v, \tau)}\left[Q_{0}(y)-q(y)\right] d y
$$

Disso segue que (25) e isso termina a prova desta proposição.

Para facilitar nossos argumentos a partir de agora faremos uso da simetria do problema. Primeiramente observamos que a partir de (16) as funções $h^{+}, Z^{+}$e $T^{+}$são periódicas em $\tau$ 
com período $2 \pi$. Assim é natural considerar $\tau$ como coordenada angular e considerar somente $v \geq 0$, como falamos anteriormente. E como $Z(0, \tau) \equiv 0, h^{+}(0, \tau) \equiv 0$ podemos identificar o ponto $(0, \tau)$ como a origem de um plano $\Phi$ descrito pelas coordenadas polares $(v, \tau)$.

Veremos que as características das soluções de (14) dependem $\Im$ ser ou não finito. Dividimos $\Phi$ nos seguintes subconjuntos disjuntos

$$
\begin{gathered}
\{O\}=\{v \equiv 0\} \\
R_{0}^{+}=\left\{(v, \tau) \mid 0<h^{+}(v, \tau)<\Im\right\} \\
\Pi_{0}^{+}=\left\{(v, \tau) \mid 0<h^{+}(v, \tau)=\Im\right\} \\
H_{0}^{+}=\left\{(v, \tau) \mid 0<h^{+}(v, \tau)<\Im\right\}
\end{gathered}
$$

Teorema 2.1. O conjunto $R_{0}^{+}$é aberto e não vazio e se $(v, \tau) \in R_{0}^{+}$a solução associada $z(t, v, \tau)$ retorna a $\Phi$ pelo menos uma vez para $t>\tau$. Se $\Im=+\infty$, entãa toda solução nãa trivial de (14) é oscilatória. Se $\Im<+\infty$, então $\Pi_{0}^{+}$é não vazio e a solução z $(t, v, \tau)$ é parabólica para $(v, \tau) \in \Pi_{0}^{+}$e a solução $z(t, v, \tau)$ é hiperbólica para $(v, \tau) \in H_{0}^{+}$.

Demonstração. Se $\Im$ é finito então a continuidade de $h^{+}$garante que os conjuntos $R_{0}^{+}$e $H_{0}^{+}$ são abertos e $\Pi_{0}^{+}$é fechado. Observe que $h^{+}$é contínua e satisfaz

$$
\frac{v^{2}}{2}-2 \pi \int_{0}^{+\infty} \psi(z) d z \leq h^{+}(v, \tau) \leq \frac{v^{2}}{2}+2 \pi \int_{0}^{+\infty} \psi(z) d z
$$

Sendo assim para qualquer ponto do plano $\Phi, h^{+}$é finita, pois $\psi$ é integrável em $[0,+\infty)$. A desigualdade acima, mostra que $h^{+}$assume valores arbitrariamente grandes. Como $h^{+}(0, \tau)=0$ então por continuidade de $h^{+}$o conjunto $R_{0}^{+}$é não vazio. Além disso se $(v, \tau) \in R_{0}^{+}$então, pela definição de $h^{+}$, temos que $Z^{+}(v, \tau)$ é finito, daí a mesma retorna ao plano $\Phi$.

Se $\Im=+\infty$, como $h^{+}$é sempre finita, $Z^{+}(v, \tau)$ é finito para qualquer $(v, \tau)$. Neste caso qualquer trajetória $z(t, v, \tau)$ é oscilatória. Caso, contrário existiria $\left(v^{\prime}, \tau^{\prime}\right)$, tal que $Z^{+}\left(v^{\prime}, \tau^{\prime}\right)=$ $+\infty$ e com isso $h^{+}\left(v^{\prime}, \tau^{\prime}\right)$ seria infinito.

Para um ponto $(v, \tau) \in H_{0}^{+}$, por ser $Q_{0}(z)>0$ para $z>0$ temos que $Z^{+}(v, \tau)=\infty$, caso contrário seria $h^{+}(v, \tau)<\Im$, o que contradiz a definição de $H_{0}^{+}$. Sendo $(v, \tau) \in H_{0}^{+}$devemos ter $v_{\infty}>0 . \operatorname{Logo} z(t, v, \tau)$ é hiperbólica.

Por disjunção dos conjuntos envolvidos no teorema, resta apenas que: se $(v, \tau) \in \Pi_{0}^{+}$então $z(t, v, \tau)$ é parabólica 
Munidos do teorema precedente e das fórmulas (24) e (25) temos

Corolário 2.1. $O$ conjunto $\{O\} \cup R_{0}^{+}$está contido num disco de raio $\left[2 \Im+4 \pi \int_{0}^{+\infty} \psi(z) d z\right]^{1 / 2}$. Além disso valendo (24) podemos melhorar a estimativa acima, concluindo que $\{O\} \cup R_{0}^{+}$está contido num disco de raio $\left[2 \int_{0}^{+\infty} Q(z) d z\right]^{1 / 2}$, e o mesmo contém um disco de raio $\left[2 \int_{0}^{+\infty} q(z) d z\right]^{1 / 2}$.

Demonstração. Começamos observando que se $\Im=+\infty$ este corolário é satisfeito trivialmente, então vamos nos concentrar no caso $\Im$ finito.

Em $R_{0}^{+} \cup\{O\}$ temos que $h^{+}(v, \tau) \leq \Im$ e juntamente (34) segue que

$$
\frac{v^{2}}{2}-2 \pi \int_{0}^{+\infty} \phi(z) d z \leq h^{+}(v, \tau) \leq \Im
$$

com isso

$$
\frac{v^{2}}{2}-2 \pi \int_{0}^{+\infty} \phi(z) d z \leq \Im \Rightarrow|v| \leq\left[2 \Im+4 \pi \int_{0}^{+\infty} \psi(z) d z\right]^{1 / 2}
$$

Assim $R_{0}^{+} \cup\{O\}$ está contido num disco de raio $\left[2 \Im+4 \pi \int_{0}^{+\infty} \psi(z) d z\right]^{1 / 2}$.

Em (24), como $Q(z)$ e $q(z)$ são independentes de $t$, fazendo a média temporal para se obter $Q_{0}(z)$, vemos que (24) é satisfeita, trocando-se $Q(z, t)$ por $Q_{0}(z)$.

Se as desigualdades (24) são satisfeitas segue de (25) que

$$
\frac{v^{2}}{2}+\int_{0}^{Z^{+}(v, \tau)} Q_{0}(z) d z \leq h^{+}(v, \tau)+\int_{0}^{Z^{+}(v, \tau)} Q(z) d z
$$

e

$$
h^{+}(v, \tau)+\int_{0}^{Z^{+}(v, \tau)} q(z) d z \leq \frac{v^{2}}{2}+\int_{0}^{Z^{+}(v, \tau)} Q_{0}(z) d z
$$

Em ambas as desigualdades acima, vem de $(24)$ que $Z^{+}(v, \tau)$ pode ser substituído por $+\infty$. Assim, obtemos

$$
\frac{v^{2}}{2}-\int_{0}^{+\infty} Q(z) d z \leq h^{+}(v, \tau)-\Im \leq \frac{v^{2}}{2}-\int_{0}^{+\infty} q(z) d z
$$

Agora se $(v, \tau) \in R_{0}^{+} \cup\{O\}$ então $h^{+}(v, \tau)-\Im<0$ e daí se o lado esquerdo de (24) é satisfeita temos que 


$$
\frac{v^{2}}{2}-\int_{0}^{+\infty} Q(z) d z \leq h^{+}(v, \tau)-\Im \leq 0 \Rightarrow \frac{v^{2}}{2}-\int_{0}^{+\infty} Q(z) d z \leq 0
$$

isolando $|v|$ convenientemente vemos que $R_{0}^{+} \cup\{O\}$ está contido num disco de raio $\left[2 \int_{0}^{+\infty} q(z) d z\right]^{1 / 2}$. E se o lado esquerdo de (24) é satisfeita isolando $|v|$ convenientemente em

$$
0 \leq \frac{v^{2}}{2}-\int_{0}^{+\infty} q(z) d z
$$

concluímos a demonstração deste corolário.

A partir do momento em que provarmos que $H_{0}^{+}, \Pi_{0}^{+}, R_{0}^{+}$são conjuntos mensuráveis de $\Phi$, o corolário acima diz que a medida de Lebesgue de $R_{0}^{+}$é positiva.

Tudo que foi elaborado até pode ser feito também para tempos $t<\tau$. Dessa forma podemos definir $Z^{-}\left(v^{\prime}, \tau^{\prime}\right)$ como sendo o valor absoluto do ínfimo de $-z(t, v, \tau)$, no intervalo $\left(\tau, \tau^{\prime}\right)$, onde $z(t, v, \tau)$ é positiva. Procedendo de maneira análoga se $\tau^{\prime}$ é finito ou não. Então de maneira análoga definimos funções $Z^{-}, T^{-}$e $h^{-}$. E tudo que foi provado para as funções $Z^{+}, T^{+}$e $h^{+}$ continuam válidos para $Z^{-}, T^{-}$e $h^{-}$, de modo análogo.

Definição 2.2. Seja $S: R_{0}^{+} \longrightarrow R_{0}^{-}$de forma que $S$ aplica o par $(v, \tau)$ em $\left(v^{\prime}, \tau^{\prime}\right)$ de modo que

$$
z\left(\tau^{\prime}, v, \tau\right)=0, \dot{z}\left(\tau^{\prime}, v, \tau\right)+v^{\prime}=0
$$

Observemos que $S(v, \tau)$ é injetora pelo teorema de existência e unicidade de equações diferenciais ordinárias. Além disso $S$ é sobre, pois para casa $\left(v^{\prime}, \tau^{\prime}\right) \in R_{0}^{-}$a solução $z$ correspondente tem um zero $\tau$ para $\left(t<\tau^{\prime}\right)$, com velocidade de módulo $-v$, assim $S(-v, \tau)=\left(v^{\prime}, \tau^{\prime}\right)$

Proposição 2.4. As seguintes identidades são verificadas

$$
Z^{-} \circ S=Z^{+}, h^{-} \circ S=h^{+}, \quad T^{-} \circ S=T^{+} .
$$

A demostração desta proposição segue diretamente das definições das funções envolvidas. 


\subsection{Estudo Probabilístico do Problema dos Três Corpos}

No estudo probabilístico de Sistemas Dinâmicos, desempenham papel fundamental as transformações que preservam medidas de probabilidade, campo onde podemos aplicar o Teorema de Recorrência de Poincaré.

Teorema 2.2 (Recorrência de Poincaré). Seja $X$ um espaço métrico separável e $T: X \longrightarrow$ X uma aplicação mensurável. Seja $\mu$ uma medida de probabilidade em $X$, invariante sob a aplicação T. Então $\mu(\{x \mid x \notin \omega(x)\})=0$. Ou seja, quase todo ponto $x \in X$ é recorrente.

Proposição 2.5. A aplicação $S: R_{0}^{+} \longrightarrow R_{0}^{-}$é um difeomorfismo que preserva o elemento de área vdvdt em $\Phi$. Se definirmos $S(O)=O$, então a aplicação $S: R_{0}^{+} \cup\{O\} \longrightarrow R_{0}^{-} \cup\{O\}$ é um homeomorfimo que preserva vdvdt.

Observe que a medida $\mu=v d v d t$ em $\Phi$ é absolutamente contínua com respeito à medida de Lebesgue em $\mathbb{R}^{2}$.

Demonstração. Fixemos o par $(v, \tau)$ e consideremos a seguinte aplicação

$$
\left(v^{\prime}, \tau^{\prime}\right) \mapsto\left(z\left(\tau^{\prime}, v, \tau\right), \dot{z}\left(\tau^{\prime}, v, \tau\right)+v^{\prime}\right)
$$

Calculando o Jacobiano da aplicação acima num ponto $\left(v^{\prime}, \tau^{\prime}\right)=S(v, \tau)$ obtemos

$$
\frac{\partial\left(z, \dot{z}+v^{\prime}\right)}{\partial\left(v^{\prime}, \tau^{\prime}\right)}=\left|\begin{array}{cc}
0 & \dot{z}\left(\tau^{\prime}, v, \tau\right) \\
1 & \ddot{z}\left(\tau^{\prime}, v, \tau\right)
\end{array}\right|=-\dot{z}\left(\tau^{\prime}, v, \tau\right)=-v^{\prime} \neq 0
$$

Assim, pelo teorema da função implícita $\left(v^{\prime}, \tau^{\prime}\right)$ depende diferenciávelmente de $(v, \tau)$, pela unicidade do teorema da função implicita $\left(v^{\prime}, \tau^{\prime}\right)=S(v, \tau)$ é diferenciável em $(v, \tau)$.

Por outro lado, fixados $\left(v^{\prime}, \tau^{\prime}\right)$, ao calcularmos o jacobiano $J(v, \tau)$ da aplicação

$$
(v, \tau) \mapsto\left(z\left(\tau, v^{\prime}, \tau^{\prime}\right), \dot{z}\left(\tau, v^{\prime}, \tau^{\prime}\right)+v\right)
$$

Concluímos que

$$
J(v, \tau) \neq 0
$$

daí a inversa $S^{-1}$ de $S$ também é difernciável. Logo $S$ é um dífeo local e como $S$ é bijetora, concluímos que $S$ é um difeomorfismo global, exceto na origem. 
A equação diferencial (14) é equivalente ao sistema Hamiltoniano canônico abaixo.

$$
\left\{\begin{array}{l}
\dot{z}=\frac{\partial H}{\partial v} \\
\dot{v}=-\frac{\partial H}{\partial z}
\end{array} \quad, \quad H(z, v)=\frac{v^{2}}{2}+\int_{0}^{z} Q(y, t) d y\right.
$$

Teorema 2.3 (Poincaré-Cartan). Seja o seguinte Sistema Hamiltoniano

$$
\left\{\begin{array}{l}
\dot{p}=-\frac{\partial H}{\partial q} \\
\dot{q}=\frac{\partial H}{\partial p}
\end{array}\right.
$$

Suponha que $\gamma_{1}$ e $\gamma_{2}$ são duas curvas envolvendo o mesmo tubo de trajetórias do hamiltoniano acima. Então a integral da forma pdq-Hdt é a mesma ao longo de $\gamma_{1}$ e $\gamma_{2}$.

$$
\oint_{\gamma_{1}} p d q-H d q=\oint_{\gamma_{2}} p d q-H d q
$$

Usando a teoria de Integrais Invariantes de Poincaré-Cartan, temos que a 1-forma diferencial

$$
v d z-H d t
$$

é preservado pelo fluxo, que restrita ao plano $z=0$ é a aplicação $S$. Em $z \equiv 0$ temos $d z=0$ e daí a forma de Poincaré-Cartan se reduz a $-\frac{v^{2}}{2} d t$, cuja derivada é elemento de área

$$
-v d v \wedge d t
$$

Assim, por aplicação do teorema de Stokes. A aplicação $S$ preserva o elemento de área $v d v d t$.

Resta agora provar que $S: R_{0}^{+} \cup\{O\} \rightarrow R_{0}^{-} \cup\{O\}$ é um homeomorfismo. Provamos que $Z^{+}$é contínua em $\{O\}$, anulando-se somente neste ponto. O mesmo valendo para o dual $Z^{-}$. Seja $\left(v_{n}, \tau_{n}\right)$ convergindo ao ponto $O$, daí temos que $\left(v_{n}\right)$ converge a zero. Pela proposições 4.4 e 4.2 temos que

$$
Z^{+}\left(v_{n}, \tau_{n}\right)=Z^{-} \circ S\left(v_{n}, \tau_{n}\right)
$$

converge a $O, \operatorname{logo} S\left(v_{n}, \tau_{n}\right)$ converge a $O$ quando $\left(v_{n}, \tau_{n}\right)$ converge a $O$. Com isso $S$ é contínua na origem, e de maneira análoga $S^{-1}$ também o é. Assim $S: R_{0}^{+} \cup\{O\} \longrightarrow R_{0}^{-} \cup\{O\}$ é um homeomorfismo que preserva o elemento de área $v d v d t$. 
Nós consideremos a possibilidade de iterar $S$ repetidas vezes, claro que só poderos fazer isso para pontos $(v, \tau) \in R_{0}^{+}$. Nas demais regiões, a solução correspondente não retorna ao plano $z=0$. Se $(v, \tau) \in S^{-1}\left(H_{0}^{+} \cap R_{0}^{-}\right)$então a solução correspondente retorna uma vez ao plano $\Phi$ para $t>\tau$ depois escapa hiperbolicamente para infinito.

Definamos recursivamente, para $n \geq 1$

$$
\begin{array}{ll}
H_{n}^{+}=S^{-1}\left(H_{n-1}^{+} \cap R_{0}^{-}\right), & H^{+}=\bigcup_{n=0}^{+\infty} H_{n}^{+} \\
\Pi_{n}^{+}=S^{-1}\left(\Pi_{n-1}^{+} \cap R_{0}^{-}\right), & \Pi^{+}=\bigcup_{n=0}^{+\infty} \Pi_{n}^{+} \\
R_{n}^{+}=S^{-1}\left(R_{n-1}^{+} \cap R_{0}^{-}\right), & H^{+}=\bigcap_{n=0}^{+\infty} R_{n}^{+}
\end{array}
$$

1) $H_{n}^{+}$é o conjunto dos $(v, \tau)$ cuja solução correspondente retorna exatamente $n$ vezes ao plano $\Phi$ para $t>\tau$, depois tende hiperbolicamente para infinito.

2) $\Pi_{n}^{+}$é o conjunto dos $(v, \tau)$ cuja solução correspondente retorna exatamente $n$ vezes ao plano $\Phi$ para $t>\tau$, depois tende parabolicamente para infinito.

3) $R_{n}^{+}$é o conjunto dos $(v, \tau)$ cuja solução correspondente retorna pelo menos $n+1$ vezes ao plano $\Phi$ para $t>\tau$.

De maneira análoga podemos definir os conjuntos em (37) com o para os conjuntos $H_{n}^{-}, \Pi_{n}^{-}$ e $R_{n}^{-}$para aplicação $S^{-1}$, e suas descrições são as mesmas que as dos conjuntos definidos em (37) para $t<\tau$. 
Proposição 2.6. Para todo $n \geq 1$ temos

$$
\begin{aligned}
& R_{n+1}^{+}=R_{n}^{+} \cup \Pi_{n}^{+} \cup H_{n}^{+} \\
& R_{n+1}^{-}=R_{n}^{-} \cup \Pi_{n}^{-} \cup H_{n}^{-}
\end{aligned}
$$

A solução $z(t, v, \tau)$ é hiperpólica, parabólica ou oscilatória para $t \rightarrow+\infty(t \rightarrow-\infty)$, se e somente se $(v, \tau) \in H^{+}, \Pi^{+}$ou $R^{+}\left((v, \tau) \in H^{-}, \Pi^{-}\right.$ou $\left.R^{-}\right)$respectivamente.

Além disso

$$
\Phi \backslash\{O\}=H^{+} \cup \Pi^{+} \cup R^{+}=H^{-} \cup \Pi^{-} \cup R^{-}
$$

Os conjuntos $H_{n}^{+}, H^{+}, H_{n}^{-}, H^{-}, R_{n}^{+}, R_{n}^{-}$são abertos, $\Pi_{0}^{+} \cup \ldots \Pi_{n}^{+}$e $\Pi_{0}^{-} \cup \ldots \Pi_{n}^{-}$são fechados; $\Pi^{+}$e $\Pi^{-}$são do tipo $F_{\sigma}$ e os conjuntos $R^{+}$e $R^{-}$são do tipo $G_{\delta}$.

Demonstração. A primeira equação em (38) é equivalente a

$$
S\left(R_{n-1}^{+}\right)=S\left(R_{n}^{+} \cup \Pi_{n}^{+} \cup H_{n}^{+}\right)=S\left(R_{n}^{+}\right) \cup S\left(\Pi_{n}^{+}\right) \cup S\left(H_{n}^{+}\right)=\left(R_{n-1}^{+} \cup \Pi_{n-1}^{+} \cup H_{n-1}^{+}\right) \cap R_{0}^{-} .
$$

Para $n=1$ a indentidade resume-se a $S\left(R_{0}^{+}\right)=R_{0}^{-}$

Para $n>1$ prosseguiremos por indução finita sobre $n$. Suponha, que a proposição seja válida para $1,2, \ldots, n-1$

$$
\begin{gathered}
R_{n}^{+}=S^{-1}\left(R_{n-1}^{+} \cap R_{0}^{-}\right)=S^{-1}\left(\left(R_{n}^{+} \cup \Pi_{n}^{+} \cup H_{n}^{+}\right) \cap R_{0}^{-}\right) \Rightarrow \\
S\left(R_{n}^{+}\right)=\left(R_{n}^{+} \cup \Pi_{n}^{+} \cup H_{n}^{+}\right) \cap R_{0}^{-}
\end{gathered}
$$

e isso completa a indução. Logo a primeira identidade em (38) é verdadeira. Analogamente, prova-se a segunda das identidades.

Se em $\Phi \backslash\{O\}=R_{0}^{+} \cup \Pi_{0}^{+} \cup H_{0}^{+}$trocarmos $R_{0}$ por $R_{1}^{+} \cup \Pi_{1}^{+} \cup H_{1}^{+}$obtemos

$$
\Phi \backslash\{O\}=R_{1}^{+} \cup \Pi_{0}^{+} \cup H_{0}^{+} \cup H_{1}^{+}
$$


onde $R_{1}^{+} \subset R_{0}+$, logo $R_{1}^{+}=R_{0}^{+} \cap R_{1}^{+}$. De modo mais geral temos que $R_{n}^{+} \subset R_{n+1}^{+}, n \in \mathbb{N}$. Assim, de maneira geral temos que:

$$
\Phi \backslash\{O\}=R_{n+1}^{+} \cup\left(\bigcup_{k=0}^{n} \Pi_{k}^{+}\right) \cup\left(\bigcup_{k=0}^{n} H_{k}^{+}\right)=R_{n+1}^{+} \cup \Pi_{n+1}^{+} \cup H_{n+1}^{+}
$$

No entanto

$$
\bigcup_{k=0}^{n} \Pi_{k}^{+} \cup \bigcup_{k=0}^{n} H_{k}^{+} \subset \bigcup_{k=0}^{+\infty} \Pi_{k}^{+} \cup \bigcup_{k=0}^{+\infty} H_{k}^{+}
$$

Se um par $(v, \tau)$ é tal que

$$
(v, \tau) \notin \bigcup_{k=0}^{+\infty} \Pi_{k}^{+} \cup \bigcup_{k=0}^{+\infty} H_{k}^{+}
$$

então, o movimento correspondente $z(t, v, \tau)$ é obrigatoriamente oscilatório, isto é

$$
(v, \tau) \in R^{+}
$$

Daí com isso podemos fazer $n=+\infty$ na fórmula

$$
\Phi \backslash\{O\}=R_{n+1}^{+} \cup \Pi_{n+1}^{+} \cup H_{n+1}^{+}=\bigcap_{k=0}^{+\infty} R_{n} \cup\left(\bigcup_{k=0}^{n} \Pi_{k}^{+}\right) \cup\left(\bigcup_{k=0}^{n} H_{k}^{+}\right)=R^{+} \cup \Pi^{+} \cup H^{+}
$$

Analogamente temos também que

$$
\Phi \backslash\{O\}=R^{-} \cup \Pi^{-} \cup H^{-}
$$

Os conjuntos $H_{0}^{+}, H_{0}^{-}, R_{0}^{+} R_{0}^{-}$são abertos e $S$ é um homeomorfismo de $R_{0}^{+} \cup\{O\}$. Com isso, diretamente das sua respectivas definições, os conjunts $R_{n}^{+}, R_{n}^{-}, H_{n}^{+}, H_{n}^{-}$são todos conjuntos abertos e conseqüentemente os conjuntos $R^{+}, R^{-}, H^{+}, H^{-}$são do tipo $G_{\delta}$.

O conjunto $\Pi_{0}^{+}$é fechado, e dessa forma $\Pi_{0}^{+} \cap R_{0}^{+}$é relativamente fechado em $R_{0}^{-} \cap\{O\}$. Também $\Pi_{1}^{+}=S^{-1}\left(\Pi_{0}^{+} \cap R_{0}^{+}\right)$é relativamente fechado em $R_{0}^{+} \cap\{O\}$.

Daí temos que

$$
\Pi_{0}^{+} \cup \Pi_{1}^{+}=\Pi_{0}^{+} \cup\left(S^{-1}\left(\Pi_{0}^{+}\right) \cap\left(R_{0}^{+} \cup\{O\}\right)\right)=\left(\Pi_{0}^{+} \cup S^{-1}\left(\Pi_{0}^{+}\right)\right) \cap\left(\Pi_{0}^{+} \cup\left(R_{0}^{+} \cup\{O\}\right)\right)
$$

Como a fronteira $\partial\left(R_{0}^{+} \cup\{O\}\right)=\Pi_{0}^{+}$isto implica que $\left(R_{0}^{+} \cup\{O\}\right)$ é um conjunto fechado, $\operatorname{logo} \Pi_{0}^{+} \cup \Pi_{1}^{+}$é fechado. Por indução 


$$
\Pi_{0}^{+} \cup \cdots \cup \Pi_{n}^{+}
$$

é um conjunto fechado e então $\Pi^{+}$é do tipo $F_{\sigma}$.

Analogamente

$$
\Pi_{0}^{-} \cup \cdots \cup \Pi_{n}^{-}
$$

é um conjunto fechado e então $\Pi^{-}$é do tipo $F_{\sigma}$.

Da proposição acima, temos que todos os conjuntos em (37) e todos os seus análogos, com o sinal menos, são mensuráveis segundo Lebesgue. A medida $\mu=v d v d t$ é absolutamente contínua com respeito a medida de Lebesgue, $m$. Logo, os conjuntos da proposição acima são mensuráveis com respeito a $\mu$.

Proposição 2.7. Para todo $n \geq 1$ e $m \geq 0$ temos

$$
\begin{aligned}
& S\left(R^{+} \cap R^{-}\right)=R^{+} \cap R^{-} ; \quad S\left(R^{+} \cap \Pi_{m}^{-}\right)=R^{+} \cap \Pi_{m+1}^{-} ; \quad S\left(R^{+} \cap H_{m}^{-}\right)=R^{+} \cap H_{m+1}^{-} \\
& S\left(H_{n}^{+} \cap R^{-}\right)=H_{n-1}^{+} \cap R^{-} ; \quad S\left(H_{n}^{+} \cap \Pi_{m}^{-}\right)=H_{n-1}^{+} \cap \Pi_{m+1}^{-} ; \quad S\left(H_{n}^{+} \cap H_{m}^{-}\right)=H_{n-1}^{+} \cap H_{m+1}^{-} \\
& S\left(\Pi_{n}^{+} \cap R^{-}\right)=\Pi_{n-1}^{+} \cap R^{-} ; \quad S\left(\Pi_{n}^{+} \cap \Pi_{m}^{-}\right)=\Pi_{n-1}^{+} \cap \Pi_{m+1}^{-} ; \quad S\left(\Pi_{n}^{+} \cap H_{m}^{-}\right)=\Pi_{n-1}^{+} \cap H_{m+1}^{-}
\end{aligned}
$$

A prova da proposição segue diretamente da definição dos conjuntos envolvidos.

Teorema 2.4. Quase toda solução que é oscilatória para $t \rightarrow+\infty$ é oscilatória para $t \rightarrow$ $-\infty$. Quase toda solução parabólica ou hiperbólica para $t \rightarrow+\infty$ ainda continua parabólica ou hiperbólica para $t \rightarrow-\infty$.

Demonstração. A prova é trivial para o caso $\Im=+\infty$, pois neste caso como já visto no Teorema 4.1, todas as soluções de (14) são oscilatórias para $t \rightarrow \pm \infty$

Para o caso $\Im$ finito, pelo corolário 4.1 e proposição 4.7 , os conjuntos $R_{0}^{+}$e $R_{0}^{-}$tem medida $\mu$ finita, onde

$$
\mu\left(R_{0}^{ \pm}\right)=\int_{R_{0}^{ \pm}} v d v d t
$$


já que $\mu$ é absolutamente contínua com $m$, a medida de Lebesgue.

Para provar a primeira afirmação do teorema, temos que mostrar que $\mu\left(R^{+} \backslash R^{-}\right)=.0$

Definamos conjuntos $B_{m}=R^{+} \cap\left[H_{m}^{-} \cup \Pi_{m}^{-}\right], m \geq 0$. Por (39) temos que $S\left(B_{m}\right)=B_{m+1}$. Observe que

$$
\begin{aligned}
& R^{+} \backslash R^{-}=\bigcup_{m \leq 0}^{\cdot} B_{m} \\
& \mu\left(B_{m}\right)=\mu\left(B_{m+1}\right)
\end{aligned}
$$

como $S$ preserva a medida $\mu$

$$
\mu\left(R^{+} \backslash R\right)=\mu\left(\bigcup_{m \leq 0} B_{m}\right)=\sum_{m=0}^{+\infty} \mu\left(B_{m}\right)
$$

Como $\mu\left(B_{n}\right)=\mu\left(B_{m+1}\right)$ segue que $\mu\left(B_{m}\right)=0, m \geq 0$. Daí $\mu\left(R^{+} \backslash R^{-}\right)=0$.

O conjunto dos pontos do plano, cujas soçluções são parabólicas ou hiperbólicas para o futuro, mas não o são para o passado é dado por

$$
\bigcup_{m \geq 0}^{\cdot} R^{-} \cap\left[H_{m}^{+} \cup \Pi_{m}^{+}\right]=R^{-} \backslash R^{+} .
$$

De maneira análoga, provamos que $\mu\left(R^{-} \backslash R^{+}\right)=0$. o que completa a nossa demonstração.

Uma pergunta interessante diz respeito é quanto à medida dos conjuntos $R^{+}$e $R^{-}$. Sabemos que $S$ é um difeomorfismo que preserva, nas coordenadas polares o elemento de área $v d v d \tau$. Isso significa que $S$ preserva a medida de Lebesgue em $\mathbb{R}^{2}$. Assim, como $R^{-}=S\left(R^{+}\right)$ambos tem a mesma medida.

Sabemos que, independentemente da equação (14) ser autônomo, temos que os conjuntos $R_{0}^{ \pm}$são abertos e não vazios, como demonstrado no decorrer deste capítulo. No entanto, quando estamos tratando de equações autônomas, sabemos que a energia integral

$$
h(t)=\frac{\dot{z}(t)^{2}}{2}+\int_{0}^{z(t)} Q(y) d y
$$

é constante. Segue que neste caso

$$
h \equiv \frac{v}{2}^{2}
$$


Seja $(v, \tau) \in R_{0}^{+}$, isto significa que a solução que $z(t, v, \tau)$, esta deve ter um zero $\tau^{\prime}>\tau$. Estamos supondo $\tau^{\prime}$ o primeiro zero de $z(t, v, \tau)$, maior que $\tau$. Como $h$ é constante, $z(t, v, \tau)$ é diferenciável e $v>0$, temos que

$$
\dot{z}\left(\tau^{\prime}, v, \tau\right)=-v
$$

isso significa que passado tempo $\tau^{\prime}-\tau$ a solução $z(t, v, \tau)$ se anula.

Do teorema de existência e unicidade de soluções de equações diferenciais ordinárias de das simetrias em (17), temos que

$$
z(t, v, \tau)=z\left(t,-v, \tau^{\prime}\right)=-z\left(t, v, \tau^{\prime}\right)
$$

Como estamos supondo tratar-se de um sistema autônomo, temos que

$$
z(t-\tau, v, 0)=z(t, v, \tau)=z\left(t,-v, \tau^{\prime}\right)=-z\left(t-\tau^{\prime}, v, 0\right)=-z\left(t-\tau+\left(\tau^{\prime}-\tau\right), v, 0\right) .
$$

Somando $\tau^{\prime}-\tau$ na primeira e na última parcela acima obtemos:

$$
-z\left(t-\tau^{\prime}, v, 0\right)=-z\left(t-\tau+2\left(\tau^{\prime}-\tau\right), v, \tau\right)
$$

$\operatorname{mas} z\left(t-\tau^{\prime}, v, 0\right)=z(t-\tau, v, 0)$

Com isso

$$
z(t-\tau, v, 0)=z\left(t-\tau+2\left(\tau^{\prime}-\tau\right), v, 0\right) .
$$

Assim, toda solução que se anula em mais de um ponto é periódica de período $\tau^{\prime}-\tau$, como feito acima. Desta maneira temos a igualdade:

$$
R^{+}=R_{0}^{+}=R_{0}^{-}=R^{-}
$$

E assim, o conjunto das trajetórias oscilatórias tem medida positiva, pois $R^{+}=R^{-}$são abertos e não vazios.

No problema de Sitnikov, quando $\varepsilon=0$, temos a seguinte configuração: 


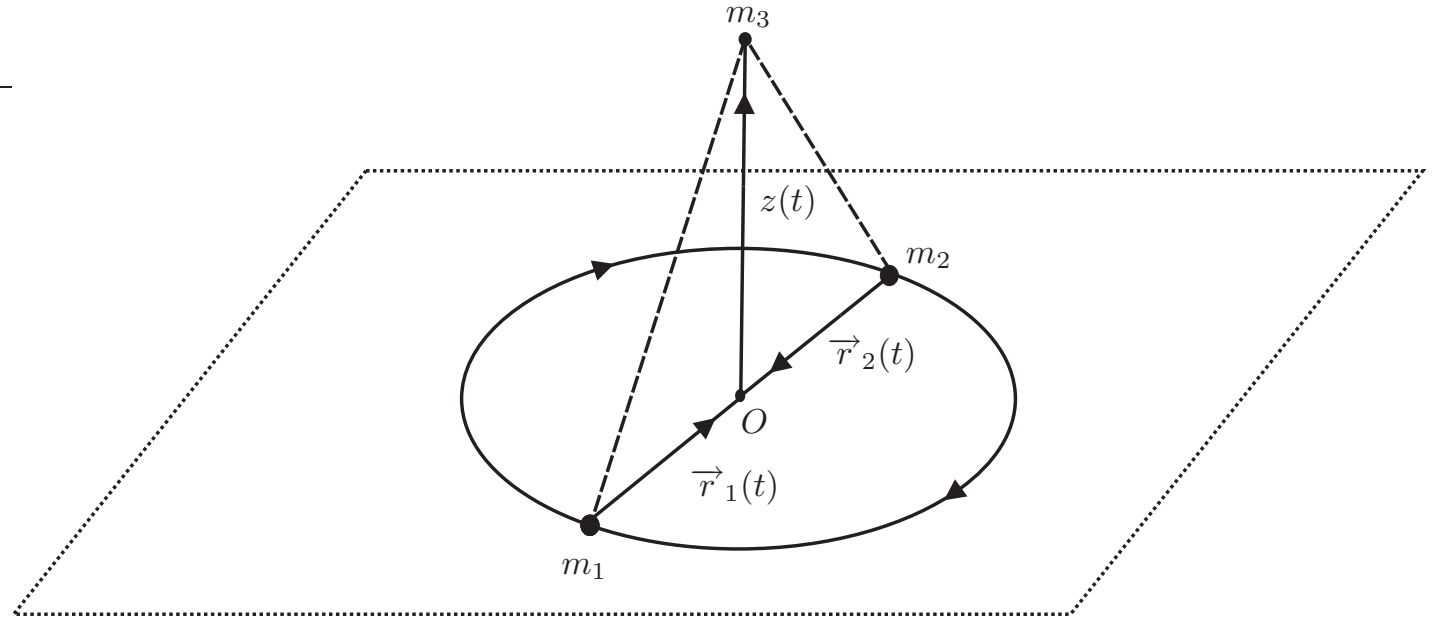

Figura 5: Configuração do Problema, quando $\varepsilon=0$

Observemos também que no caso autônomo quando $\varepsilon=0$, por termos

$$
h(t)=\frac{\dot{z}(t)^{2}}{2}+\int_{0}^{z(t)} Q(y) d y
$$

uma função constante, segue que qualquer movimento $z(t, v, \tau)$ que satisfaça:

$$
\frac{v}{2}^{2}=\Im
$$

corresponde a uma trajetória parabólica, pelo teorema 2.1. No caso do problema de Sitnikov, temos que

$$
\Im=\int_{0}^{+\infty} \frac{z}{\left(z^{+}+1\right)^{3 / 2}}=-\left.\frac{1}{\sqrt{z^{2}+1}}\right|_{0} ^{+\infty}=1
$$

Assim, qualquer condição inicial que tenhamos

$$
\frac{v}{2}^{2}=1
$$

temos uma solução parabólica. Assim, para o caso autônomo do problema de Sitnikov tanto $\Pi_{0}^{+}$quanto $\Pi_{0}^{-}$são circunferências de centro na origem e raio $\sqrt{2}$.

Sendo assim, os conjuntos $R^{+}$e $R^{-}$no problema de Sitnikov tem a mesma medida do círculo de raio $\sqrt{2}$, como já sabemos da igualdade

$$
R^{+}=R^{-}=R_{0}^{+}=R_{0}^{-}
$$


Como variaria a medida de tais conjuntos, $R^{+}$e $R^{-}$por uma $\varepsilon$-perturabação $C^{1}$ do problema de Sitnikov?

Com respeito a essa questão, temos a seguinte conjectura, devida a Kolmogorov.

Conjectura 2.1 (Kolmogorov). A medida dos conjuntos, $R^{+}$e $R^{-}$para perturbações $C^{1}$ de equações do tipo (14) é nula. 


\section{$3 \quad$ Nascimento da Ferradura de Smale}

\subsection{Uma Visão Geométrica do Problema Restrito dos Três Corpos}

\subsubsection{Método de Melnikov para Pontos Homoclínicos de Sistemas Hamiltonianos Perturbados}

Exibiremos nesta secção um método(método de Melnikov), com o qual podemos encontrar intersecções homoclínicas transversais para perturabações a um parâmetro de Sistemas Hamiltonianos planares. Este método também é conhecido na literatura como método de PoincaréMelnikov-Arnold. O simples e original contexto do método de Melnikov envolve perturabações periódicas de Sistemas Hamiltonianos.

Conseideremos, neste caso um Hamiltoniano $H: \mathbb{R}^{2} \rightarrow \mathbb{R}$ de classe $C^{2}$ e o sistema de equações Hamiltonianas.

$$
\left\{\begin{array}{l}
\dot{p}=-\frac{\partial H}{\partial q}=-H_{q} \\
\dot{q}=\frac{\partial H}{\partial p}=H_{p}
\end{array}\right.
$$

Chamamos $x=(p, q)$, e $X_{H}=\left(-H_{q}, H_{p}\right)$ e a equação acima pode ser reescrita da seguinta forma:

$$
\dot{x}=X_{H}(x)
$$

Observe que para todo $x \in \mathbb{R}^{2}$ o campo $X_{H}$ é ortogonal ao campo gradiente $\nabla H=\left(H_{p}, H_{q}\right)$. Por outro lado, sabemos que os vetores tangentes a cada curva de nível $H(p, q)=$ cte são ortogonais ao gradiente $\nabla H$, assim, como o Hamiltoniano é bi-dimensional, as curvas soluções de (13) estão sobre as curvas de nível $H(p, q)=$ cte.

Consideremos agora a seguinte perturbação do campo Hamiltoniano $X_{H}$

$$
X_{\varepsilon}=X_{H}+\varepsilon Y(p, q, t)
$$

tal que $Y(p, q, t)$ é periódico em $t$, de período $T$. Estamos supondo também que $Y$ é $C^{2}$ em suas coordenadas. A partir daí, vamos considerar o seguinte sistema autônomo de equações diferenciais. 


$$
\left\{\begin{array}{c}
\dot{x}=X_{\varepsilon} \\
\dot{\tau}=1
\end{array}\right.
$$

onde $\tau$ é uma variável angular tomada $\tau(\bmod T)$. Colocarem $\widehat{X}_{\varepsilon}=\left(X_{\varepsilon}, 1\right)$

Assumiremos que $X_{H}$ exiba por $(0,0)$ um ponto de sela hiperbólica e homoclínico.

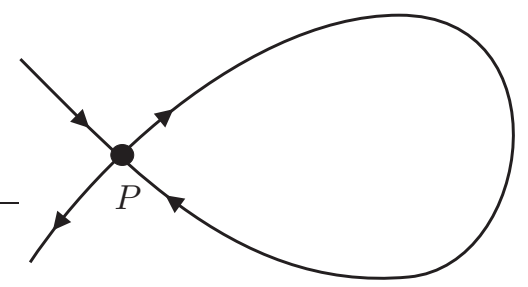

Figura 6: laço de sela

Neste caso estamos assumindo a existência de um laço de Sela, de modo que para $\varepsilon=0$, a equação (42) possua uma órbita fechada passando pela origem.

Chamaremos tal trajetória de $\gamma_{0}$. Para $\varepsilon$ pequeno, a equação (42) exibe uma órbita fechada que passando por uma singularidade do tipo sela hiperbólica do campo $X_{\varepsilon}$ que está prómimo a $P_{0}=(0,0)$. A existência desta nova singularidade segue do teorema da função implícita, desde que

$$
\frac{\partial X_{\varepsilon}}{\partial \varepsilon}(0,0, \varepsilon=0) \neq 0
$$

e a hiperbolicidade, segue da abertura dos sistemas hiperbólicos.

A essa órbita fechada de $\widehat{X}_{\varepsilon}$ chamaremos de $\gamma_{\varepsilon}$.

Seja $\Gamma=\{(p, q, \tau) \mid(p, q)$ está sobre o laço homoclinico de $(0,0)$ do campo hamiltoniano $X\}$

Vamos supor que $\nabla H$ não se anule sobre os pontos de $\Gamma$ projetados no plano $(p, q)$.

Para $\varepsilon$ suficientemente pequeno, as variedades estáveis e instáveis de $\gamma_{\varepsilon}$ variam diferenciavelmente com $\varepsilon$ em subconjuntos compactos.

Para cada $z_{0} \in \Gamma$ seja $z^{s}\left(z_{0}, \varepsilon\right)$ o ponto onde $W^{s}\left(\gamma_{\varepsilon}, \widehat{X}_{\varepsilon}\right)$ intersecta a reta normal a $\Gamma$ passando por $z_{0}$. Coloquemos $z^{s}\left(z_{0}, 0\right)=z_{0}$. Analogamente definimos o ponto $z^{u}\left(z_{0}, \varepsilon\right)$. Por dependência diferenciável em $\varepsilon, z^{s}$ e $z^{u}$ definidos sobre $\Gamma$ são funções diferenciáveis em $\varepsilon$. Queremos medir a separação das variedades estáveis e instáveis nas direções ortogonais a $\Gamma$, em outras palavras queremos medir a separação dos pontos $z^{u}\left(z_{0}, \varepsilon\right)$ e $z^{s}\left(z_{0}, \varepsilon\right)$. 
O espaço tangente a $\Gamma$ num ponto $z_{0}$ é gerado pelos vetores $\left\{\left(-H_{q}, H_{p}, 0\right),(0,0,1)\right\}$, sendo assim o vetor normal ao espaço tangente é dado por $\left(H_{p}, H_{q}, 0\right)=(\nabla H, 0)$. Definiremos então a função de displaçamento entre $z^{u}\left(z_{0}, \varepsilon\right)$ e $z^{s}\left(z_{0}, \varepsilon\right)$. Para simplificar notação, a nomenclatura $z^{u}\left(z_{0}, \varepsilon\right)$ e $z^{s}\left(z_{0}, \varepsilon\right)$ também se refere à projeção dos mesmos no plano $(p, q)$. Sendo assim, definimos:

$$
\widehat{G}\left(z_{0}, \varepsilon\right)=H\left(z^{u}\left(z_{0}, \varepsilon\right)\right)-H\left(z^{s}\left(z_{0}, \varepsilon\right)\right)
$$

$\widehat{G}$ é chamada de função de displaçamento.

Observe que $\widehat{G}$ é diferenciável com respeito a $\varepsilon$.

Se $\varepsilon$ for suficientemente pequeno, ambos $z^{u}$ e $z^{s}$ estão suficientemente próximos de $z_{0}$ e no plano $(p, q)$ o vetor $\left(z^{u}\left(z_{0}, \varepsilon\right)-z^{s}\left(z_{0}, \varepsilon\right)\right)$ é um múltiplo do gradiente $\nabla H$, pois estão sobre a reta normal a $\Gamma$ por $z_{0}$, que tem como vetor diretor $(\nabla H, 0)$.

Quando $\varepsilon=0$ temos:

$$
\widehat{G}\left(z_{0}, 0\right) \equiv 0
$$

sendo assim podemos escrever

$$
\widehat{G}\left(z_{0}, \varepsilon\right)=H\left(z^{u}\left(z_{0}, \varepsilon\right)\right)-H\left(z^{s}\left(z_{0}, \varepsilon\right)\right)=\varepsilon G\left(z_{0}, \varepsilon\right)
$$

Um zero de $G\left(z_{0}, \varepsilon\right)$ corresponde a um ponto homoclínico, de fato.

Se temos um ponto homoclínico para $X_{\varepsilon}$, então temos que $z^{u}\left(z_{0}, \varepsilon\right)=z^{s}\left(z_{0}, \varepsilon\right)$ e daí $G\left(z_{0}, \varepsilon\right)=0$. Reciprocamente, se $G\left(z_{0}, \varepsilon\right)=0$ temos, pelo teorema do valor médio que,

$$
0=H\left(z^{u}\left(z_{0}, \varepsilon\right)\right)-H\left(z^{s}\left(z_{0}, \varepsilon\right)\right)=\left\langle\nabla H(\bar{z}),\left(z^{u}-z^{s}\right)\right\rangle=\left\langle\lambda \nabla H\left(z_{0}\right), \nabla H(\bar{z})\right\rangle
$$

para algum $\lambda \in \mathbb{R}$, e $\bar{z}$ é um ponto sobre o caminho ligando $z^{u}$ a $z^{s}$.

Como $\nabla H$ não se anula sobre o laço homoclínico de $X$ e $\varepsilon$ é suficientemente pequeno, temos que

$$
\left\langle\nabla H\left(z_{0}\right), \nabla H(\bar{z})\right\rangle \approx\left\|\nabla H\left(z_{0}\right)\right\|^{2} \neq 0
$$

daí $\lambda=0$ com isso 


$$
\left(z^{u}-z^{s}\right)=\lambda \nabla H\left(z_{0}\right)=0 \Rightarrow z^{u}=z^{s} .
$$

isso mostra que temos uma intersecção homoclínia para os zeros de $\widehat{G}$.

Queremos medir a taxa de variação de $\widehat{G}$ com respeito a $\varepsilon$ e definimos:

$$
M\left(z_{0}\right)=\left.\frac{\partial}{\partial \varepsilon} H\left(z^{u}\left(z_{0}, \varepsilon\right)\right)\right|_{\varepsilon=0}-\left.\frac{\partial}{\partial \varepsilon} H\left(z^{s}\left(z_{0}, \varepsilon\right)\right)\right|_{\varepsilon=0}
$$

observe que

$$
M\left(z_{0}\right)=G\left(z_{0}, 0\right)
$$

$M\left(z_{0}\right)$ definida em $\Gamma$ e assumindo valores reais, é chamada função de Melnikov. Com isso, $G\left(z_{0}, \varepsilon\right)$ é igual a $M\left(z_{0}\right)$ mais termos em $\varepsilon$. Um zero de $M$ corresponde a um lugar infinirezimal onde as variedades estáveis e instáveis continuam se tocando, para o sistema perturbado.

O teorema que segue é uma conseqüência do teorema da função implícita, nos dá um critério, tal que as variedades estáveis e instáveis do sistema perturbado, intersectam transversalmente. O uso dessa função para provar a existência de pontos homoclínicos transversais e uma ferradura de Smale é referido como uma amplicação do Método de Melnikov.

Teorema 3.1. Suponha que $z_{0}$ é um ponto sobre $\Gamma$ com $M\left(z_{0}\right)=0$ e alguma derivada direcional $\frac{\partial M}{\partial v}\left(z_{0}\right) \neq 0$ para algum vetor $v$ tangente a $\Gamma$. Então para todo $\varepsilon \neq 0$ suficientemente pequeno, as variedades estáveis e instáveis de $\gamma_{\epsilon}$ tem uma instersecção transversal numa vizinhança de $z_{0}$, e tais pontos variam continuamente com $\varepsilon$.

Como conseqüência desse teorema, o campo perturbado $\widehat{X}_{\varepsilon}$ tem uma ferradura de Smale numa vizinhança do ponto $z_{0}$.

Teorema 3.2. A função de Melnikov é dada pela seguinte integral imprópria

$$
M\left(z_{0}\right)=\int_{-\infty}^{+\infty} D H_{\phi_{0}\left(t, z_{0}\right)} Y\left(\phi_{0}\left(t, z_{0}\right)\right) d t
$$

onde $\phi_{0}\left(t, Z_{0}\right)$ é o fluxo com condições iniciais em $z_{0}$ para $t=0$ da equação (41). 
Demonstração. Precisamos calcular

$$
\frac{\partial}{\partial \epsilon} H \circ \phi\left(t, z^{\sigma}\left(z_{0}, \varepsilon\right)\right)
$$

para $\sigma \in\{u, s\}$.

Pelo teorema de Schwarz temos que

$$
\begin{gathered}
\left.\frac{d}{d t} \frac{\partial}{\partial \epsilon} H \circ \phi\left(t, z^{\sigma}\left(z_{0}, \varepsilon\right)\right)\right|_{\varepsilon=0}=\left.\frac{\partial}{\partial \varepsilon} \frac{d}{d t} H \circ \phi\left(t, z^{\sigma}\left(z_{0}, \varepsilon\right)\right)\right|_{\varepsilon=0}= \\
\frac{\partial}{\partial \varepsilon}\left(\left.D H\left(\phi\left(t, z^{\sigma}\left(z_{0}, \varepsilon\right), \varepsilon\right)\right)\left(X_{H}+\varepsilon Y\right)\right|_{\varepsilon=0}=D H . Y+\frac{\partial}{\partial \varepsilon}\left(D H . X_{H} \equiv 0\right)=D H . Y_{\phi_{0}\left(t, z_{0}\right)}\right.
\end{gathered}
$$

integrando em $t$, entre $T_{1}$ e $T_{2}$ nós encontramos:

$$
\left.\frac{\partial}{\partial \varepsilon} H \circ \phi\left(T_{2}, z^{\sigma}\left(z_{0}, \varepsilon\right), \varepsilon\right)\right|_{\varepsilon=0}-\left.\frac{\partial}{\partial \varepsilon} H \circ \phi\left(T_{1}, z^{\sigma}\left(z_{0}, \varepsilon\right), \varepsilon\right)\right|_{\varepsilon=0}=\int_{T_{1}}^{T_{2}} D H . Y_{\phi_{0}\left(t, z_{0}\right)} d t
$$

Para $\sigma=s$ usamos $T_{1}=0$ e $T_{2}=T_{2}^{\prime} \rightarrow+\infty$ e para $\sigma=u$ usamos $T_{2}=0$ e $T_{1}=T_{1}^{\prime} \rightarrow-\infty$ lembrando que

$$
M\left(z_{0}\right)=\left.\frac{\partial}{\partial \varepsilon} H\left(z^{u}\left(z_{0}, \varepsilon\right)\right)\right|_{\varepsilon=0}-\left.\frac{\partial}{\partial \varepsilon} H\left(z^{s}\left(z_{0}, \varepsilon\right)\right)\right|_{\varepsilon=0}
$$

Chamando

$$
\begin{aligned}
& I_{1}=\int_{T_{1}=0}^{T_{2}^{\prime}} D H . Y_{\phi_{0}\left(t, z_{0}\right)} d t=\left.\frac{\partial}{\partial \varepsilon} H \circ \phi\left(T_{2}^{\prime}, z^{s}\left(z_{0}, \varepsilon\right), \varepsilon\right)\right|_{\varepsilon=0}-\frac{\partial}{\partial \varepsilon} H\left(\left.z^{s}\left(z_{0}, \varepsilon, \varepsilon\right)\right|_{\varepsilon=0}\right. \\
& I_{2}=\int_{T_{1}^{\prime}}^{T_{2}=0} D H . Y_{\phi_{0}\left(t, z_{0}\right)} d t=\left.\frac{\partial}{\partial \varepsilon} H\left(z^{u}\left(z_{0}, \varepsilon\right)\right)\right|_{\varepsilon=0}-\left.\frac{\partial}{\partial \varepsilon} H \circ \phi\left(T_{1}^{\prime}, z^{u}\left(z_{0}, \varepsilon\right), \varepsilon\right)\right|_{\varepsilon=0}
\end{aligned}
$$

observe que $\phi\left(T_{2}^{\prime}, z_{0}, 0\right) \rightarrow P=(p, q)=(0,0)$ quando $T_{2}^{\prime} \rightarrow+\infty, D H_{P}=0$, de forma que que $D H_{\phi\left(T_{2}^{\prime}, z_{0}, 0\right)} \rightarrow 0$ quando $T_{2}^{\prime} \rightarrow+\infty$.

Por outro lado $\phi\left(T_{2}^{\prime}, z_{0}, \varepsilon\right) \rightarrow \gamma_{\varepsilon}$, órbita fechada, quando $T_{2}^{\prime} \rightarrow+\infty$. Daí pela continuidade diferencial $\frac{\partial}{\partial \varepsilon} \phi\left(T_{2}^{\prime}, z^{s}\left(z_{0}, \varepsilon, \varepsilon\right)\right)$ é limitada quando $T_{2}^{\prime} \rightarrow+\infty$.

Assim, usando a regra da cadeia, vemos que quando $T_{2}^{\prime} \rightarrow+\infty$ temos o seguinte

$$
I_{1} \rightarrow-\frac{\partial}{\partial \varepsilon} H\left(\left.z^{s}\left(z_{0}, \varepsilon, \varepsilon\right)\right|_{\varepsilon=0}\right.
$$


e um raciocínio análogo na análise de $I_{2}$, vemos que quando integrais $T_{1}^{\prime} \rightarrow-\infty$ segue que

$$
I_{2} \rightarrow \frac{\partial}{\partial \varepsilon} H\left(\left.z^{u}\left(z_{0}, \varepsilon, \varepsilon\right)\right|_{\varepsilon=0}\right.
$$

somando $I_{1}$ e $I_{2}$ em seus limites, obtemos (43) como desejado.

\subsection{Existência de Pontos Homoclínicos Transversais no Problema de Sitnikov}

Nesta secção, utilizaremos o Método de Melnikov descrito anteriormente para encontrar pontos homoclínicos transversais no problema de Sitnikov, obtendo assim, na vizinhança de tal ponto um dinâmica de Ferradura de Smale.

Segundo a nossa formulação do problema dos três corpos com a configuração como abaixo:

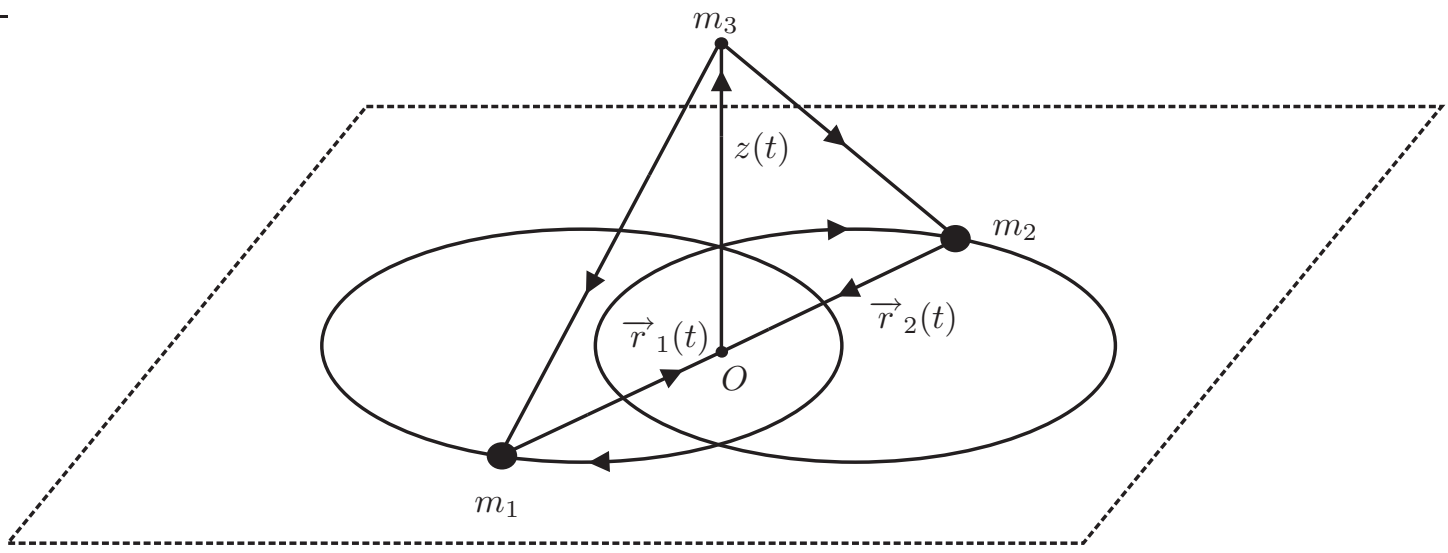

Figura 7: Configuração do Problema

Seja $z(t)$ a posição da partícula $m_{3}$ com relação ao tempo, sobre a reta $L$ normal plano de movimento dos corpos primários, $m_{1}$ e $m_{2}$ a saber. Temos que a equação diferencial que rege o movimento de $m_{3}$ é dado por

$$
\ddot{z}(t)=-\frac{z(t)}{\left(z^{2}(t)+r(t)^{2}\right)}
$$

tal que $r$ é par e analítica na variável $t$.

$$
r(t)=1+\varepsilon \cos t+O\left(\varepsilon^{2}\right)
$$


onde $\varepsilon$ é a excentricidade das elipses onde movem-se os corpos primários. Note que se $\varepsilon=0$ estamos no caso de um problema circular $\left(r=r_{0}=1 / 2\right)$, autônomo e conservativo.

Chame

$$
Q(z, t)=\frac{z(t)}{\left(z^{2}(t)+r(t)^{2}\right)}
$$

e a equação (44) pode ser transformada no seguinte sistema

$$
\left\{\begin{array}{lc}
\dot{z}= & y \\
\dot{y}= & -Q(z, t)
\end{array}\right.
$$

a função $Q(z, t)$ da equação diferencial (44) tem as propriedades descritas no capítulo 2 , "Estudo Qualitativo de $\ddot{z}=-Q(z, t)$ " além de ser ímpar em $z$.

Logo as soluções $(z(t), y(t))$ de $(45)$ são invariantes pelas simetrias

$$
(z, y, t) \rightarrow(-z, y,-t), \quad(z, y, t) \rightarrow(-z,-y, t), \quad(z, y, t) \rightarrow(z,-y,-t)
$$

Com isso podemos restringir as condições iniciais do sistema (45) ao primeiro quadrante do plano $z y$.

No caso $\varepsilon=0$ temos que o sistema (45) é um sistema hamiltoniano com hamiltoniano $H: \mathbb{R}^{2} \rightarrow \mathbb{R}$ dado por:

$$
H(z, y)=\frac{y}{2}^{2}-\frac{1}{\sqrt{z^{2}+1}}
$$

Estamos no contexto que queríamos, conforme a secção anterior. Vamos estudar as perturbações deste Hamiltoniano, variando o parâmetro $\varepsilon$. No caso autônomo, conhecemos completamente a dinâmica, e veremos que para $\varepsilon$ tão pequeno quanto se queira a equação (45) tem dinâmica muito diferente do sistema hamiltoniano não perturbado.

Já sabemos que para o problema de Sitnikov existem, além da órbita estacionária, somente três outros tipos a saber parabólicas, hiperbólicas e oscilatórias.

Observe que

$$
H \equiv 0 \Rightarrow z^{2}=+\infty \quad \text { e } \quad y=0
$$


Logo o nível de energia zero do hamiltoniano $H$ equivale às condições inicias das trajetórias parabólicas, tais condições estão sobre a curva a saber:

$$
\frac{d z}{d t}=y=\frac{\sqrt{2}}{\left(z^{2}+1\right)^{1 / 4}}
$$

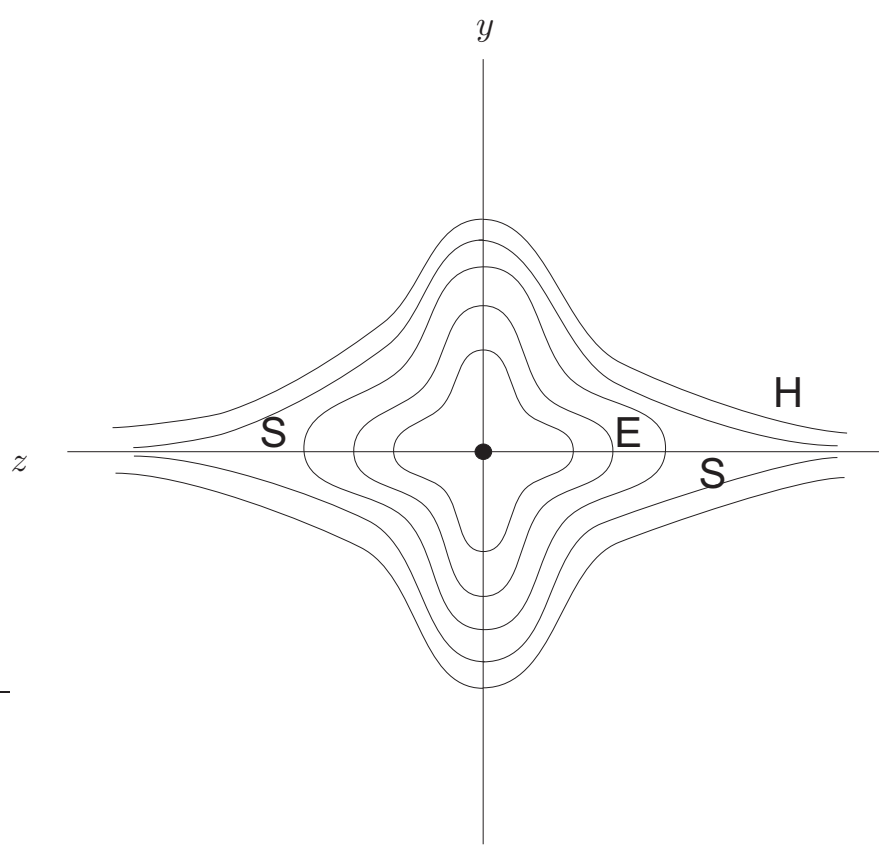

Figura 8: (S) indica a separatriz entre as órbitas elípticas (E), e hiperbólicas (H)

As órbitas elípticas são as que tem pelo menos um zero $\tau^{\prime}>\tau$.

\subsubsection{As Transformações de McGehee}

Introduziremos agora as seguintes coordenadas devidas a McGehee

$$
z=\frac{2}{q^{2}}, \quad \dot{z}=-p, \quad \frac{d t}{d s}=\frac{4}{q^{3}}
$$

Com $0<q<+\infty$, observe que quando $q \rightarrow 0$ correstponde a $z \rightarrow+\infty$.

Derivando a primeira das igualdades em (48) com respeito a $s$, obtemos:

$$
-\frac{4}{q^{3}} \frac{d q}{d s}=\frac{d z}{d s}=\frac{d z}{d t} \frac{d t}{d s}=-p \frac{4}{q^{3}} \Rightarrow \frac{d q}{d s}=p
$$

Derivando a segunda das igualdades em (55) com respeito a $s$ : 


$$
\frac{d p}{d s}=-\ddot{z} \frac{d t}{d s}=\frac{z}{\left[z^{2}+r^{2}\right]^{3 / 2}} \frac{4}{q^{3}}=\frac{2}{q^{2}\left[\frac{4}{q^{4}}+r^{2}\right]^{3 / 2}} \frac{4}{q^{3}}=\frac{q}{\left[1+\frac{q^{4} r^{2}}{4}\right]^{3 / 2}}
$$

Após estes cálculos obtemos o seguinte sistema.

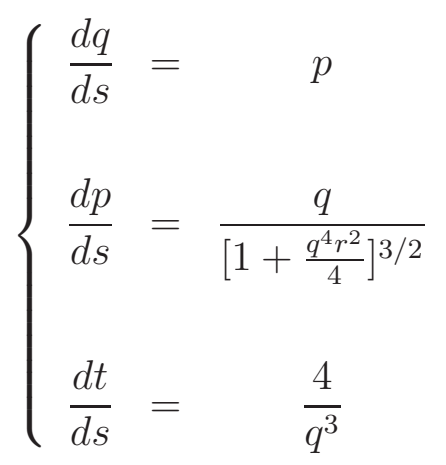

No caso autônomo podemos esquecer por a $t$ dependência do sistema acima. As duas primeiras igualdades de (49) nos dão um campo de vetores $X(p, q)$ o qual queremos estudar o retrato de fase das soluções numa vizinhança de sua singularidade $O=(0,0)$. Calculando a matriz Jacobiana do campo $X$ na origem $O$, encontramos

$$
D X(0,0)=\left(\begin{array}{ll}
0 & 1 \\
1 & 0
\end{array}\right)
$$

Que tem autovalores \pm 1 , logo, no sentido de campos de vetores, pelo teorema de GrobmanHartman, $(0,0)$ é uma singularidade do tipo sela hiperbólica, e suas variedades estáveis e instáveis numa vizinhança da origem são dadas por $q= \pm p+\ldots$ duas séries em $p$.

Observe que ao passarmos para as coordenadas de McGehee, a sela hiperpólica $(p, q)=$ $(0,0)$ do sistema não perturbado, cooresponde a uma sigularidade no infinito para o sistema hamiltoniano não perturbado original, dada em (45), com hamiltoniano conforme (47). Com isso a órbita homoclínica que passa por $z=0$ não é compacta.

Para tratar desse problema introduziremos agora uma mudanã de coordenadas não trivial

$$
z=\tan u, \quad v=\dot{z}=y, \quad u \in\left[-\frac{\pi}{2}, \frac{\pi}{2}\right], \quad v \in \mathbb{R}
$$

Com isso temos que:

$$
\dot{u}=v \cos ^{2} u
$$

de fato, derivando obtemos: 


$$
\dot{z}=\dot{u} \frac{1}{\cos ^{2}} \Rightarrow \dot{u}=\dot{z} \cos ^{2} u=\dot{u}=v \cos ^{2} u
$$

quando substituímos na equação (45) obtemos

$$
\left\{\begin{array}{lcc}
\dot{u} & = & v \cos ^{2} u \\
\dot{v} & = & -\frac{\tan u}{\left(\tan ^{2} u+r^{2}\right)^{3 / 2}}
\end{array}\right.
$$

Substituindo as fórmulas em (50) no hamiltoniano $H$ obtemos

$$
H(u, v)=\frac{1}{2} v^{2}-\frac{1}{\sqrt{\tan ^{2} u+r^{2}}}=H_{0}(u, v)+\varepsilon H_{1}(u, v, t, \varepsilon)
$$

quando $\varepsilon=0$ este reduz a

$$
H(u, v)=H_{0}(u, v)=\frac{1}{2} v^{2}-\frac{1}{\sqrt{\tan ^{2} u+1}}=\frac{1}{2} v^{2}-\cos u
$$

observe que o sistema (51) mesmo com $\varepsilon=0$ não é hamiltoniano.

Observe que para $H_{0}$ é constante ao longo das soluções de (51) quando $\varepsilon=0$. De fato, sejam $(u(t), v(t))$ soluções de $(51)$ daí temos que:

$$
\frac{d}{d t} H_{0}(u, v)=v \dot{v}+\sin u \dot{u}=-v \sin u \cos ^{2} u+v \sin u \cos ^{2} u=0
$$

Por simples aplicação da regra da cadeia vemos que $\nabla H_{0}$ é ortogonal ao campo definido em (51), e tempos que a demonstração do método de Melnikov é aplicável também neste caso.

A equação (53) é retrato de um movimento do tipo pêndulo com duas ligações de selas degeneradas, os pontos $\left( \pm \frac{\pi}{2}, 0\right)$ que equivalem ao ponto no infinito $(0,0)$ das transformações de McGehee. Essas novas transformações deixam compactas as variedades estáveis e instáveis da sela hiperhólica no infinito de (45).

Nós agora colocaremos $\varepsilon \neq 0$ e estudaremos o sistema nas coordenadas $(u, v)$. A singularidade $(0,0)$ torna-se uma órbita periódica $\gamma$ desde que introduzimos $t$ como variável angular $t(\bmod 2 \pi)$. Segundo um teorema de McGehee, nós concluímos que a variedade estável e instável desta órbita periódica é uma variedade real e analítica, as quai denotaremos por $W^{s}(\gamma)$ e $W^{u}(\gamma)$ respectivamente. 


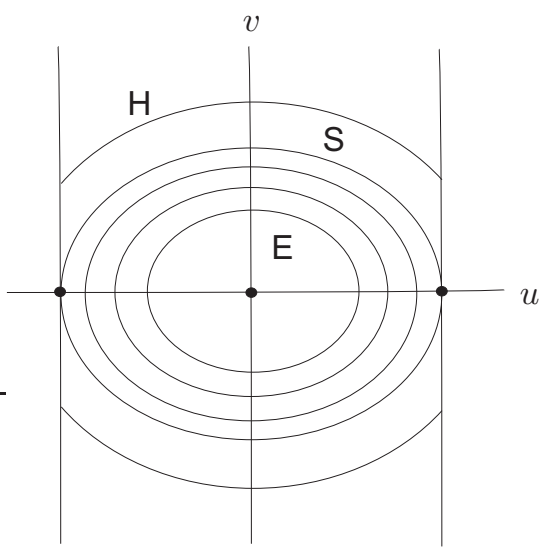

Figura 9: (S) indica a separatriz entre as órbitas elípticas (E), e hiperbólicas (H)

As simetrias dadas em (46) nos mostra que tais variedades se intersectam para $z=0$ quando $\varepsilon \neq 0$. Isto segue do fato de uma escolha conveniente de uma velocidade inicial que tal que $z(0)=0=z(+\infty)=0$. Então esta órbita mora em $W^{s}(\gamma)$. A primeira das simetrias dadas em (46) nos mostra que tal solução satisfaz que $z(t)=-z(-t)$ daí esta também mora sobre $W^{u}(\gamma)$

\subsubsection{Método de Melnikov aplicado ao Problema Restrito dos Três Corpos}

Teorema 3.3. Definamos a função de Melnikov

$$
M\left(t_{0}\right)=\int_{-\infty}^{+\infty}\left[\frac{d H_{1}}{d t}\left(u_{0}\left(t-t_{0}\right), v_{0}\left(t-t_{0}\right), t\right)\right]_{\varepsilon=0} d t
$$

Se $M\left(t_{0}\right)$ tem zeros simples então para $\varepsilon>0$ suficientemente pequeno, $W^{u}(\gamma)$ e $W^{s}(\gamma)$ intersectam-se transversalmente.

Demonstração. Com respeito a $H$ dada em (52) encontramos

$$
\begin{gathered}
\frac{d H}{d t}=v \dot{v}+\frac{r \dot{r}+\tan u\left(1+\tan ^{2} u\right)}{\left(\tan ^{2} u+r^{2}\right)^{3 / 2}}=v \dot{v}+\dot{u} \sin u+\varepsilon \frac{d H_{1}}{d t}(u, v, t, \varepsilon)= \\
v \dot{v}+\frac{r \dot{r}+\tan u\left(1+\tan ^{2} u\right)}{\left(\tan ^{2} u+r^{2}\right)^{3 / 2}}-\varepsilon \sin t-\varepsilon\left(\frac{\tan u\left(1+\tan ^{2} u\right) \dot{u}}{\left(1+\tan ^{2} u+1\right)^{3 / 2}} \cdot 3 r \cos u \frac{d r}{d \varepsilon}\right)= \\
=v \dot{v}+\dot{u} \sin u-\varepsilon \frac{\sin t}{\left(1+\tan ^{2} u\right)^{3 / 2}}
\end{gathered}
$$

Daí a equação de Melnikov ganha a forma 


$$
M\left(t_{0}\right)=-\int_{-\infty}^{+\infty} \frac{\sin \left(t-t_{0}\right)}{\left(1+\tan ^{2} u_{0}\right)^{3 / 2}} d t
$$

Seja $z_{0}(t)$ uma solução de (41) que se anula em $t=0$ e é parabólica. Pelas simetrias em (46), $z_{0}(t)=-z_{0}(t)$ o mesmo acontece com $u_{0}$ pelas transformações (50), logo o integrando em (54) é impar e daí $M(0)=0$. Temos ainda que

$$
M^{\prime}\left(t_{0}\right)=-\int_{-\infty}^{+\infty} \frac{\cos \left(t-t_{0}\right)}{\left(1+\tan ^{2} u_{0}\right)^{3 / 2}} d t=-\int_{-\infty}^{+\infty} \frac{\cos \left(t-t_{0}\right)}{\left(1+z_{0}(t)^{2}\right)^{3 / 2}} d t
$$

Como o integrando em (56) é par basta analisá-lo com limites de integração 0 e $+\infty$.

Lembremos que $z_{0}(t)$ é monótona e tende a infinito (menos infinito) quando $t$ tende a infinito (menos infinito), daí pelo critério da série alternada a integral indefinida dada em (56) converge a $\alpha>0$

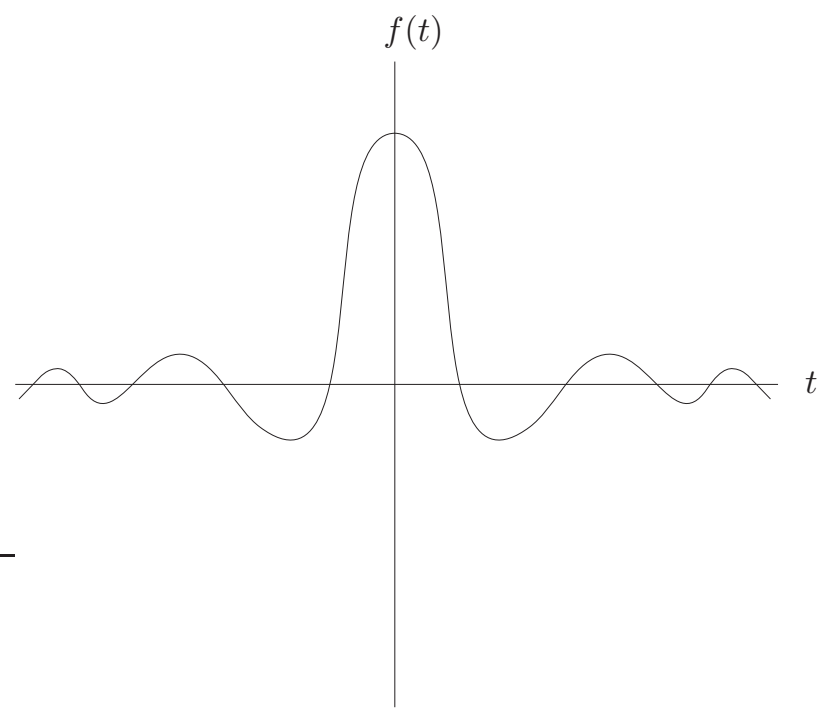

Figura 10: $f(t)$ indica o integrando em (56).

Com relação a interseções homiclínicas para o caso de trajetórias de campos vetorias temos Teorema 3.4 (Um Teorema de Smale-Birkhoff Modificado). As interseções homoclínicas transversais das variedades estáveis e instáveis, da aplicação de Poincaré, sobre outros pontos fixos da da equação (51), implica a existência de um conjunto hiperbólico invariante I, sobre o qual a dinâmica da aplicação de Poincaré é topologicamente equivalente a um shift no espaço das seqüências bi-infinitas, de finitos símbolos. 


\subsection{A Dinâmica $S$ Possui um Shift como subsistema}

Para esta seção usaremos os resultado do capítulo seguinte, os quais serão aqui assumidos. Para a nossa proposta, ainda precisaremos de alguns lemas auxiliares.

Lema 3.1. Existe uma curva de Jordan diferenciável $\Gamma^{+}$, tal que $\Gamma^{+}=\partial R_{0}^{+} \cdot=\Pi_{0}^{+}$A aplicação $S$ leva $R_{0}^{+}$em $R_{0}^{-}$, de forma que o domínio $R_{0}^{-}$é obtido pela reflexão $\rho\left(R_{0}^{+}\right)$, tal que

$$
\rho(v, \tau)=(v,-\tau)
$$

no nos domínios abertos $R_{0}^{+}$e $R_{0}^{-}$temos que

$$
S^{-1}=\rho^{-1} S \rho
$$

Observe que neste caso $\Gamma^{-}=\partial R_{0}^{-}=\Pi_{0}^{-}=\rho\left(\Pi_{0}^{+}\right)=\rho\left(\Gamma^{+}\right)$.

Demonstração. Já sabemos do teorema 4.1 que as curvas de nível $h^{ \pm}(v, \tau)=C>0$ são difeomorfos a círculos. Assim, os cunjuntos $\Pi_{0}^{+}$e $\Pi_{0}^{-}$são ambos difeomorfos a círculos.

Seja $z(t, v, \tau) \operatorname{com}(v, \tau) \in R_{0}^{+}$, das simetrias do problema de Sitnikov, dadas em (17) temos que $z(-t, v,-\tau)$ é uma solução de (13) tal que

$$
z(-(-\tau), v,-\tau)=0, \dot{z}(-(-\tau), v,-\tau)=v
$$

$\operatorname{com} \tau^{\prime}<\tau$. Como $z(-t, v,-\tau) \neq 0$ para $-\tau^{\prime}<t<-\tau$ segue que o ponto $(v,-\tau) \in R_{0}^{-}$.

Como a reflexão $\rho: \Phi \rightarrow \Phi$, tal que $\rho(v, \tau)=(v,-\tau)$ é um difeomorfismo, temos que $R_{0}^{-}=\rho\left(R_{0}^{+}\right)$. Ainda do fato de $\rho$ ser um difeomorfismo, segue que $\rho\left(\Pi_{0}^{+}\right)=\Pi_{0}^{-}$.

Observe que $\rho^{-1}=\rho$, dessa forma

$$
\rho^{-1} S \rho=\rho S \rho
$$

e portanto, temos que

$$
\rho S \rho\left(v^{\prime}, \tau^{\prime}\right)=S\left(v^{\prime},-\tau^{\prime}\right)=\rho(v,-\tau)=(v, \tau) .
$$

Assim concluímos que $S^{-1}=\rho^{-1} S \rho$, isso conclui a prova do lema. 
Lema 3.2. A aplicação $S: R_{0}^{+} \rightarrow R_{0}^{-}$aplica as curvas de nível $h^{+}(v, \tau)=C$ em $h^{-}(v, \tau)=C$ onde $0<C<\Im$.

Demonstração. Da equação (36) da proposição 2.4 sabemos que

$$
h^{-}=h^{+} \circ S \text {. }
$$

Seja $(v, \tau) \in R_{0}^{+}$, sabemos que $h^{+}(v, \tau)=C \operatorname{com} 0<C<\Im$. Seja $\left(v^{\prime}, \tau^{\prime}\right)=S(v, \tau)$, temos que

$$
h^{-}\left(v^{\prime}, \tau^{\prime}\right)=h^{-} \circ S(v, \tau)=h^{+}(v, \tau)=C .
$$

Como $S$ é um difeomorfismo de $R_{0}^{+}$em $R_{0}^{-}$concluímos que a aplicação $S$ leva curvas de nível $h^{+}(v, \tau)=C>0$ em curvas de nível $h^{-}(v, \tau)=C>0$, com $0<C<\Im$.

Lema 3.3. Se a excentricidade $\varepsilon>0$ é suficientemente pequena então $R_{0}^{+} \neq R_{0}^{-}$e $\Pi_{0}^{+}$e $\Pi_{0}^{-}$ intersectam-se transversalmente em $P_{1}=\left(v_{1}, \tau_{1}\right)$, tal que $\tau_{1}=0$ e $\tau_{2}=\pi$. Além disso, as funções $h^{ \pm}$são de classe $C^{1}(\phi \backslash\{O\})$ e o jacobiano

$$
J\left(v_{1}, \tau_{1}\right)=\frac{\partial\left(h^{-}, h^{+}\right)}{\partial(v, \tau)}\left(v_{1}, \tau_{1}\right) \neq 0
$$

Demonstração. A prova deste lema é baseada em cálculos sobre $\partial R_{0}^{+}=\Pi_{0}^{+}$. Sabemos que para um ponto em $\Pi_{0}^{+}$temos

$$
v=z(\tau, \dot{v}, \tau, \varepsilon)
$$

Assim podemos escrever localmente

$$
v=\lambda(\tau, \varepsilon)
$$

Onde $\lambda$ é uma função $C^{\infty}$ em $\tau$ já que

$$
Q(z, t)=\frac{z}{\left(z^{2}+r(t)\right)^{3 / 2}}
$$

é de classe $C^{+\infty}$ na variável $z$.

De acordo com as simetrias dadas em (17) a fronteira $\partial R_{0}^{-}=\Pi_{0}^{-}$e o lema 3.1, para $(v, \tau) \in \Pi_{0}^{-}$escrevemos 


$$
v=\dot{z}(\tau, v,-\tau, \varepsilon)
$$

e dessa forma segue que

$$
v=\lambda(-\tau, \varepsilon)
$$

das equações (57) e (58) vemos que $\Pi_{0}^{+}$e $\Pi_{0}^{-}$coincidem para $\tau=0$. Para que as curvas $\Pi_{0}^{+}$ e $\Pi_{0}^{-}$se intersectem transversalvemte, basta que

$$
\left.\frac{\partial \lambda}{\partial \tau}\right|_{\tau=0} \neq 0
$$

Para isso suficiente que para $\varepsilon>0$ suficientemente pequeno tenhamos.

$$
\left.\frac{\partial^{2} \lambda}{\partial \tau \partial \varepsilon}\right|_{\tau, \varepsilon=0} \neq 0
$$

Observemos que (60) implica (59), basta desenvolver a série de Taylor

$$
\left.\frac{\partial \lambda}{\partial \tau}\right|_{\tau=0}=\frac{\partial \lambda}{\partial \tau}(0,0)+\varepsilon \frac{\partial^{2} \lambda}{\partial \tau \partial \varepsilon}(0,0)+O\left(\varepsilon^{2}\right) .
$$

Como $r(t)$ é analítica, vamos supor que $\lambda$ também é analítica, para podermos usar a sua expressão em série de Taylor. Não perdemos em generalidade ao fazer isso, pois se $\lambda$ não fosse analítica, mas de classe de diferenciabilidade muito alta, poderíamos aproximá-la por polinômios de Taylor, usando a definição de diferenciabilidade. Como $\lambda$ é $C^{\infty}$, o argumento feito é válido.

Com o potencial $U(z)=U(z, t)=\left(z^{2}+r^{2}(t)\right)^{-1 / 2}$ podemos escrever a equação (13) da seguinte forma

$$
\ddot{z}=\frac{\partial U}{\partial z}
$$

A partir disso obtemos uma equação integral para as soluções parabólicas caracterizadas por

$$
\dot{z}(\infty)=0 \text {. }
$$

Multipliquemos a equação (61) por $\dot{z}$ e temos

$$
\ddot{z} \dot{z}=\frac{\partial U}{\partial z} \dot{z}
$$


e integrando de $t$ a $+\infty$ obtemos

$$
\begin{gathered}
\left.\frac{1}{2} \dot{z}^{2}\right|_{t} ^{+}+\infty=\int_{t}^{+\infty} \frac{\partial U}{\partial z} \dot{z} d s \Rightarrow \\
\frac{1}{2} \dot{z}^{2}(t)=-\int_{t}^{+\infty} \frac{\partial U}{\partial z} \dot{z} d s=U(z)
\end{gathered}
$$

Colocando $\varepsilon=0$ em (62) temos que

$$
\frac{1}{2} \dot{z}^{2}=-\int_{t}^{+\infty} \frac{\partial U_{0}}{\partial z} \dot{z} d s=U_{0}(z)
$$

Lembrando que

$$
r(t)=1-\varepsilon \cos (t)+O\left(\varepsilon^{2}\right)
$$

e usando que $U(z)=U(z, t)=\left(z^{2}+r^{2}(t)\right)^{-1 / 2}$, podemos escrever

$$
\left\{\begin{array}{l}
U=U_{0}+\varepsilon U_{1}+O\left(\varepsilon^{2}\right) \\
U_{0}=\left(z^{2}+1\right)^{-1 / 2} \quad, U_{1}=\frac{\cos (t)}{\left(z^{2}+1\right)^{3 / 2}}
\end{array}\right.
$$

Obtemos a equação (63) por expansão em série de Taylor de $U(z)=U(z, \varepsilon)$

$$
\begin{gathered}
U(z, \varepsilon)=U(z, 0)+\varepsilon \frac{\partial U}{\partial \varepsilon}(z, 0)+O\left(\varepsilon^{2}\right) \\
\frac{\partial U}{\partial \varepsilon}=\left.r \frac{\partial r}{\partial \varepsilon} \cdot\left(z^{2}+r^{2}\right)^{-3 / 2}\right|_{(z, \varepsilon=0)}=\frac{\cos (t)}{\left(z^{2}+1\right)^{3 / 2}}
\end{gathered}
$$

Seja $\zeta(t)$ uma solução de (63) satisfazendo

$$
\zeta(0)=0, \dot{\zeta}(0)>0
$$

Então $\zeta(t)$ como construída é parabólica e crescente pois de (63) temos que

$$
\dot{\zeta}(t)=\frac{\sqrt{2}}{(\zeta(t)+1)^{1 / 4}}
$$

Observe que $\dot{\zeta}(0)=\sqrt{2}$ e a solução geral da equação autônoma (63) é escrita na forma $\zeta(t-\tau$. $)$ Dessa forma, podemos escrever. 


$$
z(t, v, \tau, \varepsilon)=\zeta(t-\tau)+\varepsilon z_{1}(t, v, \tau, \varepsilon)+O\left(\varepsilon^{2}\right)
$$

e dessa maneira, segue que

$$
\lambda(\tau, \varepsilon)=\dot{z}(\tau, v, \tau, \varepsilon)=\sqrt{2}+\left.\varepsilon \dot{z}_{1}\right|_{t=\tau}+O\left(\varepsilon^{2}\right)
$$

Desde que $\dot{\zeta}(0)=\sqrt{2}$ a curva $\Pi_{0}^{+}$para $\varepsilon=0$ é uma circunferência de raio $\sqrt{2}$. Essa curva é a primeira aproximação para $\Pi_{0}^{+}$quando $\varepsilon>0$ suficientemente pequeno.

Para calcular $z_{1}$, comparamos os termos em $\varepsilon$ da equação (62)

$$
\dot{z}^{2}=\left(\dot{\zeta}+\varepsilon \dot{z}_{1}+O\left(\varepsilon^{2}\right)\right)^{2}=\dot{\zeta}^{2}+2 \varepsilon \dot{\zeta} \dot{z}_{1}+O\left(\varepsilon^{2}\right)
$$

Disso segue que

$$
\frac{1}{2} \dot{z}^{2}=\frac{\dot{\zeta}}{2}+\varepsilon \dot{\zeta}_{1}+O\left(\varepsilon^{2}\right)
$$

Sabemos de (64) temos

$$
U=U_{0}+\varepsilon U_{1}+O\left(\varepsilon^{2}\right) \Rightarrow \frac{\partial U_{0}}{\partial z}+\varepsilon \frac{\partial U_{1}}{\partial z}+O\left(\varepsilon^{2}\right)
$$

Integrando $\frac{\partial U}{\partial z} \dot{z}$ entre $t$ e $+\infty$, obtemos:

$$
\dot{\zeta} \dot{z}_{1}=-\int_{t}^{+\infty} \frac{\partial U_{1}}{\partial z} \dot{\zeta} d s
$$

Por outro lado sabemos que

$$
\frac{\dot{\zeta}^{2}}{2}=U_{0}(\zeta)=\left(\zeta^{2}+1\right)^{-1 / 2} .
$$

Disso concluímos que

$$
\dot{z}_{1}=-\frac{1}{\sqrt{2}}\left[\frac{\partial U_{1}}{\partial z} \dot{\zeta} d s\right] \cdot\left(\zeta^{2}+1\right)^{-1 / 4}
$$

Coloquemos a condição inicial $z_{1}(\tau)=0$. Consideremos então as seguintes transformações

$$
\left\{\begin{array}{lc}
\omega & =\left(\zeta^{2}+1\right)^{1 / 4} \\
w & =\omega z_{1}
\end{array}\right.
$$


Para $t=\tau$ temos

$$
\dot{w}(\tau)=\omega(\tau) \dot{z}_{1}(\tau)=z_{1}(\tau)=-\frac{1}{\sqrt{2}} \int_{\tau}^{+\infty} \frac{\partial U_{1}}{\partial z} \dot{\zeta} d t, \quad \varepsilon=0
$$

Coloquemos $t=\tau+s$ e usando (64) temos que

$$
\frac{\partial U_{1}}{\partial z}=-\frac{1}{4} \cdot \frac{3}{2} \cdot \frac{2 z\left(z^{2}+1\right)^{1 / 2}}{\left(z^{2}+1\right)^{3}} \cos (t)
$$

Avaliando essa expressão em $\varepsilon=0$ segue que

$$
\left.\frac{\partial U_{1}}{\partial z}\right|_{\varepsilon=0}=-\frac{3}{4} \cdot \frac{\cos t}{\omega^{10}} \cdot \zeta
$$

Com isso concluímos que

$$
\dot{w}(\tau) \frac{1}{\sqrt{2}} \cdot \frac{3}{4} \int_{0}^{+\infty} \cos (\tau+s) \frac{\zeta \dot{\zeta}}{\omega^{10}} d s
$$

Usando a igualdade $z_{1}(\tau)=\omega(\tau)$ quando $\varepsilon=0$. Substituindo em (65) obtemos

$$
\begin{gathered}
\lambda(\tau, \varepsilon)=\sqrt{2}+3 \sqrt{2} \cdot \dot{w}(\tau)+O\left(\varepsilon^{2}\right)= \\
=\sqrt{2}+\frac{3}{8} \cdot \varepsilon(-A \sin \tau+B \cos \tau)+O\left(\varepsilon^{2}\right)
\end{gathered}
$$

Onde

$$
A=\int_{0}^{+\infty} \sin (s) \frac{\zeta \dot{\zeta}}{\omega^{10}}, \quad B=\int_{0}^{+\infty} \cos (s) \frac{\zeta \dot{\zeta}}{\omega^{10}}
$$

A fórmula (68) mostra que $\Pi_{0}^{+}$para $\varepsilon>0$ pequeno é aproximada pela circuferência de centro na origem e raio $\sqrt{2}$. A nossa condição (60) será satisfeita se $A>0$.

De fato

$$
B=\int_{0}^{+\infty}-\cos (s) \frac{\zeta \dot{\zeta}}{\omega^{10}} d s=\int_{\frac{\pi}{2}}^{+\infty} \sin \left(s-\frac{\pi}{2}\right) \frac{\zeta \dot{\zeta}}{\omega^{10}} d\left(s-\frac{\pi}{2}\right)=\int_{\frac{\pi}{2}}^{+\infty} \sin u \frac{\zeta \dot{\zeta}}{\omega^{10}} d u
$$

Para mostrar que $A>0$, usamos o fato de que $\zeta(s)>0, \dot{\zeta}(s)>0, s>0$ e desde que $0<s<\frac{\pi}{2}, \sin s>0$, temos que

$$
\begin{aligned}
A \geq \int_{\frac{\pi}{2}}^{+\infty} \sin (s)\left(\zeta \dot{\zeta} \omega^{-10}\right) d s & =-\left.\cos (s) \frac{\zeta \dot{\zeta}}{\omega^{-10}}\right|_{\frac{\pi}{2}} ^{+\infty}+\cos (s) \frac{d}{d s}\left(\zeta \dot{\zeta} \omega^{-10}\right) d s \\
& =\int_{\frac{\pi}{2}}^{+\infty} \cos s \frac{d}{d s}\left(\zeta \dot{\zeta} \omega^{-10}\right) d s
\end{aligned}
$$




\section{Portanto}

$$
A \geq \int_{\frac{\pi}{2}}^{+\infty} \cos s \frac{d}{d s}\left(\zeta \dot{\zeta} \omega^{-10}\right) d s
$$

Usando a equação diferencial (62)

$$
\frac{\dot{z}^{2}}{2}=-\int_{t}^{+\infty} \frac{\partial U_{0}}{\partial z} \dot{z} d z=U_{0}(z)
$$

para $\zeta$ nós obtemos a relação

$$
\frac{d}{d s}\left(\zeta \dot{\zeta} \omega^{-10}\right)=\ddot{\zeta} \dot{\zeta} \frac{d}{d s}\left(\zeta \omega^{-10}\right)
$$

Por outro lado

$$
\frac{d}{d s}\left(\zeta \dot{\zeta} \omega^{-10}\right)=\ddot{\zeta} \dot{\zeta} \omega^{-10}+\dot{\zeta}^{2}+\left(\frac{\partial}{\partial \zeta}\left(\zeta \omega^{-10}\right)+\right), \quad \dot{\zeta}^{2}=2 \omega^{-2}
$$

Note que $\ddot{\zeta}=\zeta \omega^{-6}$.

Daí obtemos o seguinte

$$
\begin{gathered}
\frac{\partial}{\partial \zeta}\left(\zeta \omega^{-10}\right)=-\zeta \omega^{-6} \zeta \omega^{-10}+2 \omega^{-2}\left(\frac{\partial}{\partial \zeta}\left(\zeta \omega^{-10}\right)\right) \\
\frac{\partial}{\partial \zeta}\left(\zeta \omega^{-10}\right)=\zeta \frac{\partial}{\partial \zeta} \omega^{-10}=\omega^{-10}+\zeta \frac{\left(\zeta^{2}+1\right)^{-5 / 2}}{\partial \zeta}= \\
\omega^{-10}+\frac{5}{2}\left(\zeta^{2}+1\right)^{-7 / 2} \cdot 2 \zeta^{2}=\omega^{-10}-5 \zeta^{2} \omega^{-14}
\end{gathered}
$$

Assim temos que

$$
\frac{\partial}{\partial \zeta}\left(\zeta \omega^{-10}\right)=\omega^{-10}-5 \zeta^{2} \omega^{-14}
$$

Substituindo (71) em (70) obtemos

$$
\begin{gathered}
\frac{d}{d s}\left(\zeta \dot{\zeta} \omega^{-10}\right)=-\zeta^{2} \omega^{-16} 2 \omega^{-2}\left(\omega^{-10}-5 \omega \zeta^{2} \omega^{-14}\right)=-\zeta^{2} \omega^{-16}+2 \omega^{-12}-10 \omega^{-16} \zeta^{2}= \\
=\omega^{-16}\left(11 \zeta^{2}-2 \omega^{4}\right)=\omega^{-16}\left(-9 \zeta^{2}+2\right)=f(\zeta)
\end{gathered}
$$




$$
\frac{d}{d s}\left(\zeta \dot{\zeta} \omega^{-10}\right)=\omega^{-16}\left(-9 \zeta^{2}+2\right)=f(\zeta)
$$

Usaremos o seguinte fato que mostraremos a seguir

$$
\zeta^{2}(s) \geq \zeta^{2}\left(\frac{\pi}{2}\right)>1, \quad s \geq \frac{\pi}{2}
$$

Daí obtemos que $f(\zeta(s))$ é negativo . Da expressão para $\omega$ que é dada em (67), lembrando que $\zeta(s)$ é uma solção do problema dos três corpos quando $\varepsilon=0$, segue que

$$
\lim _{s \rightarrow+\infty} f(\zeta(s))=0
$$

Provemos que $f$ é crescente. Desde que $\dot{\zeta}>0, s>0$ é suficiente provar que

$$
\frac{d f}{d s}>0
$$

De fato

$$
\begin{gathered}
\frac{d f}{d \zeta}=\frac{\partial}{\partial \zeta}\left(\omega^{-16}\left\{-9 \zeta^{2}+2\right\}\right)=\frac{\partial \omega^{-16}}{\partial \zeta} \cdot\left\{-9 \zeta^{2}+2\right\}-18 \omega^{-16} \zeta \\
\frac{\partial \omega^{-16}}{\partial \zeta}=\frac{\partial}{\partial \zeta}\left(\zeta^{2}+1\right)=-8 \zeta\left(\zeta^{2}+1\right)^{-5}=-8 \zeta \omega^{-20}
\end{gathered}
$$

Das duas expressões acima concluímos que

$$
\begin{gathered}
\quad \frac{d f}{d \zeta}=-8 \zeta\left(\zeta^{2}+1\right)^{-5}=-8 \zeta \omega^{-20}\left\{-9 \zeta^{2}+2\right\}-18 \omega^{-16} \zeta= \\
=\zeta \omega^{-20}\left(72 \zeta^{2}-16-18 \omega^{4}\right)=\zeta \omega^{-20}\left(54 \zeta^{2}-34\right)>0, \quad \zeta>1
\end{gathered}
$$

Portanto, desde que provado (73). Obtemos o desejado, a função $f(\zeta(s))$ é crescente em $s$. Substituinto na equação (72) em (69), sabendo (74) e que $f$ é crescente, segue do critério da série alternada que

$$
\int_{\frac{\pi}{2}}^{+\infty} \cos s \frac{d}{d s}\left(\zeta \dot{\zeta} \omega^{-10}\right) d s=\int_{\frac{\pi}{2}}^{+\infty} \cos s f(\zeta(s)) d s
$$

é convergente a um valor positivo. Logo concluímos que $A>0$, como queríamos, disso segue (60) e desta segue (59). 


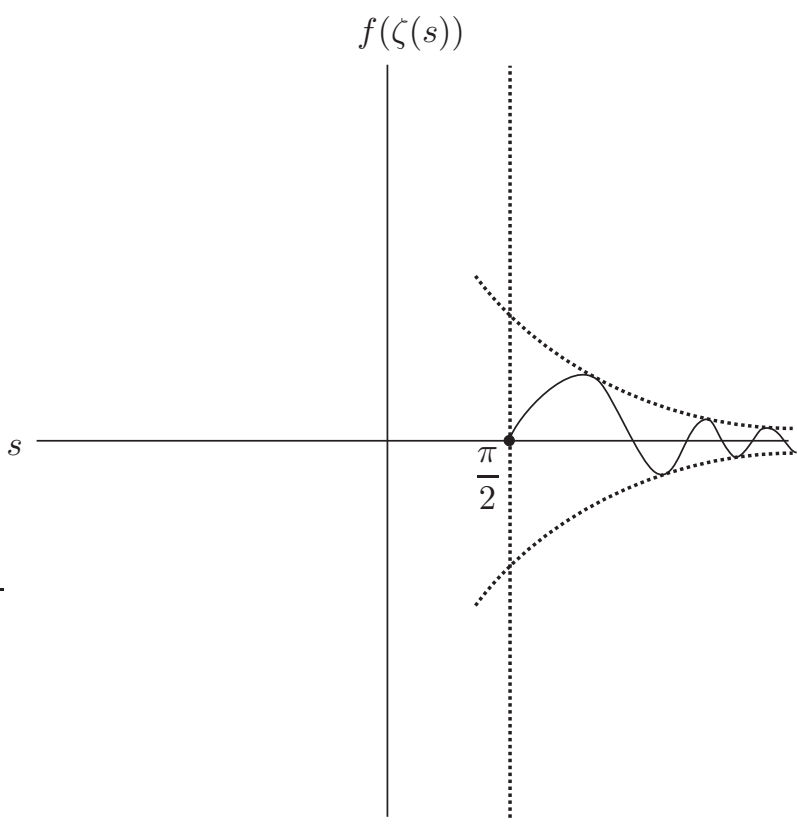

Figura 11: Gráfico de $f(\zeta(s))$.

Para completar o argumento, resta mostrar (73). Usaremos para isso a monotonicidade de $\zeta$. Suponha que $(73)$ seja falsa, isto é $\zeta\left(\frac{\pi}{2}\right) \leq 1$. Do fato de $\zeta$ ser monotonamente crescente temos

$$
\zeta^{2}(s)=\frac{2}{\sqrt{\zeta^{2}+1}} \geq \sqrt{2}>\frac{4}{3}, \quad 0 \leq s \leq \frac{\pi}{2}
$$

Temos a condição inicial $\zeta(0)=0$, usando o teomema do valor médio obtemos:

$$
\left(\zeta\left(\frac{\pi}{2}\right)\right)^{2}=\left(\zeta\left(\frac{\pi}{2}\right)-0\right)\left(\zeta\left(\frac{\pi}{2}\right)-0\right)=(\zeta(\bar{s}))^{2}\left(\frac{\pi}{4}^{2}\right)>\frac{4}{3} \cdot \frac{\pi}{4}^{2}=\frac{\pi}{3}^{2}>1
$$

Mas isso é uma contradição com a hipótese feita.

Para completar a prova do lema resta verificar as propriedades diferenciais de $h^{ \pm}$. Segue do teorema 4.1 do capítulo 4 , que $h^{ \pm}$são de classe $C^{1}(\Phi \backslash\{O\})$ e que os gradientes $\nabla h^{ \pm}$são não nulos sobre $\Phi \backslash\{O\}$. Assim, sobre o ponto $P_{1}=\left(v_{1}, \tau_{1}\right) \in \Pi_{0}^{+} \cap \Pi_{0}^{-}$os vetores gradientes $\nabla h^{ \pm}\left(v_{1}, \tau_{1}\right)$ são não nulos, ortogonais aos vetores tangentes a $\Pi_{0}^{ \pm}$respectivamente sobre o ponto $P_{1}$. Como $\Pi_{0}^{+}$e $\Pi_{0}^{-}$intersectam-se transversalmente, segue que o ângulo entre gradientes $\nabla h^{ \pm}\left(P_{1}\right)$ é o mesmo ângulo módulo $\pi$ que o ângulo formado pelos tangentes a $\Pi_{0}^{+}$e $\Pi_{0}^{-}$sobre o ponto $P_{1}$, o qual é não nulo módulo $\pi$.

Assim $\nabla h^{ \pm}\left(P_{1}\right)$ são ambos não nulos e formam um ângulo não nulo, módulo $\pi$. Desta 
maneira

$$
\left\{\nabla h^{+}\left(P_{1}\right), \nabla h^{-}\left(P_{1}\right)\right\}
$$

é um conjunto linearmente independente, e portanto

$$
J\left(v_{1}, \tau_{1}\right) \neq 0
$$

Isso prova completamente o lema.

Lema 3.4. Seja $\gamma(s):\left(v_{0}=v_{0}(s), \tau_{0}=\tau_{0}(s)\right)$, com $0 \leq s \leq 1$ um arco $C^{1}$ tal que $\gamma$ satisafça

$$
\gamma(0)=q \in R_{0}^{+}, \lim _{s \rightarrow 1} \gamma(s)=p \in \Pi_{0}^{+},
$$

de forma que $\gamma(s) \in R_{0}^{+}$para $0 \leq s<1$. Então a curva $S(\gamma(s))=\left(v_{1}(s), \tau_{1}(s)\right)$ aproxima-se de $\Pi_{0}^{-}$, espiralando-se, isto é $\tau_{1}(t) \rightarrow+\infty$ quando $t \rightarrow 1$.

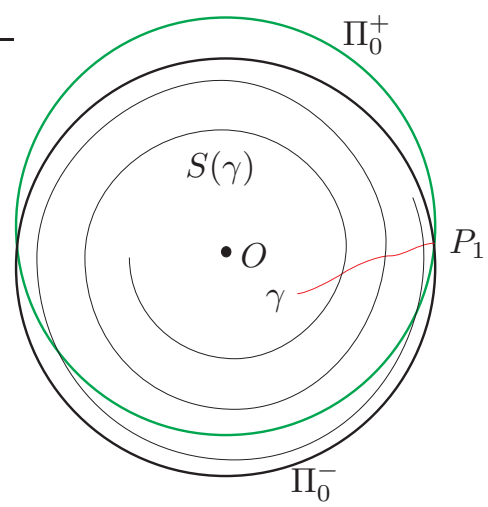

Demonstração. De fato, da continuidade de $h^{+}$e $\gamma$ temos que

$$
\lim _{s \rightarrow 1} h^{+}(v(s), \tau(s))=\Im
$$

Do lema 3.2, segue que

$$
\lim _{s \rightarrow 1} S(\gamma(s))=\Im
$$

Desta maneira, temos que $\left(v_{1}(s), \tau_{1}(s)\right)$ está se aproximando de $\Pi_{0}^{-}$. Como $T^{+}$é contínua em $R_{0}^{+}$temos que

$$
\lim _{s \rightarrow 1} T^{+}(\gamma(s))=+\infty \rightarrow \lim _{s \rightarrow 1} \tau_{1}(s)=+\infty
$$


pois $\tau_{1}(s)>T^{+}(v(s), \tau(s))$ para $0 \leq s<1$. Isso prova completamente o lema.

O lema acima é verdadeira se em vez de $\Pi_{0}^{+}$considerarmos $\Pi_{0}^{-}$e trocarmos $S$ por $S^{-1}$ e a demonstração segue diretamente da expressão:

$$
S^{-1}=\rho^{-1} S \rho
$$

que foi obtida do lema 3.1. Observe que dos lemas 3.2 e 3.4 segue que a aplicação $S$ aplica o anel $0 \leq C \leq h^{+}(v, \tau)<\Im$ sobre o anel $0 \leq C \leq h^{-}(v, \tau)<\Im$.

Teorema 3.5 (Conjugação da Dinâmica $S$ com o shift de $n$ Símbolos). Seja $n$ um número natural, existe uma região $R \subset R_{0}^{+} \cap R_{0}^{-}$e um subconjunto $I_{n} \subset R$ tal que a dinâmica $S$ restrita a $I_{n}$ é conjugada ao shift $\sigma: \Sigma_{A} \rightarrow \Sigma_{A}$, onde $A=\{1, \ldots n\}$.

Demonstração. Seja $\varepsilon>0$ suficientemente pequeno, pelos lemas 3.3 e 3.4 fixemos uma região $R$ dada abaixo:

$$
R^{\prime}=\left\{(v, \tau) \in \Phi \mid \Im-\varepsilon \leq h^{+}(v, \tau), h^{-}(v, \tau) \leq \Im\right\}
$$

Note que $R^{\prime}$ é a interseção de dois aneis

$$
A_{1}: \Im-\varepsilon \leq h^{+}(v, \tau)<\Im
$$

e

$$
A_{2}: \Im-\varepsilon \leq h^{-}(v, \tau)<\Im
$$

Podemos folhear $R^{\prime}$ pelas curvas de nível $h^{-}(v, \tau)=C$ com $\Im-\varepsilon \leq C \leq \Im$, as quais intersectam $\Pi_{0}^{+}$, sendo assim, pelo lema 3.4, a imagem de $R$ por $S$ tem a seguinte forma.

Podemos escolher um então um subconjunto $R \subset R^{\prime}$ tal que a $R \cap S(R)$ é decomposta em infinitas componentes conexas, as quais se aproximam de $\Pi_{0}^{+} \cap R$. Chamamos estas componentes de

$$
V_{1}, V_{2}, \ldots V_{n}, \ldots
$$




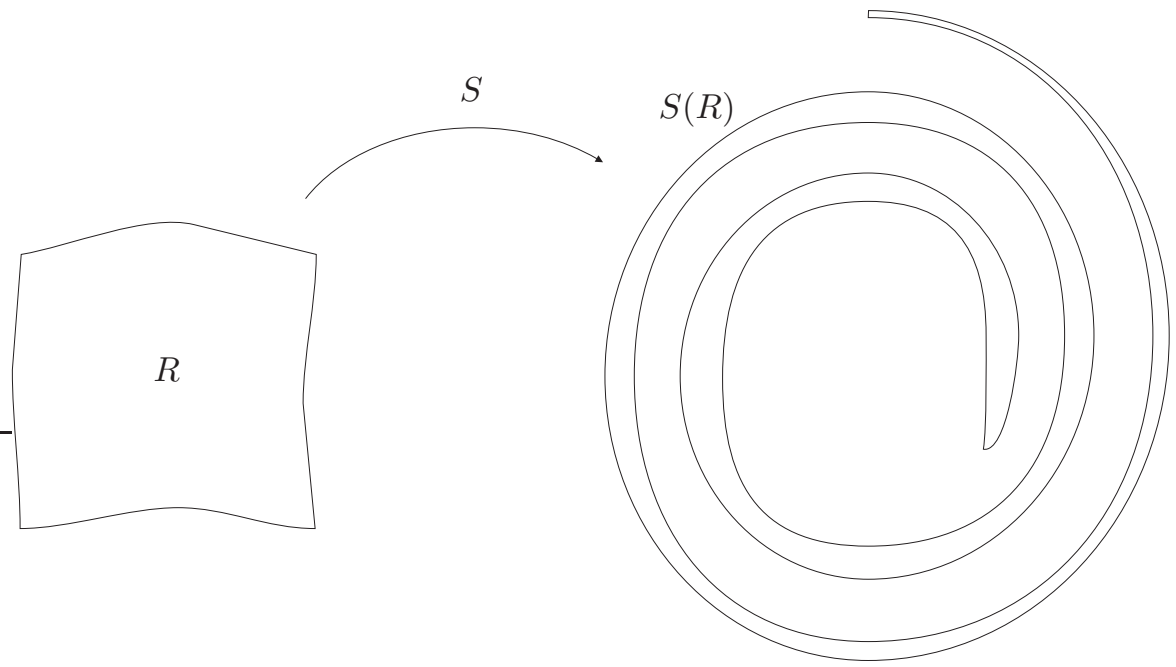

Figura 12: Imagem de $R$ via aplicação $S$.

Também podemos folhear $R^{\prime}$ pelas curvas de nível $h^{+}(v, \tau)=C$ com $\Im-\varepsilon \leq C \leq \Im$, as quais intersectam $\Pi_{0}^{-}$, com isso $R \cap S^{-1}(R)$ é decomposta em infinitas componentes conexas, as quais se aproximam de $\Pi_{0}^{-} \cap R$. Chamamos estas componentes de

$$
U_{1}, U_{2}, \ldots U_{n}, \ldots
$$

Observe pela definição que, como $S$ é um difeomorfismo temos :

$$
S\left(V_{i}\right)=U_{i}, i \in \mathbb{N} \text {. }
$$

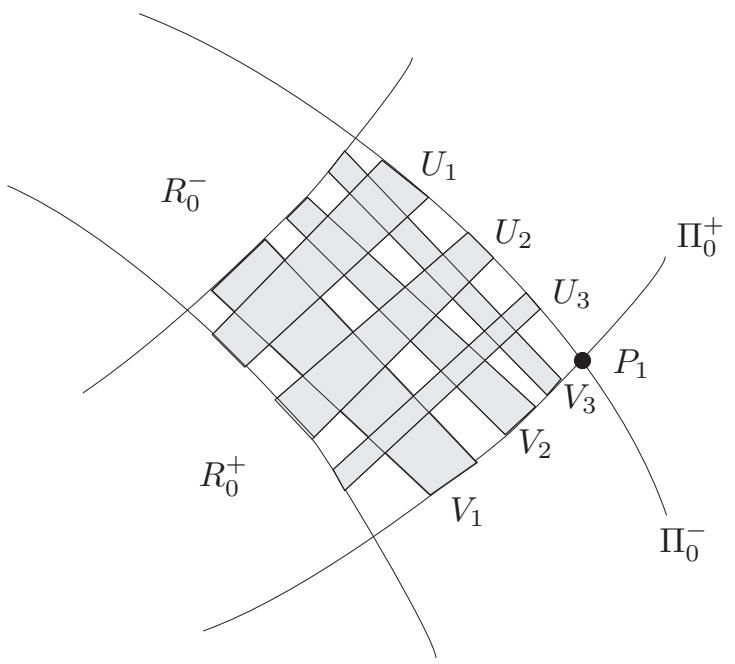


A aplicação $S$ é de classe $C^{1}$ como podemos observar das combinações dos seguintes resultados: proposição 4.1, proposição 4.2 e corolários 4.1 e 4.2 .

Introduzimos agora a mudança de coordenadas (112)

$$
\xi=h^{-}(v, \tau), \eta=h^{+}(v, \tau)
$$

Essa mudança de coordenadas é regular sobre o ponto $P_{1}$, isto segue dos teoremas 4.1, e 4.2. Se considerarmos um plano $\mathbb{R}^{2}$ nas coordenadas $(\xi, \eta)$ vemos que essa mudança de coordenadas leva as curvas de nível $h^{-}(v, \tau)=C>0$ em linhas verticais

$$
\xi=C,
$$

e leva as curvas de nível $h^{+}(v, \tau)=C$ em linhas horizontais

$$
\eta=C
$$

Nas coordenadas $(\xi, \eta)$ a região $R^{\prime}$ se transforma num quadrado

$$
Q^{\prime}=[\Im-\varepsilon, \Im] \times[\Im-\varepsilon, \Im]
$$

A aplicação $S$ nas coordenadas $\xi$ e $\eta$ tem o aspecto dado em (113).

$$
s(\xi, \eta)=(\eta, f(\xi, \eta))
$$

Como a mundança de coordenadas (112) é de classe $C^{1}(\phi \backslash\{O\})$, a transformação $S$ dada em (113) é de classe $C^{1}\left(\mathbb{R}^{2}\{O\}\right)$.

Podemos supor que $\varepsilon>0$ seja suficientemente pequeno tal que o ângulo entre os vetores tangentes unitários em pontos das fronteiras das componentes $V_{i}, i \in \mathbb{N}$, e o respectivo ponto mais próximo em $\Pi_{0}^{+}$seja muito próximo de 0 . O mesmo podemos falar das componentes $U_{i}$ com relação a $\Pi_{0}^{-}$. Podemos ainda supor que a fronteiras de $R$ satisfaça as mesmas condições impostas sobre as fronteiras de $U_{i}$ e $V_{i}$ para $i \in \mathbb{N}$. Este tipo de afirmação pode ser feita pois tais fronteiras são obtidas como curvas que se espiralam em $\Pi_{0}^{+}$e $\Pi_{0}^{-}$respectivamente, conforme o lema 3.4.

Denotemos por $Q$, a imagem de $R$ via transformação (112). A transformação dada em (112) é de classe $C^{1}$ numa vizinhança de $P_{1}$, as componentes $V_{1}, \ldots, V_{n}, \ldots$, são levadas em faixas verticais em $Q$, por simplicidade, manteremos a mesma nomenclatura. Analogamente, 
as componentes $U_{1}, \ldots, U_{n}, \ldots$, são levadas em faixas horizontais em $Q$, essas faixas também chamaremos de $U_{i}, i \in \mathbb{N}$.

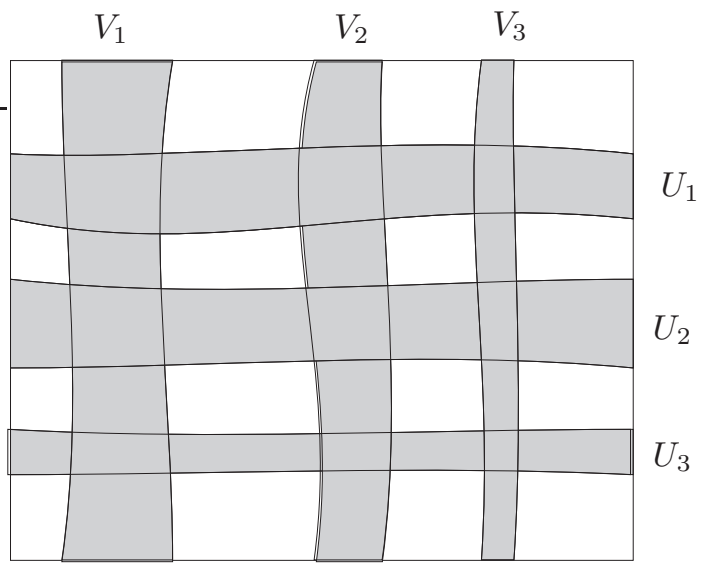

Sabemos da proposição 4.4 que, a aplicação $S$ nas coordenadas $(\xi, \eta)$ é conjugado com a aplicação $S$ nas coordenadas $(v, \tau)$. Como existe uma conjugação dada pela mudança de coordenadas $(112)$, basta que provemos o resultado em $(\xi, \eta)$. De agora em diante, trabalharemos apenas nas coordenadas $(\xi, \eta)$.

Temos que

$$
S\left(V_{i}\right)=U_{i}, i \in \mathbb{N} \text {. }
$$

De acordo com a proposição 4.4 a matriz jacobiana de $d S$ numa vizinhança de $(\Im, \Im)$ ou equivalentemente, numa vizinhança de $P_{1}$ é dada por

$$
d S(\xi, \eta)=\left(\begin{array}{cc}
0 & 1 \\
-O(1) & \frac{\alpha J\left(v^{\prime}, \tau^{\prime}\right)}{v^{2} Q_{0}\left(X^{+}(v, \tau)\right)}+1-O(1)
\end{array}\right)
$$

e conforme a proposição 4.5 o diferencial $d S$ em $Q$ satisfaz as condições dos teoremas 1.2, 1.3 e 1.4 para $\mu=\frac{1}{4}$, desde que $\varepsilon>0$ seja suficientemente pequeno. E vamos construir sobre $R$ uma região dada como abaixo 


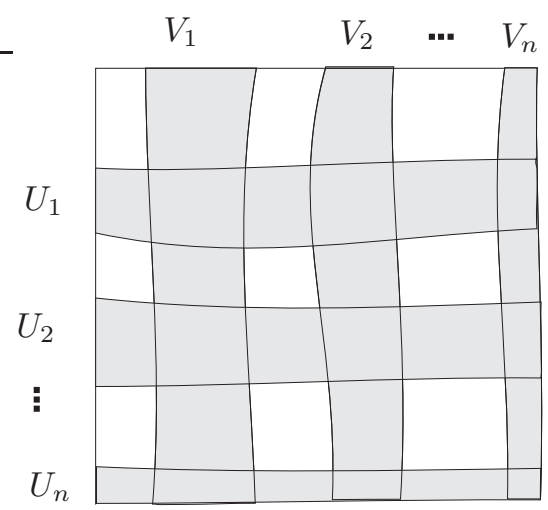

Que é limitada pelas fronteiras das faixas $V_{n}$ e $U_{n}$. Para simplificar, chamemos esta nova região também de $Q$ e a mesma satisfaz que

$$
\begin{gathered}
S(Q) \cap Q=V_{1} \cup \ldots \cup V_{n} \\
S^{-1}(Q) \cap Q=U_{1} \cup \ldots \cup U_{n}
\end{gathered}
$$

e além disso segue que $S\left(V_{i}\right)=U_{i}, \quad i=1, \ldots, n$.

A aplicação $S$, restrita a $Q$, satisfeitaz as condições dos teoremas 1.2, 1.3 e 1.4, disso segue o teorema 1.1, donde concluímos a existência de um conjunto hiperbólico invariante $I_{n} \subset Q$ tal que $\left.S\right|_{I_{n}}$ é conjugada ao shift em $\Sigma_{A}, A=\{1, \ldots, n\}$.

Do teorema de conjugação com o shift de n símbolos, seguem diretamente os seguites corolários:

Corolário 3.1. Existem condições iniciais $(v, \tau)$ tal que a solução correspondente

$$
z(t, v, \tau)
$$

é periódica.

Corolário 3.2. O ponto $P_{1}$ pode ser aproximado por ponto $p_{1}, p_{2}, \ldots, p_{n}, \ldots$ tais que as soluções

$$
z\left(t, v_{i}, \tau_{i}\right)
$$

com condições iniciais $p_{i}=\left(v_{i}, \tau_{i}\right)$ são todas órbitas periódicas.

Corolário 3.3. Todos os conjuntos $R_{n}^{ \pm}, \Pi_{n}^{ \pm}$, e $H_{n}^{ \pm}$, definidos em (37) são não vazios para todo $n \geq 0$. 


\section{Propriedades Diferenciais das Aplicações}

Nessa secção faremos um estudo detalhado das propriedades diferenciais vistas nas secções anteriores, $h^{+}, h^{-}$e $S$. Assumiremos daqui em diante que $Q(z, t)$ satisfaça condições mais restritas do que as descritas em 4.1

$\left.3^{\circ \circ}\right) Q(z, t) \geq q(z), \forall z>0, \quad \forall t$

A condição $4^{\mathrm{o}}$ também permanece no caso $\Im=\int_{0}^{+\infty} Q_{0}(z) d z<+\infty$, e caso contrário nós trocaremos esta por

$\left.4^{\mathrm{oo}}\right)$ A derivada $\frac{\partial^{2} Q}{\partial t^{2}}(z, t)$ é contínua para todo $z>0$ e para todo $t$, e satifaz

$$
\left|\frac{\partial^{2} Q}{\partial t^{2}}(z, t)\right| \leq \psi_{1}(z) \text { e } \int_{0}^{+\infty} z \psi_{1}(z)<\infty
$$

Finalmente imporemos as seguintes condições

$\left.5^{\circ}\right)$ Existe $z^{*}>0$ tal que a função

$$
\xi(z)=\sup _{z^{*} \leq y \leq z} \frac{\psi(z)}{q(y) q(z)}\left\{\begin{array}{c}
\text { é limitada para } z \leq z^{*} \text { se } \Im<+\infty \\
=o\left(\frac{1}{z}\right) \text { quando } z \rightarrow+\infty \text { Se } \Im=+\infty
\end{array}\right.
$$

$\left.6^{\circ}\right) \frac{\partial Q}{\partial z}(z, t) \leq 0, z \leq z^{*}$

Além disso analizaremos o problema somente no caso em que $\Im$ é finito.

\subsection{Propriedades Diferenciais das Aplicações $Z^{ \pm}, T^{ \pm}, h^{ \pm}$}

Lema 4.1. Se a condição $4^{o o}$ é satisfeita, então $4^{\circ}$ é satisfeita:

$$
\left|Q_{t}^{\prime}(z, t)\right| \leq \psi(z)=2 \pi \psi_{1}(z)
$$

onde

$$
\int_{0}^{+\infty} z \psi(z) d z<+\infty \text { e } \int_{x}^{+\infty} \psi(z) d z=o\left(\frac{1}{z}\right) \text { quando } x \rightarrow+\infty
$$


Demonstração. Temos que $Q(z, t)$ é periódica em $t$ e dessa forma, a média de sua derivada com relação ao tempo é zero, de fato:

$$
\frac{1}{2 \pi} \int_{0}^{2 \pi} Q_{t}^{\prime}(z, s) d s=\frac{Q(z, 2 \pi)-Q(z, 0)}{2 \pi}=0 .
$$

Daí segue que

$$
Q_{t}^{\prime}(z, t)=Q(z, t)-\frac{1}{2 \pi} \int_{0}^{2 \pi} Q_{t}^{\prime}(z, s) d s
$$

e aplicando o raciocínio do lema 2.1 temos que

$$
\left|Q_{t}^{\prime}(z, t)\right|=\left|Q_{t}^{\prime}(z, t)-Q_{t}^{\prime}(z, s)\right| \leq\left|Q_{t}^{\prime \prime}(z, \bar{t})(t-\bar{t})\right| \leq 2 \pi\left|Q_{t}^{\prime \prime}(z, t)\right|
$$

com isso e pela condição $4^{\text {oo }}$ temos que

$$
\left|Q_{t}^{\prime}(z, t)\right| \leq 2 \pi \psi_{1}(z)=\psi(z)
$$

Observemos agora que

$$
\int_{z}^{+\infty} \psi(y) d y \leq z \int_{z}^{+\infty} \psi(y) d y \text { quando, } z \rightarrow+\infty
$$

Assim, segue que

$$
\begin{gathered}
z \int_{z}^{+\infty} \psi(y) d y=\int_{z}^{+\infty} z \psi(y) d y \leq \int_{z}^{+\infty} y \psi(y) d y \Rightarrow \\
\int_{z}^{+\infty} \psi(y) d y \leq \frac{1}{z} \int_{z}^{+\infty} y \psi(y) d y, \text { e } \int_{z}^{+\infty} y \psi(y) d y \rightarrow 0 z \text { quando } z \rightarrow+\infty \Rightarrow \\
\frac{\int_{z}^{+\infty} \psi(y) d y}{\frac{1}{z}} \leq \int_{z}^{+\infty} y \psi(y) d y \rightarrow 0 \text { quando } z \rightarrow+\infty \Rightarrow \int_{z}^{+\infty} \psi(y) d y=o(1 / z)
\end{gathered}
$$

Proposição 4.1. As funções $Z^{ \pm}(v, \tau)$ e $T^{ \pm}(v, \tau)$ são de classe $C^{1}\left(R_{0}^{ \pm}\right)$. Se $\tau$ é limitado inferiormente, então

$$
\lim _{Z^{ \pm} \rightarrow+\infty} T^{ \pm}(v, \tau)=+\infty
$$


Demonstração. Seja

$$
M=\sup _{[0,2 \pi] \times\left[0, z^{*}\right]} Q(z, t) .
$$

Consideremos $z \geq z^{*}$, sabemos que para cada $t$ fixado $Q(z, t)$ é decrescente em $z$ para $z \geq z^{*}$ daí segue que

$$
Q(z, t) \leq M \quad \forall t \in \mathbb{R}, \quad \forall z \in \mathbb{R}
$$

Sendo assim $Q(z, t)$ é limitado superiormente por $M$. Daí pela proposição 2.2, a equação (75) é verdadeira.

Agora passaremos à prova de que $Z$ e $T$ são funções de classe $C^{1}\left(R_{0}\right)$. As funções $Z$ e $T$ satisfazem as seguintes equações:

$$
z\left(T^{ \pm}, v, \tau\right) \mp Z^{ \pm}=0, \quad \dot{z}\left(T^{ \pm}, v, \tau\right)=0
$$

Podemos considerar duas aplicações $F: \mathbb{R}^{4} \rightarrow \mathbb{R}^{2}$ dada por

$$
F^{ \pm}\left(v, \tau, T^{ \pm}, Z^{ \pm}\right)=\left(z\left(T^{ \pm}, v, \tau\right) \mp Z, \dot{z}\left(T^{ \pm}, v, \tau\right)\right)
$$

As aplicações $F^{ \pm}$acima, ela teoria das equações diferenciais ordinárias, é de classe $C^{1}$ em suas entradas. Calculando o Jacobiano de $F^{ \pm}$com relação às variáveis $Z^{ \pm}$e $T^{ \pm}$temos que

$$
\left|\begin{array}{cc}
\mp 1 & \dot{z}\left(T^{ \pm}, v, \tau\right) \\
0 & \ddot{z}(T, v, \tau)
\end{array}\right|=\left|\begin{array}{cc}
-1 & \dot{z}\left(T^{ \pm}, v, \tau\right) \\
0 & -Q\left(Z^{ \pm}, T^{ \pm}\right)
\end{array}\right|= \pm Q\left(Z^{ \pm}, T^{ \pm}\right) \neq 0 \text { por } 3^{\text {oo }}
$$

Dessa forma, pelo teorema da função implícita $Z^{ \pm}$e $T^{ \pm}$são de classe $C^{1}\left(R_{0}\right)$.

Se substitirmos ambos os símbolos, $\frac{\partial}{\partial v} \mathrm{e} \frac{\partial}{\partial \tau}$, por $\delta$, a partir das equações (76) temos que

$$
\delta Z^{ \pm}= \pm \delta z(T, v, \tau) ; \quad \ddot{z} \delta T^{ \pm}+\delta \dot{z}=0 \Rightarrow \delta T^{ \pm}=\frac{\delta \dot{z}}{Q\left(Z^{ \pm}, T^{ \pm}\right)}
$$

Lembremos que sobre $\Phi$ identificamos os valores de $\tau$ que diferenciam por múltiplos inteiros de $2 \pi$. A parir das equações (16) temos que $T^{ \pm}(v, \tau+2 \pi)=T^{ \pm}(v, \tau)+2 \pi$, logo também podemos considerar $T^{ \pm}(v, \tau)$ em coordenadas angulares. Seja o anel

$$
K^{ \pm}=\left\{\left(h^{ \pm}, T^{ \pm}\right) \mid 0<h^{ \pm}<\Im, T^{ \pm} \bmod 2 \pi\right\}
$$


Proposição 4.2. A aplicação $P^{+}: R_{0}^{+} \rightarrow K^{+}$definida por

$$
P^{+}(v, \tau)=\left(h^{+}(v, \tau) ; T^{+}(v, \tau) \bmod 2 \pi\right)
$$

é um difeomorfismo. O mesmo vale para o análogo $P^{-}$.

Demonstração. Nesta demosntração omitiremos o símbolo \pm bem como em outras demonstrações que ambos os casos são análogos.

Na região $R_{0}$ temos que

$$
h(v, \tau)=\int_{0}^{Z(v, \tau)} Q_{0}(z) d z
$$

onde já sabemos que

$$
Q_{0}(z)=\frac{1}{2 \pi} \int_{0}^{2 \pi} Q(z, t) d t \geq q(z)>0
$$

por $3^{\text {oo }}$. Então os valores de $h$ no intervalo $(0, \Im)$ estão em correspondência biunívoca com os valores de $Z(v, \tau)$ em $(0,+\infty)$.

Se $\left(v_{1}, \tau_{1}\right) \neq\left(v_{2}, \tau_{2}\right)$ implica que $Z\left(v_{1}, \tau_{1}\right) \neq Z\left(v_{2}, \tau_{2}\right)$ ou $T\left(v_{1}, \tau_{1}\right) \neq T\left(v_{2}, \tau_{2}\right)$.

De fato, como estamos considerando $\tau$ e $T(v, \tau)$ como coordenadas angulares idenficadas módulo $2 \pi$, se tivéssemos

$$
\begin{gathered}
T\left(v_{1}, \tau_{1}\right)=T\left(v_{2}, \tau_{2}\right)=T \\
z\left(T\left(v_{1}, \tau_{1}\right), v_{1}, \tau_{1}\right)=Z\left(v_{1}, \tau_{1}\right)=Z\left(v_{2}, \tau_{2}\right)=z\left(T\left(v_{2}, \tau_{2}\right), v_{2}, \tau_{2}\right)
\end{gathered}
$$

Como $\dot{z}\left(T\left(v_{i}, \tau_{i}\right), v_{i}, \tau_{i}\right)=0$ para $i=1,2$ teríamos então com isto e ambas as igualdades acima juntamente com o teorema de existência e unicidade de soluções de equações diferenciais ordinárias temos que:

$$
z\left(t, v_{1}, \tau_{1}\right) \equiv z\left(t, v_{2}, \tau_{2}\right) \equiv z(t, 0, T) \Rightarrow\left(v_{1}, \tau_{1}\right)=\left(v_{2}, \tau_{2}\right)
$$

mas isto é absurdo, $\operatorname{logo}\left(v_{1}, \tau_{1}\right) \neq\left(v_{2}, \tau_{2}\right)$ implica que $Z\left(v_{1}, \tau_{1}\right) \neq Z\left(v_{2}, \tau_{2}\right)$ ou $T\left(v_{1}, \tau_{1}\right) \neq$ $T\left(v_{2}, \tau_{2}\right)$. Assim, $P$ é uma função injetora.

Mostremos agora que $P$ é sobrejetora. Seja o par $\left(h_{0}, T_{0}\right) \in K$. Seja $Z_{0} \in(0, \Im)$ tal que 


$$
\int_{0}^{Z_{0}} Q_{0}(z) d z=h_{0}
$$

Consideremos então a solução $z(t)$ que satisfaz as condições iniciais

$$
z\left(T_{0}\right)=Z_{0}, \quad \dot{z}\left(T_{0}\right)=0
$$

Sendo assim, existe $(v, \tau)$ conveniente tal que

$$
z(t, v, \tau)=z(t)
$$

assim, temos que

$$
P(v, \tau)=\left(h_{0}, T_{0}\right)
$$

como queríamos.

Para mostrar que $P: R_{0} \rightarrow K$ é um difeomorfismo, basta mostrar que é um difeomorfismo local, pois já sabemos que esta é bijetora.

Calculemos o Jacobiano

$$
\frac{\partial(h, T)}{\partial(v, \tau)}=\left|\begin{array}{cc}
Q_{0}(Z) \frac{\partial z}{\partial v} & Q_{0}(Z) \frac{\partial z}{\partial \tau} \\
\frac{\partial \dot{z}}{\partial v} \frac{1}{Q(Z, T)} & \frac{\partial \dot{z}}{\partial v} \frac{1}{Q(Z, T)}
\end{array}\right|=\frac{Q_{0}(Z)}{Q(Z, T)} \frac{\partial(z, \dot{z})}{\partial(v, \tau)}=v \frac{Q_{0}(Z)}{Q(Z, T)}>0
$$

Notemos que

$$
\frac{\partial(z, \dot{z})}{\partial(v, \tau)}=v
$$

independentemente do tempo $t$. De fato

$$
\frac{\partial(z, \dot{z})}{\partial(v, \tau)}=\left|\begin{array}{cc}
z_{v}(t, v, \tau) & z_{\tau}(t, v, \tau) \\
\dot{z}_{v}(t, v, \tau) & \dot{z}_{\tau}(t, v, \tau)
\end{array}\right|=z_{v}(t, v, \tau) \dot{z}_{\tau}(t, v, \tau)-z_{\tau}(t, v, \tau) \dot{z}_{v}(t, v, \tau)
$$

usando o teorema de Schwarz e derivando o lado direito da igualdade acima com respeito a $t$ obtmos 


$$
\frac{d}{d t}\left[z_{v} \dot{z}_{\tau}-z_{\tau} \dot{z}_{v}\right]=\dot{z}_{v} \dot{z}_{\tau}+\ddot{z}_{\tau} z_{v}-\left(\dot{z}_{\tau} \dot{z}_{v}+\ddot{z}_{v} z_{\tau}\right)=0
$$

Observemos as identidades

$$
\begin{aligned}
& z(\tau, v, \tau) \equiv 0 \\
& \dot{z}(\tau, v, \tau) \equiv v
\end{aligned}
$$

Da identidade (78) temos que

$$
z_{\tau}(\tau, v, \tau)+v=0, \quad z_{v}(\tau, v, \tau)=0
$$

segue da identidade (79) que

$$
\dot{z}_{v}(\tau, v, \tau)=\frac{d v}{d v}=1, \quad \dot{z}_{\tau}(\tau, v, \tau)=\frac{d v}{d \tau}=0
$$

Assim, calculando $\frac{\partial(z, \dot{z})}{\partial(v, \tau)}$ em $t=\tau$ obtemos

$$
\frac{\partial(z, \dot{z})}{\partial(v, \tau)}=\left|\begin{array}{cc}
z_{v}(t, v, \tau) & z_{\tau}(t, v, \tau) \\
\dot{z}_{v}(t, v, \tau) & \dot{z}_{\tau}(t, v, \tau)
\end{array}\right|=\left|\begin{array}{cc}
0 & -v \\
1 & 0
\end{array}\right|=v>0
$$

Logo, pelo teorema da função inversa $P$ é um difeomorfismo.

Corolário 4.1. A função $h^{+}$é de classe $C^{1}\left(R_{0}^{+}\right)$e o gradiente $\nabla h^{+} \neq 0$ e as curvas de nível de $h^{+}(v, \tau)=c, 0<c<\Im$ são difeomorfos a círculos.

Demonstração. De fato, se $P \in C^{1}\left(R_{0}\right)$ então, como estamos em dimensão finita, cada função coordenada é também de classe $C^{1}\left(R_{0}\right)$. Também temos que $\nabla h \neq 0$, em $R_{0}$ pois se assim o fosse teríamos $\frac{\partial(h, T)}{\partial(v, \tau)}=0$.

Seja $0<C<\Im$ e considere agora a curva de nível

$$
\gamma=\{(v, \tau) \mid h(v, \tau)=C\}
$$

Temos que $\gamma$ é difeomorfa a $P(\gamma)$

$$
P(\gamma)=\{C, T(v, \tau) \bmod 2 \pi\}
$$


Como $P$ é sobretora, $T(v, \tau) \bmod 2 \pi$ percorre todos os valores dos ângulos em $[0,2 \pi)$. Assim $P(\gamma)$ é um círculo. Isto completa a demonstração.

Corolário 4.2. A aplicação $S: R_{0}^{+} \rightarrow R_{0}^{-}$satisfaz

$$
S=\left(P^{-}\right)^{-1} \circ P^{+}
$$

Demonstração.

$P^{+}(v, \tau)=\left(h^{+}(v, \tau), T^{+}(v, \tau)\right)=\left(h^{-}\left(v^{\prime}, \tau^{\prime}\right), T^{-}\left(v^{\prime}, \tau^{\prime}\right)\right)=\left(h^{-} \circ S(v, \tau), T^{-} \circ S(v, \tau)=P^{-} \circ S(v, \tau) \Rightarrow\right.$

$$
\Rightarrow S=\left(P^{-}\right)^{-1} \circ P^{+}
$$

\section{Lema 4.2.}

1) $S e 0 \leq y \leq Z^{ \pm}(v, \tau)$, então $V(y, v, \tau) \geq\left[\int_{y}^{Z^{+}(v, \tau)} 2 q(\sigma) d \sigma\right]^{1 / 2}$

2) $S e z^{*} \leq z_{0}, z_{1} \leq z_{2} \leq Z^{ \pm}(v, \tau)$, onde $z^{*}$ é definido em $5^{\circ}$, então

$$
\int_{z_{0}}^{z_{2}} \psi(s) \int_{z_{1}}^{s} \frac{d \sigma d s}{V^{3}(\sigma, v, \tau)} \leq\left[\max _{z_{0} \leq s \leq z_{1}} \xi(s)\right]\left[2 \int_{z_{0}}^{Z^{ \pm}(v, \tau)} q(\sigma) d \sigma\right]
$$

Demonstração. Pela equação (29) temos que

$$
\frac{\partial}{\partial y}\left(\frac{V(y, v, \tau)^{2}}{2}\right)=-Q(y, t(y, v, \tau))
$$

e assim segue que

$$
\frac{V^{2}}{2}-\int_{y}^{Z(v, \tau)} Q(\sigma, t) d \sigma=\frac{V}{2}^{2}+\int_{y}^{Z(v, \tau)} \frac{\partial}{\partial y}\left(\frac{V^{2}}{2}\right) d \sigma=\lim _{\sigma \rightarrow Z(v, \tau)} \frac{V(\sigma, v, \tau)^{2}}{2}=0
$$

logo temos que

$$
\frac{V(y, v, \tau)^{2}}{2}=\int_{y}^{Z(v, \tau)} Q(\sigma, t) d \sigma \geq \int_{y}^{Z(v, \tau)} q(\sigma) d \sigma \Rightarrow V \geq\left[2 \int_{y}^{Z(v, \tau)} q(\sigma) d \sigma\right]^{1 / 2}
$$

Temos assim provada a primeira parte do lema.

Seja agora $z_{1} \geq z^{*}$, conforme a notação introduzida em $5^{\circ}$. Aplicando a fórmula da primeira parte deste lema e usando $5^{\circ}$ obtemos

$$
\int_{z_{0}}^{z_{1}} \psi(z) \int_{z_{1}}^{s} \frac{d \sigma d s}{V^{3}(\sigma)} \leq \int_{z_{0}}^{z_{2}} \xi(s) q(s) q(\sigma) \int_{z_{1}}^{s} \frac{d \sigma d s}{\left[2 \int_{\sigma}^{s} q(\xi) d \xi\right]^{3 / 2}} \leq
$$




$$
\begin{gathered}
\leq \int_{z_{0}}^{z_{2}} \xi(s) q(s) \int_{z_{1}}^{s} \frac{q(\sigma) d \sigma d s}{\left[2 \int_{\sigma}^{Z(v, \tau)} q(\xi) d \xi\right]^{3 / 2}}=\left.\int_{z_{0}}^{z_{2}} \xi(s) q(s)\left[2 \int_{\sigma}^{Z(v, \tau)} q(\xi) d \xi\right]^{-1 / 2}\right|_{z_{1}} ^{s} d s= \\
=\int_{z_{0}}^{z_{2}} \xi(s) q(s)\left[\frac{1}{\left[2 \int_{s}^{Z(v, \tau)} q(\xi) d \xi\right]^{1 / 2}}-\frac{1}{\left[2 \int_{z_{1}}^{Z(v, \tau)} q(\xi) d \xi\right]^{1 / 2}}\right] d s \leq \\
\leq\left[\max _{z_{0} \leq s \leq z_{1}} \xi(s)\right] \int_{z_{0}}^{z_{2}} \frac{q(s)}{\left[2 \int_{s}^{Z(v, \tau)} q(\sigma) d \sigma\right]^{1 / 2}}= \\
=\left[\max _{z_{0} \geq s \geq z_{1}} \xi(s)\right]\left[2 \int_{z_{0}}^{z_{2}} q(\sigma) d \sigma\right]^{1 / 2} \leq\left[\max _{z_{0} \geq s \geq z_{1}} \xi(s)\right]\left[2 \int_{z_{0}}^{Z(v, \tau)} q(\sigma) d \sigma\right]^{1 / 2}
\end{gathered}
$$

Isso completa a demonstração do lema.

Lema 4.3. Sejam $\xi, \eta:\left[x_{0}, X\right) \rightarrow \mathbb{R}$ funções não negativas, diferenciáveis sobre $\left[x_{0}, X\right)$. Suponha que $\xi$ e $\eta$ são absolutamente contínuas e exitam funções $a, b:\left[x_{0}, X\right) \rightarrow \mathbb{R}$ não negativas e integráveis em cada intervalo $\left[x_{0}, x\right] \operatorname{com} x \leq X$; colocando $\xi_{0}=\xi\left(x_{0}\right), \eta_{0}=\eta\left(x_{0}\right)$ e se

$$
\xi^{\prime}(x) \leq a(x) \eta(x) \eta^{\prime}(x) \leq b(x) \xi(x)
$$

então para $x_{0} \leq x<X$ temos

$$
\begin{gathered}
\xi(x) \leq\left[\xi_{0}+\eta_{0} \int_{x_{0}}^{x} a(s) d s\right] \exp \left[\int_{x_{0}}^{x} a(s) \int_{0}^{s} b(\sigma) d \sigma d s\right] \\
\eta(x) \leq \eta_{0}+\left[\xi_{0}+\eta_{0} \int_{x_{0}}^{x} a(s) d s\right]\left[\int_{x_{0}}^{x} b(s) d s\right] \exp \left[\int_{x_{0}}^{x} a(s) \int_{0}^{s} b(\sigma) d \sigma d s\right]
\end{gathered}
$$

Demonstração. Vamos introduzir a notação

$$
g(x)=\max _{x_{0} \leq s \leq x} \xi(s)
$$

Então

$$
\eta(x) \leq \eta_{0}+\int_{x_{0}}^{x} b(s) \xi(s) d s \leq \eta_{0}+g(x) \int_{x_{0}}^{x} b(s) d s
$$

Dessa maneira segue que 


$$
\eta^{\prime}(x) \leq a(x)\left[\eta_{0}+g(x) \int_{x_{0}}^{x} b(s) d s\right]=a(x) \eta_{0}+a(x) g(x) \int_{x_{0}}^{x} b(s) d s
$$

e assim

$$
\begin{aligned}
g(x)=\max _{x_{0} \leq y \leq x} \xi(y) & =\max _{x_{0} \leq y \leq x}\left\{\xi_{0}+\int_{x_{0}}^{y} \xi^{\prime}(s) d s\right\} \\
& \leq \max _{x_{0} \leq y \leq x}\left\{\xi_{0}+\int_{x_{0}}^{y} a(s) \eta_{0} d s+a(s) g(s) \int_{x_{0}}^{s} b(\sigma) d \sigma\right\} \\
& \leq \xi_{0}+\eta_{0} \int_{x_{0}}^{x} a(s) d s+\int_{x_{0}}^{x} a(s) g(s) \int_{x_{0}}^{s} b(\sigma) d \sigma d s
\end{aligned}
$$

Assim temos que

$$
g(x) \leq \xi_{0}+\eta_{0} \int_{x_{0}}^{x} a(s) d s+\int_{x_{0}}^{x} a(s) \int_{x_{0}}^{s} b(\sigma) d \sigma \cdot g(s) d s
$$

Por um lema de Bellman-Gronwall segue que

$$
g(x) \leq\left[\xi_{0}+\eta_{0} \int_{x_{0}}^{x} a(s) d s\right] \exp \int_{x_{0}}^{x} a(s) \int_{x_{0}}^{s} b(\sigma) d \sigma
$$

Isso prova (80). Substituindo (83) em (82) obtemos (81). Isso completa a prova do lema.

Lema 4.4. As derivadas parciais $\frac{\partial h^{ \pm}}{\partial v}(y, v, \tau) e \frac{\partial h^{ \pm}}{\partial \tau}(y, v, \tau)$ de $h^{ \pm}(y, v, \tau)$ são contínuas com respeito a $(y, v, \tau)$ na região $\left\{v>0, y \neq Z^{ \pm}(v, \tau)\right\}$, e sobre a superfície $y=Z^{ \pm}(v, \tau)$ tais derivadas tem limites de ambos os lados (para $v>0$ ) que são contínuas (e, em geral, distintas). Lembremos da equação (30) que é dada abaixo.

$$
h^{ \pm}(y, v, \tau)=\left\{\begin{array}{cc}
\frac{V(y, v, \tau)^{2}}{2}+\int_{0}^{y} Q_{0}(z) d z, & 0 \leq y \leq Z^{ \pm}(v, \tau) \\
\int_{0}^{Z^{ \pm}(v, \tau)} Q_{0}(z) d z & Z^{ \pm}(v, \tau)<y
\end{array}\right.
$$

Demonstração. Mais uma vez omitiremos os símbolos \pm das funções, por motivos já explicitados.

Para $y>Z(v, \tau)$ segue de $(30)$ que

$$
h(y, v, \tau) \equiv h(v, \tau) .
$$

Usando a notação $\delta$ para substituir os símbolos de derivadas parciais com respeito a $v$ e também com respeito a $\tau$ nós temos que, para $y>Z(v, \tau)$ 


$$
\delta h(y, v, \tau)=\delta h(v, \tau)=Q_{0}(Z(v, \tau)) \delta Z .
$$

Essas derivadas são contínuas, pelo corolário 4.1. Observe que os valores de $(v, \tau)$ são tomados em $R_{0}$, onde faz sentido diferenciar a função $Z$. Denotaremos

$$
\delta h_{\text {int }}=Q_{0}(Z) \delta Z
$$

Por outro lado, diferenciando as identidades

$$
z(t(y, v, \tau), v, \tau) \equiv y, \quad \dot{z}(t(y, v, \tau), v, \tau)=V(y, v, \tau)
$$

que definem $t(y, v, \tau)$ e $V(y, v, \tau)$, concluímos que para $0 \leq y \leq Z(v, \tau)$

$$
\delta V=\frac{\partial z}{\partial t} \delta t+\delta \dot{z}, \quad \dot{z}(t, v, \tau) \delta t+\delta z=0
$$

Segue disso então que

$$
\begin{gathered}
\delta t=-\frac{\delta z}{V} \\
\delta V=-\frac{Q(z(t(y, v, \tau), t(y, v, \tau))) \delta z}{V(y, v, \tau)}=\delta \dot{z}+\frac{Q}{V} \delta z .
\end{gathered}
$$

Dessa forma para $0 \leq y \leq Z(v, \tau)$

$$
\delta h(y, v, \tau)=V \delta V=Q(y, t(y, v, \tau)) \delta Z+V(y, v, \tau) \delta z .
$$

Conseqüentemente, essas derivadas são contínuas para $0 \leq y<Z(v, \tau)$ e a partir do lado externo da superfície $y=Z(v, \tau)$ encontramos os limites

$$
\delta h_{e x t}=Q(y, T(v, \tau)) \delta Z, y=Z(v, \tau)
$$

Neste caso

$$
V(T(v, \tau), v, \tau)=0 .
$$

Façamos agora a seguinte estimativa 


$$
\Delta=\delta h_{e x t}-\delta h_{i n t}=Q(y, T(v, \tau)) \delta Z-Q_{0}(Z) \delta Z=\frac{Q_{0}(Z)-Q(Z, T)}{Q(Z, T)} \delta h_{e x t}
$$

Assim temos que

$$
|\Delta| \leq \frac{2 \pi}{q(Z)} \psi(Z) \delta h_{e x t} \leq 2 \pi \xi(Z) q(Z) \delta h_{\text {ext }}
$$

Temos assim provado o lema.

De tudo que temos provado nesta seção, estamos prontos para provar o seguinte teorema:

Teorema 4.1. As funções $h^{ \pm}$são de classe $C^{1}(\Phi \backslash\{O\})$ e as curvas de nível destas funções são difeomorfas a círculos.

Demonstração. Como na prova da continuidade de $h$ dadas na proposição 2.3, para obter informações a respeito de $h(v, \tau)$ é natural passarmos o limite

$$
h(v, \tau)=\lim _{y \rightarrow+\infty} h(y, v, \tau)
$$

A situação é complicada pelo fato de que as derivadas de $h(y, v, \tau)$ são descontínuas sobre a superfície $y=Z(v, \tau)$.

Na região

$$
0 \leq y \leq Z(v, \tau)
$$

as funções $h(y, v, \tau)$ e $t(y, v, \tau)$ satisfazem o sistema de equações diferenciais

$$
\left\{\begin{array}{l}
\frac{d h}{d y}=Q_{0}(y)-Q(y, t(y, v, \tau)) \\
\frac{d t}{d y}=\quad \frac{1}{V}
\end{array}\right.
$$

O qual vem das equações (32) e (26).

De (32) ainda temos que

$$
V=\left[2\left(h-\int_{0}^{y} Q_{0}(\xi) d \xi\right)\right]^{1 / 2}
$$

Assim $\delta h$ e $\delta t$ podem ser encontrados a partir do sistema de equações variacionais:

$$
(\delta h)^{\prime}=-Q_{t}^{\prime}(y, t) \delta t, \quad(\delta t)^{\prime}=-\frac{\frac{1}{2}\left[2\left(h-\int_{0}^{y} Q_{0}(\xi) d \xi\right)\right]^{-1 / 2} 2 \delta h}{V^{2}}=-\frac{\delta h}{V^{3}}
$$


Onde a derivada reperesentada por uma linha, é a derivada com relação a $y$.

Por meio de $4^{\mathrm{o}}$ obtemos

$$
|\delta h|^{\prime} \leq\left|(\delta h)^{\prime}\right| \leq \psi(y)|\delta t| ; \quad|\delta t|^{\prime} \leq\left|(\delta t)^{\prime}\right| \leq \frac{1}{V^{3}(y)}|\delta h|
$$

Usando o lema $4.3 \operatorname{com} \xi=|\delta h|, a(y)=\psi(y), \eta=\delta t$ e $b(y)=\frac{1}{V^{3}(y)}$ e daí para $0 \leq y_{0} \leq$ $y \leq Z$ temos

$$
\begin{gathered}
|\delta h(y)| \leq\left[\left|\delta h\left(y_{0}\right)\right|+\left|\delta t_{0}\right| \int_{y_{0}}^{y} \psi(s) d s\right] \exp \int_{y_{0}}^{y} \psi(s) \int_{y_{0}}^{s} \frac{d \sigma d s}{V^{3}(\sigma)} \\
|\delta t(y)| \leq\left|\delta t_{0}\right|+\int_{y_{o}}^{y} \frac{d s}{V^{3}(s)}\left[\left|\delta h\left(y_{0}\right)\right|+\delta t\left(y_{0}\right) \int_{y_{0}}^{y} \psi(s) d s\right] \exp \int_{y_{0}}^{y} \psi(s) \int_{y_{0}}^{s} \frac{d \sigma d s}{V^{3}(\sigma)}
\end{gathered}
$$

Na região $R_{0}=\{(v, \tau) ; \quad 0<h(v, \tau)<\Im\}$ as condições necessárias sobre $h(v, \tau)$ são estabelecidas pelo corolário 4.1. Estamos assumindo que $\Im$ é finito. Em vista da condição $6^{\circ}$, $Q(z, t)$ é decrescente em $z$ para $z \leq z^{*}$. O mesmo é verdadeiro para $Q_{0}(z)$. Desde que $Q_{0}(z)$ é integrável e decrescente então

$$
\lim _{z \rightarrow+\infty} Q_{0}(z)=0
$$

Pela $3^{\text {oo }}$ condição $0<q(z) \leq Q_{0}(z)$ e assim

$$
\int_{0}^{+\infty} q(z) d z<+\infty \quad \lim _{z \rightarrow+\infty} q(z)=0
$$

Para $y_{0}$ seja $z^{*}$ definido nas condições $5^{\circ}$ e $6^{\circ}$. Como $\Im$, por $5^{\circ}$, para $z \geq z^{*}$ temos que $\xi(z) \leq C$, para alguma constante $C>0$ suficientemente grande. Pelo lema 4.2 e (92) temos

$$
\int_{z^{*}}^{y} \psi(s) \int_{z^{*}}^{s} \frac{d \sigma d s}{V^{3}(\sigma)} \leq C\left[2 \int_{Z^{*}}^{Z} q(\sigma) d \sigma\right]^{1 / 2} \leq C\left[2 \int_{0}^{+\infty} q(\sigma) d \sigma\right]<+\infty
$$

para $z^{*} \leq y \leq Z(v, \tau)$. Seja $R>0$ arbitrário e

$$
K_{R}=\left\{(v, \tau) ; \quad z^{*}+1 \leq Z(v, \tau), v \leq R\right\}
$$

$K_{R}$ é um conjunto compacto.

Fora da curva $z^{*}=X(v, \tau)$, e conseqüentemente sobre o compacto $K_{R}$, as funções $\delta h\left(z^{*}, v, \tau\right)$ e $\delta t\left(x^{*}, v, \tau\right)$ são contínuas nas variáveis $(v, \tau)$, pelo lema 4.4 . 
Dessa forma

$$
\begin{gathered}
M_{1}=\sup _{K_{R}}\left|\delta t\left(z^{*}, v, \tau\right)\right|<+\infty \\
M_{2}=\sup _{K_{R}}\left[\left|\delta h\left(z^{*}, v, \tau\right)\right|+\left|\delta t\left(z^{*}, v, \tau\right)\right| \int_{0}^{+\infty} \psi(s) d s\right] \exp C\left[2 \int_{0}^{+\infty} q(\sigma) d \sigma\right]^{1 / 2}<+\infty
\end{gathered}
$$

Seja $M=\max \left\{M_{1}, 2 C M_{2}\right\}$. Em (90) e (91) coloquemos $y_{0}=x^{*}$ e aplicando (93) segue que

$$
\begin{gathered}
|\delta h(y, v, \tau)| \leq M_{2} \\
|\delta t(y, v, \tau)| \leq M_{1}+M_{2} \int_{z^{*}}^{y} \frac{d \sigma}{V^{3}(\sigma)}
\end{gathered}
$$

e por (89) temos

$$
\left|(\delta h)^{\prime}\right| \leq M_{1} \psi(y)+M_{2} \psi(y) \int_{z^{*}}^{y} \frac{d \sigma}{V^{3}(\sigma)}
$$

O lado direito de (96) é integrável, logo $\left|\delta h^{\prime}\right|$ é integrável e isso implica que $\delta h^{\prime}$ é integrável sobre o intervalo $\left[z^{*}, Z\right)$. Então, se $Z(v, \tau)=+\infty$ existe o limite

$$
\delta h(+\infty)=\lim _{y \rightarrow+\infty} \delta h(y)
$$

e este limite é finito.

Se $Z(v, \tau)<+\infty$, então $\delta h(y)$ tem limite $\delta h_{e x t}=\lim _{y \rightarrow Z(v, \tau)_{-}} \delta h(y)$ onde $y \rightarrow Z(v, \tau)_{-}$denota o limite lateral à esquerda. O valor $\delta h_{e x t}$ coincide com o valor dada em (85) do lema 4.4. Agora aplicamos (94) e obtemos

$$
\delta h_{\text {ext }} \leq M_{2}
$$

Quando percorremos a superfície $y=Z(v, \tau)$ a função $\delta h(y, v, \tau)$ possui um salto de tamanho estimado em $\Delta$ dado em (86). Finalmente para $y>Z(v, \tau)$

$$
\delta h(y, v, \tau)=\delta\left(\int_{0}^{Z(v, \tau)} Q_{0}(z) d z\right)=Q_{0}(Z) \delta Z
$$

Conseqüentemente no caso $Z(v, \tau) \leq+\infty$ o limite $(97)$ existe. 
Temos que $\delta h_{i n t}=\delta h_{e x t}-\Delta$. Então segue que

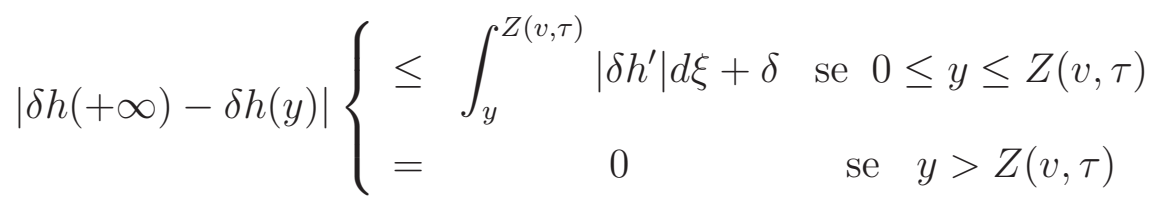

De fato,

$$
\begin{gathered}
|\delta h(+\infty)-\delta h(y)| \leq|\delta h(+\infty)-\delta h(Z)|+|\delta h(Z)-\delta h(y)| \leq|\delta h(+\infty)-\delta h(Z)|+\left|\int_{y}^{Z} \delta h^{\prime} d \xi\right| \leq \\
\quad \leq \int_{y}^{Z}\left|\delta h^{\prime}\right| d \xi+|\delta h(+\infty)-\delta h(Z)| \leq \int_{y}^{Z}\left|\delta h^{\prime}\right| d \xi+\left|\delta h_{i n t}-\delta h_{e x t}\right|=\int_{y}^{Z}\left|\delta h^{\prime}\right| d \xi+\Delta
\end{gathered}
$$

pois neste caso $Z=Z(v, \tau)<+\infty$.

Para estimar $\left|\delta h^{\prime}\right|$ podemos usar (96) e para estimar $\Delta$ nós usamos (86). Para $y \leq z^{*}+1$ e $(v, \tau) \in K_{R}$ temos

$$
\begin{aligned}
& \mid \delta h(+\infty, v, \tau)- \delta h(y, v, \tau) \mid \leq M_{1} \int_{y}^{Z} \psi(s) d s+M_{2} \int_{y}^{Z} \psi(s) \int_{z^{*}}^{s} \frac{d \sigma d s}{V^{3}(\sigma)}+2 \pi C q(y) M_{2} \\
& \leq M\left[\int_{y}^{+\infty} \psi(s) d s+q(y)\right]+M_{2} \int_{y}^{Z} \psi(s) \int_{z^{*}}^{s} \frac{d \sigma d s}{V^{3}(\sigma)}
\end{aligned}
$$

Novamente, aplicando o lema 4.2 para a segunda integral, nós obtemos:

$$
|\delta h(+\infty, v, \tau)-\delta h(y, v, \tau)| \leq M\left[\int_{y}^{+\infty} \psi(s) d s+q(y)+\left(\int_{y}^{+\infty} q(\sigma) d \sigma\right)^{1 / 2}\right]
$$

Como $q$ e $\psi$ são funções integráveis e $\lim _{y \rightarrow+\infty} q(y)$ então a desigualdade acima mostra que $\delta h(y, v, \tau) \rightarrow \delta h(+\infty, v, \tau)=\delta h$ quando $y \rightarrow+\infty$, e esta convergência não depende de $(v, \tau)$, logo ,a convergência é uniforme nestas variáveis, sobre o compacto $K_{R}$. O salto $\Delta \rightarrow 0$ uniformemente quando $y \rightarrow+\infty$, assim

$$
\delta h_{e x t}(+\infty, v, \tau) \equiv \delta h_{i n t}(+\infty, v, \tau)
$$

Isso implica que $\delta h$ é contínua sobre $y=Z(v, \tau)$

Assim $\delta h$ é contínua sobre $K_{R}$ como tomamos $R>0$ arbitrário concluímos que $\delta h \in$ $C^{1}(\Phi \backslash\{O\})$. Como desejávamos. 
Resta agora provar que $\nabla h(v, \tau) \neq 0$ para todo $(v, \tau) \in \Phi \backslash\{O\}$ e usaremos este fato para provar que todas as curvas de nível definidas por

$$
h(v, \tau)=C>0
$$

são difeomorfas a um círculo.

Observemos que, pelo corolário 4.1 o gradiente de $h$ é não nulo na região $R_{0}$. Para provar o resultado desejado vamos supor $\Im<+\infty$ e $Z^{+}(v, \tau)=+\infty$. Escolhamos um $y_{0}$ suficientemente grande tal que

$$
y_{0} \geq z^{*}
$$

As derivadas parciais $\frac{\partial h}{\partial v}, \frac{\partial h}{\partial \tau}, \frac{\partial t}{\partial v}, \frac{\partial t}{\partial \tau}$ são soluções do sistema variacional (88) e dessa forma o determinate

$$
\frac{\partial(h, t)}{\partial(v, \tau)} \neq 0
$$

para $0 \leq y<Z=+\infty$.

Conseqüentemente existem $\alpha$ e $\beta$ tais que

$$
\left(\begin{array}{c}
\delta h \\
\delta t
\end{array}\right)=\alpha\left(\begin{array}{c}
\frac{\partial h}{\partial v} \\
\frac{\partial t}{\partial v}
\end{array}\right)+\beta\left(\begin{array}{c}
\frac{\partial h}{\partial \tau} \\
\frac{\partial t}{\partial \tau}
\end{array}\right)
$$

Sendo

$$
\left(\begin{array}{l}
\delta h \\
\delta t
\end{array}\right)=\left(\begin{array}{l}
1 \\
0
\end{array}\right)
$$

Para $y=y_{0}$.

O par $\left(\begin{array}{c}\delta h \\ \delta t\end{array}\right)$ é solução de (88), dessa forma, as estimativas (90) e (91) se verficam. Desde que $\delta h(0)=1, \delta t(0)=0$, para $y \geq y_{0}$ de (91) e (89) segue que

$$
|\delta t| \leq \int_{y_{0}}^{y} \frac{d s}{v^{3}(s)} \cdot \exp \int_{y_{0}}^{y} \psi(s) \int_{y_{0}}^{s} \frac{d \sigma d s}{V^{3}(\sigma)}
$$




$$
\left|\delta h^{\prime}\right| \leq \psi(y) \int_{y_{0}}^{y} \frac{d s}{v^{3}(s)} \cdot \exp \int_{y_{0}}^{y} \psi(s) \int_{y_{0}}^{s} \frac{d \sigma d s}{V^{3}(\sigma)}
$$

Por aplicação do teorema fundamental do cálculo e equação (89) temos

$$
\left|\delta h(+\infty)-\delta h\left(y_{0}\right)\right| \leq \int_{y_{0}}^{+\infty}\left(\psi(y) \int_{y_{0}}^{y} \frac{d s}{v^{3}(s)} \cdot \exp \int_{y_{0}}^{+\infty} \psi(s) \int_{y_{0}}^{s} \frac{d \sigma d s}{V^{3}(\sigma)}\right) d y
$$

Lembremos que $\psi$ é integrável em $[0,+\infty)$ e pela parte 2 do lema 4.2 segue que

$$
\lim _{y_{0} \rightarrow+\infty} \psi(y) \int_{y_{0}}^{y} \frac{d s}{V^{3}(s)} \cdot \exp \int_{y_{0}}^{+\infty} \psi(s) \int_{y_{0}}^{s} \frac{d \sigma d s}{V^{3}(\sigma)}=\psi(y) \int_{y_{0}}^{y} \frac{d s}{V^{3}(s)}
$$

Assim tomando $\varepsilon$ suficientemente pequeno, encontramos $y_{0} \geq z^{*}$ tal que

$$
\left|\delta h(+\infty)-\delta h\left(y_{0}\right)\right| \leq(1+\varepsilon)\left(\psi(y) \int_{y_{0}}^{+\infty} \frac{d s d y}{V^{3}(s)}\right)<\frac{1}{4}
$$

desde que $y_{0}$ seja suficientemente.

Assim pela desigualdade triangular obtemos

$$
\left|\delta h\left(y_{0}\right)\right|-|\delta h(+\infty)|<\frac{1}{4} \Rightarrow|\delta h(+\infty)|>\left|\delta h\left(y_{0}\right)\right|-\frac{1}{4}=\frac{3}{4} \Rightarrow|\delta h(+\infty)|>1-\frac{1}{4}=\frac{3}{4}
$$

Com isso concluímos que $\nabla h(v, \tau) \neq 0$ qualquer que seja $(v, \tau) \in \Phi \backslash\{O\}$.

Passemos agora à demonstração de que as curvas de nível definidas pela equação $h(v, \tau)=$ $C>0$ são difeomorfas a círculos. Para o caso $0<C<\Im$ sabemos que o teorema é verdadeira. Então para que o teorema se verifique em todos os casos, basta mostrar que todas as curvas de nível são difeomorfas.

Sejam $0<C_{1}<\Im \leq C_{2}$ números reais arbitrários fixados. Por continuidade de $h$, podemos encontrar números $0<r<R$ tais que

$$
h(v, \tau)<C_{1}, \quad 0 \leq v<r
$$

e

$$
h(v, \tau)>C_{2} \text { fora do anel } r \leq v \leq R
$$

Com auxílio de uma função do tipo bump, vamos aproximar as funções 


$$
A=\frac{h_{v}^{\prime}}{|\nabla h|}, \quad B=\frac{h_{\tau}^{\prime}}{|\nabla h|}
$$

por funções $\widetilde{A}$ e $\widetilde{B}$ tais que no anel $r \leq v \leq R$, as funções $\widetilde{A}$ e $\widetilde{B}$ coincidam com $A$ e $B$ respectivamente, e para um $\varepsilon>0$ pequeno $\widetilde{A}$ e $\widetilde{B}$ se anulem fora de $r-\varepsilon<v<R+\varepsilon$. Vamos considerar um sistema auxiliar.

$$
\left\{\begin{array}{l}
\frac{d v}{d \alpha}=-\widetilde{B} \\
\frac{d \tau}{d \alpha}=\widetilde{A}
\end{array}\right.
$$

Seja $(v(s, \alpha), \tau(s, \alpha))$, uma família de soluções tais que para algum $\alpha_{0}$ tenhamos

$$
h\left(\left(v\left(0, \alpha_{0}\right), \tau\left(0, \alpha_{0}\right)\right)\right)=C_{1}
$$

então essas solução satisfaz

$$
h(v(0, \alpha), \tau(0, \alpha))=C_{1}
$$

De fato

$$
\frac{d}{d \alpha} h(v(0, \alpha), \tau(0, \alpha))=-\widetilde{A} \widetilde{B}+\widetilde{A} \widetilde{B}=0
$$

logo $h(v(0, \alpha), \tau(0, \alpha))$ é constante igual a $C_{1}$. Observemos, do sistema (100) que as solução $v(0, \alpha), \tau(0, \alpha)$ está definido para todo $\alpha$. Mas isso a trajetrória é uma parametrização para a curva de nível $h(v, \tau)=C_{1}$. Sabemos que o traço da curva $\alpha \mapsto v(0, \alpha), \tau(0, \alpha)$ está contido no traço da curva de nível $h(v, \tau)=C_{1}$, se a inclusão fosse estrita, geraríamos um ponto singular nas extremidades, isso significa que o campo $(-\widetilde{B}, \widetilde{A})$ teria um zero no anel $r \leq v \leq R$, mas neste anel temos $(-\widetilde{B}, \widetilde{A})=(-B, A) \neq 0$, pois $\nabla h \neq 0$ no anel.

Consideremos agora o sistema

$$
\left\{\begin{array}{l}
\frac{d v}{d s}=\widetilde{A} \\
\frac{d \tau}{d s}=\widetilde{B}
\end{array}\right.
$$


Assim, ajustando convenientemente as condições iniciais, obtemos uma família de soluções no anel $r \leq v \leq R$, tais que para cada $s$ fixado $(v(s, \alpha), \tau(s, \alpha))$ são parametrizações das curvas de nível $h(v, \tau)=C$, com $C_{1}<C<C_{2}$. Notemos que podemos resolver os sistema (100) e (101) supondo que $(v(s, \alpha), \tau(s, \alpha))=(v(s)+v(\alpha), \tau(s)+\tau(\alpha))$.

Como $\widetilde{A}$ e $\widetilde{B}$ coincidem com $A$ e $B$ em $r \leq v \leq R$ notemos que

$$
\frac{\partial h}{\partial s}(v(s, \alpha), \tau(s, \alpha))=\frac{\partial h}{\partial v} \cdot A+\frac{\partial h}{\partial \tau} B=A^{2}+B^{2}=1
$$

Sabemos que $h\left(v(0, \alpha), \tau(0, \alpha)=C_{1}\right.$, assim pelo teorema de existência e unicidade, para cada $\alpha$, existe um único $s(\alpha)$ tal que $h\left(v(s(\alpha), \alpha), \tau(s(\alpha), \alpha)=C_{2}\right.$. Pela equação (102) podemos aplicar o teorema da função implícita e concluir que $\alpha \mapsto s(\alpha)$ é um difeomorfismo local, e da equação

$$
h\left(v(s(\alpha), \alpha), \tau(s(\alpha), \alpha)=C_{2} .\right.
$$

concluímos que

$$
\frac{d s}{d \alpha}=-\frac{h_{v}^{\prime} v_{\alpha}^{\prime}+h_{\tau}^{\prime} v_{\alpha}^{\prime}}{A h_{v}^{\prime}+B h_{v}^{\prime}}=0
$$

Esse fato pode ser interpretado como paralelismo entre as curvas de nível.

A aplicação $(v(0, \alpha), \tau(0, \alpha)) \mapsto(v(s(\alpha), \alpha), \tau(s(\alpha), \alpha))$ é injetora. De fato

Se $\left.\left.v\left(s\left(\alpha_{1}\right), \alpha_{1}\right), \tau\left(s\left(\alpha_{1}\right), \alpha_{1}\right)\right)=v\left(s\left(\alpha_{2}\right), \alpha_{2}\right), \tau\left(s\left(\alpha_{2}\right), \alpha_{2}\right)\right)$, como $\left.v\left(s\left(\alpha_{1}\right), \alpha_{1}\right), \tau\left(s\left(\alpha_{1}\right), \alpha_{1}\right)\right)$ é obtido das soluções das equações diferenciais (100) e (101), segue do teorema de existência e unicidada para equações diferenciais ordinárias que

$$
\left(s\left(\alpha_{1}\right), \alpha_{1}\right)=\left(s\left(\alpha_{2}\right), \alpha_{2}\right) \Rightarrow \alpha_{1}=\alpha_{2} .
$$

Assim, da arbitrariedade da escolha de $C_{1}$ e $C_{2}$, concluímos que a aplicação

$$
(v(0, \alpha), \tau(0, \alpha)) \mapsto(v(s(\alpha), \alpha), \tau(s(\alpha), \alpha))
$$

é um difeomorfismo entre as curvas de nível $h(v, \tau)=C_{1}$ e $h(v, \tau)=C_{2}$. Isso encerra a demonstração. 


\subsection{Propriedades Diferenciais da Aplicação $S$.}

Procedemos agora com a investigação das propriedades diferenciais da função sucessor $S$. Desde que $S$ está definida em termos da solução $z(t, v, \tau)$ primeiro estabeleceremos algumas propriedades das derivadas de $z(t, v, \tau)$ com respeito a $v$ e $\tau$.

Se $v$ e $\tau$ dependem diferenciavelmente de um parâmetro $s$, então

$$
\partial z=\frac{\partial}{\partial s}(z(t, v(s), \tau(s)))
$$

é solução da equação variacional.

$$
\delta \ddot{z}=-Q_{z}^{\prime}(z(t, v, \tau), t) \delta z
$$

De fato, temos que

$$
\begin{gathered}
\partial z=\frac{\partial z}{\partial v} \frac{\partial v}{\partial s}+\frac{\partial z}{\partial \tau} \frac{\partial \tau}{\partial s} \\
\ddot{z}=-Q(z, t)
\end{gathered}
$$

segue que

$$
\frac{\partial \ddot{z}}{\partial s}=-Q_{z}^{\prime}(z(t, v, \tau), t)\left(\frac{\partial z}{\partial v} \frac{\partial v}{\partial s}+\frac{\partial z}{\partial \tau} \frac{\partial \tau}{\partial s}\right)=-Q_{z}^{\prime}(z(t, v, \tau), t) \partial z .
$$

A solução $\partial z(t, v, \tau)$ depende continuamente das condições iniciais acima sobre $(v, \tau) \in \Phi$. A aplicação $S$ está definida sobre $R_{0}^{+}$, queremos estudar o comportamento de $S$ quando $(v, \tau)$ se aproxima de $\Pi_{0}^{+}$, no caso em que $\Im<+\infty$.

Seja

$$
p(t, v, \tau)=\left\{\begin{array}{ccc}
-Q_{z}^{\prime}(z(t, v, \tau), t) & \text { se } & 0 \leq v<+\infty \\
0 & \text { se } & v=+\infty
\end{array}\right.
$$

Lema 4.5. Uma solução $\omega(t, v, \tau)$ da equação

$$
\ddot{\omega}=p(t, v, \tau) \omega
$$

depende continuamente de $(v, \tau)$ e das condições iniciais

$$
\omega_{0}=\omega(\tau, v, \tau) ; \dot{\omega}_{0}=\dot{\omega}(\tau, v, \tau)
$$


A demonstração deste lema é conseqüência da teoria das equações variacionais.

Lema 4.6. Se na equação

$$
\ddot{\omega}=p(t) \omega
$$

$p(t)$ é não negativa para todo $t \geq t_{0}$ então as seguintes afirmações são válidas

1) Para cada solução de (106) o limite $\lim _{t \rightarrow+\infty} \omega(t)=\omega(\infty)$ (finito ou infinito) existe.

2) Existe uma solução $\omega_{1}(t)$ não trivial, não negativa para $t$ suficientemente grande e $\omega_{1}(\infty)=$ 0 , aqui $\omega_{1}(t)>0$ e $\dot{\omega}_{1}(t) \leq 0$ para $t \geq t_{0}, t_{0}$ suficientemente grande.

3) Para uma solução arbitrária $\omega_{2}$ de (106) temos

$$
\operatorname{sgn}\left(\dot{\omega}_{2}(\infty)\right)=\left|\begin{array}{cc}
\omega_{1} & \omega_{2} \\
\dot{\omega}_{1} & \dot{\omega}_{2}
\end{array}\right|
$$

Demonstração. Seja $\left\{\omega_{3}(t), \omega(t)\right\}$ um sistema fundamental de soluções sobre $t=t_{0}$ determinado pelas condições iniciais

$$
\left(\begin{array}{cc}
\omega_{3}\left(t_{0}\right) & \omega_{4}\left(t_{0}\right) \\
\dot{\omega}_{3}\left(t_{0}\right) & \dot{\omega}_{4}\left(t_{0}\right)
\end{array}\right)=\left(\begin{array}{cc}
1 & 0 \\
0 & 1
\end{array}\right)
$$

Para $t \geq t_{0}$ cada solução de (106) tem no máximo um zero, a partir deste ponto e o seu sinal fica constante.

De fato, como vamos supor que $v$ é finito, $p(t)$ é positiva, então, ambos $\omega$ e omëga tem o mesmo sinal para $t \geq t_{0}$. Sabemos, pelo teorema de existência e unicidade de equações diferenciais que não pode ocorrer uma solução não trivial de (106) que tangencie o eixo $t$, isto é, $\omega\left(t_{0}\right)=0$ e $\dot{\omega}\left(t_{0}\right)=0$. Assim, se $\omega$ tem mais de um zero em $t_{1} \leq t_{2} \operatorname{com} t_{1} \geq t_{0}$, temos que: se em $\left(t_{1}, t_{2}\right)$ a função $\omega$ for positiva então este é concavo para baixo, isto é $\ddot{\omega} \leq 0$ em $\left(t_{1}, t_{2}\right)$, o que não pode ocorrer. Se em $\left(t_{1}, t_{2}\right)$ a função $\omega$ for negativa, obtemos uma contradição análoga.

Suponha que $\omega(t)>0$, para todo $t \geq t_{0}$. Daí temos que

$$
\ddot{\omega}=p \omega \leq 0
$$


isso implica que $\dot{\omega}$ é não decrescente assim, admitindo o símbolo $+\infty$ como valor limite, exite o limite

$$
\lim _{t \rightarrow+\infty} \dot{\omega}(t)=\dot{\omega}(+\infty)
$$

Temos então provado o item 1) do lema.

Em particular, o zero de $\omega_{4}(t)$ para $t \geq t_{0}$ ocorre em $t=t_{0}$ já que $\dot{\omega}_{4}\left(t_{0}\right)=1>0$. Daí segue que

$$
\lim _{t \rightarrow+\infty} \omega_{4}(t)=+\infty .
$$

Temos, da independência do determinante Wronskiano, que

$$
\begin{aligned}
& \frac{d}{d t}\left(\frac{\omega_{3}}{\omega_{4}}\right)=\frac{\omega_{3} \dot{\omega}_{4}-\omega_{4}-\dot{\omega}_{3}}{\omega_{4}^{2}}=-\frac{\left|\begin{array}{cc}
\omega_{3} & \omega_{4} \\
\dot{\omega}_{3} & \dot{\omega}_{4}
\end{array}\right|}{\omega_{4}^{2}}=-\frac{1}{\omega_{4}^{2}}<0 \\
& \frac{d}{d t}\left(\frac{\dot{\omega}_{3}}{\dot{\omega}_{4}}\right)=\frac{\omega_{3} \dot{\omega}_{4}-\omega_{4} \dot{\omega}_{3}}{\omega_{4}^{2}}=\frac{p}{\omega_{4}^{2}}\left|\begin{array}{cc}
\omega_{3} & \omega_{4} \\
\dot{\omega}_{3} & \dot{\omega}_{4}
\end{array}\right|=\frac{p}{\omega_{4}^{2}}>0 \\
& \frac{\omega_{3}}{\omega_{4}}-\frac{\dot{\omega}_{3}}{\dot{\omega}_{4}}=\frac{\omega_{3} \dot{\omega}_{4}-\omega_{4} \dot{\omega}_{3}}{\omega_{4} \dot{\omega}_{4}}=\quad \frac{\left|\begin{array}{ll}
\omega_{3} & \omega_{4} \\
\dot{\omega}_{3} & \dot{\omega}_{4}
\end{array}\right|}{\omega_{4} \dot{\omega}_{4}}=\frac{1}{\omega_{4} \dot{\omega}_{4}} \downarrow 0
\end{aligned}
$$

Daí temos que existe um limite comum

$$
\alpha=\lim _{t \rightarrow+\infty} \frac{\omega_{3}}{\omega_{4}}=\frac{\dot{\omega}_{3}}{\dot{\omega}_{4}}
$$

Observe que $\alpha$ é finito pois $\frac{\omega_{3}\left(t_{0}+\varepsilon\right)}{\omega_{4}\left(t_{0}+\varepsilon\right)}>0$ para $\varepsilon>0$ pequeno.

Ainda temos que

$$
\frac{\omega_{3}}{\omega_{4}} \geq \alpha \geq \frac{\dot{\omega}_{3}}{\dot{\omega}_{4}}
$$

Assim, a solução

$$
\omega_{1}=\omega_{3}(t)-\alpha \omega_{4}(t)>0
$$


e sua derivada

$$
\dot{\omega}_{1}=\dot{\omega}_{3}-\alpha \omega_{4} \leq 0
$$

Ambas as desiguldades ocorrendo para $t \geq t_{0}$. A partir da primeira desigualdade $\omega_{1}(+\infty)$ não pode ser negativo e a segunda não pode ser positiva. Daí $\omega_{1}(\dot{+} \infty)=0$ Temos provado o segundo item deste lema.

Para uma solução $\omega_{2}(t)$ de (106) nós temos

$$
\begin{gathered}
\omega_{2}(t)=\omega_{2}\left(t_{0}\right) \omega_{3}(t)+\dot{\omega}_{2} \omega_{4}(t)=\omega_{2}\left(t_{0}\right)\left[\omega_{3}(t)-\alpha \omega_{4}(t)\right]+\left[\omega_{2}\left(t_{0}\right)+\alpha \omega_{2}\left(t_{0}\right)\right] \omega_{4}(t)= \\
=\omega_{2}\left(t_{0}\right) \omega_{1}(t)+\left|\begin{array}{cc}
1 & \omega_{2}\left(t_{0}\right) \\
-\alpha & \dot{\omega}_{2}\left(t_{0}\right)
\end{array}\right| \omega_{4}=
\end{gathered}
$$

Pela independência de $t$ do determinante Wrosnkiano a igualdade acima se reduz a

$$
=\omega_{2}\left(t_{0}\right) \omega_{1}(t)+\left|\begin{array}{cc}
\omega_{1} & \omega_{2} \\
\dot{\omega}_{1} & \dot{\omega}_{2}
\end{array}\right| \omega_{4}
$$

Assim

$$
\omega_{2}(+\infty)=\omega_{2}\left(t_{0}\right) \omega_{1}(+\infty)+\left|\begin{array}{cc}
\omega_{1} & \omega_{2} \\
\dot{\omega}_{1} & \dot{\omega}_{2}
\end{array}\right| \omega_{4}(+\infty)
$$

Mas $\omega_{1}(+\infty)=0$ e $\omega_{4}(+\infty)>1$ daí

$$
\operatorname{sgn}\left(\dot{\omega}_{2}(\infty)\right)=\left|\begin{array}{ll}
\omega_{1} & \omega_{2} \\
& \\
\dot{\omega}_{1} & \dot{\omega}_{2}
\end{array}\right|
$$

Isso conclui a demonstração do lema. 
Lema 4.7. Suponha que em uma vizinhança de um ponto $\left(v_{0}, \tau_{0}\right) \in \Pi_{0}^{+}$estão definidos campos contínuos de condições iniciais

$$
\delta_{i}=\left\{\omega_{0, i}(v, \tau), \dot{\omega}_{0, i}(v, \tau)\right\}, \quad i=1,2
$$

e seja $\omega_{i}(t, v, \tau)$ solução de (104) com condições iniciais (105) são dadas por $\delta_{i}$. Se

1) $\delta_{1} h^{+}=0$ e $\delta_{1} T^{+}<0$ em $R_{0}^{+} e$

2) $\left|\begin{array}{ll}\omega_{0,1} & \omega_{0,1} \\ \dot{\omega}_{0,1} & \dot{\omega}_{0,2}\end{array}\right|_{\left(v_{0}, \tau_{0}\right)}>0$

então existe uma vizinhança $U$ de $\left(v_{0}, \tau_{0}\right), t^{*}$ e $C>0$ tal que

$$
\dot{\omega}_{2}(t, v, \tau) \geq C>0
$$

para todo $(v, \tau) \in U \cap R_{0}^{+}$. O lema é verdadeiro trocando-se + por - .

Demonstração. Demonstraremos o lema apenas no caso das funções que levam o símbolo + . Desde que $\left(v_{0}, \tau_{0}\right) \in \Pi_{0}^{+}$, temos que a solução correspondente, $z\left(t, v_{0}, \tau_{0}\right)$ é parabólica e então, para $v$ grande $\dot{z} \geq \frac{v}{2}$.

Daí existe uma vizinhança $V$ do ponto $\left(v_{0}, \tau_{0}\right)$ e um número $t_{0} \geq \sup _{\tau \in V} \tau$ tal que para $(v, \tau) \in$ $V \cap \mathbb{R}_{0}^{+}$temos que $z\left(t_{0}, v, \tau\right) \geq z^{*}$, e sobre o intervalo $\left[\tau, t_{0}\right], \dot{z}>0$. Aqui $z^{*}$ é o número definido em $6^{\circ}$.

Para $(v, \tau) \in V \cap\left(R_{0}^{+} \cap \Pi_{0}^{+}\right)$e $t_{0} \leq t \leq T^{+}(v, \tau)$ a função $p(t, v, \tau)$ é não negativa, pois

$$
Q_{z}^{\prime}(z, t) \leq 0, \forall z \geq z^{*}
$$

Em $R_{0}^{+}$as equações (103) e (104) são idênticas, para ver isto basta substituir $\omega$ por $\sigma z$. A derivada $\delta_{i} z(t, v, \tau)$ que é solução de (103) coincide com a solução $\omega_{i}(t, v, \tau)$.

Por hipótese temos que

$$
\omega_{1}\left(T^{+}(v, \tau), v, \tau\right)=\delta_{1} z\left(T^{+}(v, \tau), v, \tau\right)=\frac{\delta_{1} h^{+}(v, \tau)}{Q_{0}\left(Z^{+}\right)}=0
$$

e aplicando o Teorema de Schwarz temos ainda que 


$$
\dot{\omega}_{1}\left(T^{+}(v, \tau), v, \tau\right)=\delta_{1} \dot{z}\left(T^{+}(v, \tau), v, \tau\right)=Q\left(Z^{+}(v, \tau), T^{+}(v, \tau)\right) \delta_{1} T^{+}(v, \tau)<0
$$

Ambas as equações vem de (77) e do fato de que em $R_{0}^{+}$

$$
h^{+}(v, \tau)=\int_{0}^{Z^{+}(v, \tau)} Q_{0}(z) d z
$$

Sobre o intervalo $\left[t_{0}, T^{+}\right]$a função $p \geq 0$ e dessa forma, usando o lema anterior, a solução quando muito tem um zero. Então por (108) e (109) implicam que $\omega_{1}$ é positiva para

$$
t_{0} \leq t \leq T^{+}
$$

mas

$$
\ddot{\omega}_{1}=p \omega_{1} \leq 0 \text {. }
$$

Daí $\dot{\omega}_{1}$ é não decrescente

$$
\omega_{1}(t, v, \tau), \dot{\omega}_{1}(t, v, \tau)<0
$$

$\mathrm{O}$ ponto $\left(v_{0}, \tau_{0}\right) \in \Pi_{0}^{+}$e quando $(v, \tau) \rightarrow\left(v_{0}, \tau_{0}\right)$ implica que $Z^{+}(v, \tau) \rightarrow+\infty$ e daí temos que $T^{+}(v, \tau) \rightarrow+\infty$.

Quando passamos o limite em (110) para $t_{0} \leq t<+\infty$ encontramos

$$
\omega_{1}\left(t, v_{0}, \tau_{0}\right) \geq 0, \quad \dot{\omega}_{1}\left(t, v_{0}, \tau_{0}\right) \leq 0
$$

Sobre o ponto $\left(v_{0}, \tau_{0}\right)$ a equação (104) satisfaz as hipóteses do lema anterior, e então existe $\omega_{1}\left(+\infty, v_{0}, \tau_{0}\right)$. Por $(111)$ este limite não pode ser positivo e nem negativo, pois $\omega_{1} \geq 0$. Assim $\omega_{1}(+\infty)=0$.

Se aplicarmos o lema anterior novamente, encontramos

$$
\dot{\omega}_{2}\left(+\infty, v_{0}, \tau_{0}\right)=\lim _{t \rightarrow+\infty} \dot{\omega}_{2}\left(t, v_{0}, \tau_{0}\right)>0,
$$

já que o Wronskiano é positivo e constante em $t$.

Se $t^{*} \geq t_{0}$ tal que 


$$
\omega_{2}\left(t^{*}, v, \tau\right)>0, \quad \dot{\omega}_{2}\left(t^{*}, v, \tau\right)>C=\frac{1}{2} \dot{\omega}\left(t^{*}, v_{0}, \tau_{0}\right)
$$

Isso segue da dependência contínua da solução $\omega_{2} \operatorname{com}$ respeito às entradas $(v, \tau)$.

Seja $\left[t^{*}, t^{* *}\right)$ o subintervalo maximal em $\left[t^{*}, T^{+}\right)$tal que $\dot{\omega}_{2}>0$. Nesse subintervalo

$$
\omega_{2}(t, v, \tau)>\omega_{2}\left(t^{*}, v, \tau\right)>0^{\prime}, \quad \ddot{\omega}_{2}=p \omega_{2}
$$

Assim, $\dot{\omega}_{2}$ é não decrescente no intervalo $\left[t^{*}, T^{+}\right)$.

Introduziremos agora a mudança de variáveis

$$
\xi=h^{-}(v, \tau), \quad \eta=h^{+}(v, \tau)
$$

Nessas novas coordenadas a aplicação $S: R_{0}^{+} \rightarrow R_{0}^{-}$, tal que $S(v, \tau)=\left(v^{\prime}, \tau^{\prime}\right)$ toma a seguinte forma

$$
S(\xi, \eta)=S\left(h^{-}(v, \tau), h^{+}(v, \tau)\right)=S\left(h^{-}\left(v^{\prime}, \tau^{\prime}\right), h^{+}\left(v^{\prime}, \tau^{\prime}\right)\right)
$$

e pela proposição 4.4 temos

$$
S(\xi, \eta)=(\eta, f(\xi, \eta))
$$

estudaremos se tal mudança de coordenadas (112) é regular, isto é, se

$$
J(v, \tau)=\frac{\partial(\xi, \eta)}{\partial(v, \tau)} \neq 0
$$

no caso em que $(v, \tau) \in \Pi_{0}^{+} \cap \Pi_{0}^{-}$quando $\Im$ é finito.

Proposição 4.3. Se $J\left(v_{0}, \tau_{0}\right) \neq 0$ sobre $\left(v_{0}, \tau_{0}\right)$ em $\Pi_{0}^{+}$então para alguma constante $C>0$ quando $(v, \tau) \rightarrow\left(v_{0}, \tau_{0}\right)$ em $R_{0}^{+}$temos

$$
\frac{\partial T}{\partial \xi}^{+} \rightarrow \frac{v}{J}, \quad \frac{\partial T^{+}}{\partial \eta}+\frac{\partial T^{+}}{\partial \xi} \geq \frac{C}{v Q_{0}\left(X^{+}(v, \tau)\right)}
$$

Uma proposição análoga pode ser enunciada para o caso $\left(v_{0}, \tau_{0}\right) \in R_{0}^{-}$para isso tomamos o seguinte cuidado de verificar que existe $C>0$ tal que

$$
\frac{\partial T^{-}}{\partial \eta} \rightarrow \frac{v}{J}, \frac{\partial T^{-}}{\partial \eta}+\frac{\partial T^{-}}{\partial \xi} \leq-\frac{C}{v Q_{0}\left(X^{-}(v, \tau)\right)}
$$


Demonstração. Estamos supondo que $J\left(v_{0}, \tau_{0}\right) \neq 0$ numva vizinhnaça $V$ de $\left(v_{0}, \tau_{0}\right)$ assim, existe uma mudança de coordenadas $\sigma^{-1}: V \rightarrow \sigma(V)$ tal que

$$
\sigma^{-1}(v, \tau)=(\xi, \eta) \Rightarrow \sigma(\xi, \eta)=(v, \tau)
$$

Assim, a partir desta mudança de coordenadas, podemos definir localmente as seguintes funções:

$$
\begin{aligned}
& Z^{ \pm}(\xi, \eta)=Z^{ \pm}(\sigma(\xi, \eta)) \\
& T^{ \pm}(\xi, \eta)=T^{ \pm}(\sigma(\xi, \eta))
\end{aligned}
$$

e a solução

$$
z(t, \xi, \eta)=z(t, \sigma(\xi, \eta))=z(t, v, \tau)
$$

procedendo desta maneira temos que

$$
\begin{gathered}
z\left(T^{ \pm}(\xi, \eta), \xi, \eta\right)=Z^{ \pm}(\xi, \eta) \\
\dot{z}\left(T^{ \pm}(\xi, \eta), \xi, \eta\right)=0
\end{gathered}
$$

a partir desta duas equações obtemos os análogos às equações dada em (77)

Temos então que

$$
\left({\frac{\partial T^{+}}{\partial \xi}}^{+} \frac{\partial T^{+}}{\partial \eta}\right) \equiv \frac{1}{Q\left(Z^{+}, T^{+}\right)}\left(\dot{z}_{\xi}\left(T^{+}\right), \dot{z}_{\eta}\left(T^{+}\right)\right)
$$

Por simplicidade, de agora em diante, consideraremos as coordenadas $(\xi, \eta)$ e omitiremos o símbolo + das funções envolvidas, como $T^{+}$e $Z^{+}$.

O determinante Wronskiano para o par de soluções $z_{\xi}, z_{\eta}$ da equação (103), nas coordenadas $(\xi, \eta)$, é constante. Se calcularmos este para o tempo $t=\tau$ nós podemos encontrar $\dot{z}_{\xi}$. De fato

$$
\delta z(T)=\delta Z(T)=\frac{\delta h^{+}}{Q_{0}(Z)}=\frac{\delta \eta}{Q_{0}(Z)}
$$

e dessa forma temos que 


$$
z_{\xi}(T)=0, \quad z_{\eta}(T)=\frac{1}{Q_{0}(Z)}
$$

Para o tempo $t=\tau$ temos que

$\left|\begin{array}{cc}0 & \frac{1}{Q_{0}(Z)} \\ \dot{z}_{\xi}(T) & \dot{z}_{\eta}(T)\end{array}\right|=\left|\begin{array}{cc}z_{\xi} & z_{\eta} \\ \dot{z}_{\xi} & \dot{z}_{\eta}\end{array}\right|_{t=\tau}=\left|\begin{array}{cc}-v \tau_{\xi} & -v \tau_{\eta} \\ v_{\xi} & v_{\eta}\end{array}\right|=-v \frac{\partial(\tau, v)}{\partial(\xi, \eta)}=v \frac{\partial(v, \tau)}{\partial(\xi, \eta)}=v \frac{1}{J(v, \tau)}$

isso vem do fato de que

$$
z(\tau(\xi, \eta), \xi, \eta) \equiv 0 \Rightarrow \dot{z}(\tau, \xi, \eta) \tau_{\xi}+z_{\xi}(\tau, \xi, \eta)=v \tau_{\xi}+z_{\xi}(\tau, \xi, \eta)=0
$$

o mesmo ocorre trocando-se $\xi$ por $\eta$

Ainda temos também que

$$
v(\xi, \eta) \equiv \dot{z}(\tau, \xi, \eta)
$$

Dessa forma, do primeiro dos determinantes de (117) segue que

$$
\frac{\dot{z}_{\xi}}{Q_{0}(Z)}=-\frac{v}{J(v, \tau)} \Rightarrow \dot{z}_{\xi}(T)=-\frac{v Q_{0}(Z)}{J(v, \tau)}
$$

e então

$$
\frac{\partial T}{\partial \xi}=\frac{\dot{z}_{\xi}}{Q(Z)}=-\frac{v Q_{0}(Z)}{J(v, \tau) Q(Z, T)}
$$

mas o limite

$$
\lim _{(v, \tau) \in R_{0}^{+} \rightarrow\left(v_{0}, \tau_{0}\right)} \frac{Q_{0}(Z)}{Q(Z, T)}=1
$$

De fato

$$
\left|\frac{Q_{0}(Z)}{Q(Z, T)}-1\right|=\left|\frac{Q_{0}(Z)-Q(Z, T)}{Q(Z, T)}\right| \leq \frac{2 \pi \psi(Z)}{q(Z)} \leq 2 \pi \xi(Z) q(Z)
$$

conforme as condições $3^{\circ o}, 5^{\circ}$ e lema 2.1 .

Sabemos que $Q_{0}(z) \geq q(z)>0$ para todo $z>0$

$$
Q_{0}(z)=\frac{1}{2 \pi} \int_{0}^{2 \pi} Q(z, t) d t \Rightarrow \frac{\partial Q_{0}(z)}{\partial z}=\frac{1}{2 \pi} \int_{0}^{2 \pi} \frac{\partial Q}{\partial z}(z, t) d t<0, \forall z \geq z^{*}
$$


Assim $Q_{0}$ é decrescente para $z \geq z^{*}$. Como $\Im=\int_{0}^{+\infty} Q_{0}(z) d z$ é finito então temos que

$$
\lim _{z \rightarrow+\infty} Q_{0}(z)=0
$$

Como

$$
0<q(z) \leq Q_{0}(z)
$$

implica que

$$
\lim _{z \rightarrow+\infty} q(z)=0
$$

Daí quando $(v, \tau) \rightarrow\left(v_{0}, \tau_{0}\right)$ temos que $Z(v, \tau) \rightarrow+\infty$ logo

$$
\lim _{(v, \tau) \rightarrow\left(v_{0}, \tau_{0}\right)}\left|\frac{Q_{0}(Z)}{Q(Z, T)}-1\right|=0
$$

Para estimar $\dot{z}_{\eta}$ usaremos o lema anterior. Os dois campos de condições iniciais no lema são definidos por operadores diferenciais. Seja o seguinte campo

$$
\left\{\begin{array}{l}
\delta_{1}=\frac{J(v, \tau)}{v^{2}} \frac{\partial}{\partial \xi} \\
\delta_{2}=v \frac{\partial}{\partial \eta}
\end{array}\right.
$$

Este é um campo contínuo e além disso temos

$$
\delta_{1} h^{+}=\frac{J(v, \tau)}{v^{2}} \frac{\partial h^{+}}{\partial \xi}=\frac{J(v, \tau)}{v^{2}} \frac{\partial \eta}{\partial \xi}=0
$$

e

$$
\delta_{1} T=\frac{J(v, \tau)}{v^{2}} \frac{\partial T}{\partial \xi}=-\frac{J}{v^{2}} \frac{v Q_{0}}{J Q}=-\frac{Q_{0}(Z)}{v Q(Z)}<0
$$

Vamos supor que $J(v, \tau)>0$. Disso e da expressão acima, todas as hipóteses do lema anterior são satisfeitas. Assim, existe uma vizinhança $V$ de $\left(v_{0}, \tau_{0}\right)$ e números reais $t^{*}$ e $C>0$ tais que para $(v, \tau) \in R_{0}^{+} \cap V$

$$
\omega_{2}(t, v, \tau) \geq 2 C>0
$$

para todo $t^{*} \leq t \leq T^{+}(v, \tau)$

Logo temos que 


$$
\frac{\partial T}{\partial \eta}=\frac{\dot{z}_{\eta}}{Q(Z, T)}=\frac{\delta_{2} \dot{z}}{v Q(Z, T)}=\frac{\omega_{2}(T)}{v Q(Z, T)} \geq \frac{2 C}{v Q(Z, T)}
$$

De equações (118) e (129) temos que

$$
\frac{\partial T}{\partial \eta}+\frac{\partial T}{\partial \eta} \geq \frac{2 C}{v Q(Z, T)}-\frac{v Q_{0}(Z)}{J(v, \tau) Q(Z, T)}=\frac{1}{v Q(Z, T)}\left[2 C-\frac{v^{2} Q_{0}(Z)}{J}\right] \geq \frac{C}{v Q_{0}(Z)}
$$

Visto que quando $(v, \tau) \sim\left(v_{0}, \tau_{0}\right)$ temos que $Q(Z, T) \sim Q_{0}(Z)$ e

$$
\lim _{(v, \tau) \in R_{0}^{+} \rightarrow\left(v_{0}, \tau_{0}\right)} Q_{0}(Z)=0
$$

Lembrando que nesta proposição, $Z$ e $T$ indicam $Z^{+}$e $T^{+}$respectivamente.

Temos assim concluído a demonstração desta proposição.

Para provar esta proposição para os análogos $T^{-}$e $Z^{-}$devemos observar que

$$
\delta z\left(T^{-}\right)=-\delta\left(Z^{-}\right)=-\frac{\delta h^{-}}{Q_{0}\left(Z^{-}\right)}=-\frac{\delta \xi}{Q_{0}\left(Z^{-}\right)}
$$

e com isso

$$
z_{\xi}\left(T^{-}\left(v^{\prime}, \tau^{\prime}\right)\right)=-\frac{1}{Q_{0}\left(Z^{-}\left(v^{\prime}, \tau^{\prime}\right)\right)} z_{\eta}\left(T^{-}\left(v^{\prime}, \tau^{\prime}\right)\right)=0
$$

daí o determinate análogo em (117) resulata no valor $\frac{v^{\prime}}{J\left(v^{\prime}, \tau^{\prime}\right)}$.

Prosseguimos a definição de maneira análoga e depois definimos o campo

$$
\left\{\begin{array}{l}
\delta_{1}=-\frac{J\left(v^{\prime}, \tau^{\prime}\right)}{v^{\prime 2}} \frac{\partial}{\partial \eta} \\
\delta_{2}=-v^{\prime} \frac{\partial}{\partial \xi}
\end{array}\right.
$$

Podemos fazer isso pois $-z(t, v, \tau)$ é solução de $(14)$.

Uma outra observação que devemos fazer é que podemos supor sem perda de generalidade que $J>0$, caso contrário, redefinimos $J$ da seguinte maneira

$$
J(v, \tau)=\frac{\partial(\eta, \xi)}{\partial(v, \tau)}
$$

e assim a proposição 4.3 fica verdadeira, desde que troquemos $T^{+}$por $T^{-}$e vice-versa. 
Proposição 4.4. Sejam $U_{1}$ e $U_{2}$ vizinhanças de pontos $\left(v_{1}, \tau_{1}\right) \in \Pi_{0}^{+}$e $\left(v_{2}, \tau_{2}\right) \in \Pi_{0}^{-}$. Se $J\left(v_{i}, \tau_{i}\right) \neq 0$ para $i=1,2$ então quando $U_{i} \rightarrow\left(v_{i}, \tau_{i}\right)$, na interseção $U_{1} \cap S^{-1}\left(U_{2} \cap R_{0}^{-}\right)$ temos

$$
d S(\xi, \eta)=\left(\begin{array}{cc}
0 & 1 \\
-O(1) & \frac{\alpha J\left(v^{\prime}, \tau^{\prime}\right)}{v^{2} Q_{0}\left(X^{+}(v, \tau)\right)}+1-O(1)
\end{array}\right)
$$

onde $O(1)$ é uma função nas variáveis $v, \tau$ que satisfaz

$$
\lim _{(v, \tau) \in R_{0}^{+} \rightarrow\left(v_{1}, \tau_{1}\right)} O(1)=1
$$

tal que $\alpha=\alpha(v, \tau)>C>0$.

Demonstração. Primeiramente lembremos que

$$
\begin{gathered}
P^{+}(v, \tau)=\left(h^{+}(v, \tau), T^{+}(v, \tau)\right)=\left(\eta, T^{-}\left(v^{\prime}, \tau^{\prime}\right)\right)=\left(\xi^{\prime}, T^{-}\left(v^{\prime} \tau^{\prime}\right)\right) \\
P^{-}\left(v^{\prime}, \tau^{\prime}\right)=\left(v^{\prime}, \tau^{\prime}\right)=S(v, \tau) \Rightarrow \sigma^{-}\left(v^{\prime}, \tau^{\prime}\right)=\left(\xi^{\prime}, \eta^{\prime}\right)
\end{gathered}
$$

Assim temos que

$$
\begin{gathered}
\sigma^{-1} \circ\left(P^{-}\right)^{-1} \circ P^{+}(v, \tau=\sigma(\xi, \eta))=\left(\xi^{\prime}, \eta^{\prime}\right) \\
\left.\sigma^{-1} \circ\left(P^{-}\right)^{-1} \circ P^{+} \circ \sigma(\xi, \eta)\right)=\left(\xi^{\prime}, \eta^{\prime}\right)
\end{gathered}
$$

$\operatorname{como}\left(P^{-}\right)^{-1} \circ P^{+}=S$

temos que

$$
\sigma^{-1} \circ S \circ \sigma(\xi, \eta)=\left(\xi^{\prime}, \eta^{\prime}\right)
$$

Se chamarmos de $\widetilde{S}$ a aplicação, tal que

$$
\widetilde{S}(\xi, \eta)=\left(\xi^{\prime}, \eta\right)=(\eta, f(\xi, \eta))
$$

temos que 


$$
\widetilde{S}=\sigma^{-1} \circ S \circ \sigma
$$

Vemos então que localmente, a mudança de coordenadas $\sigma$ é uma conjuação entre as dinâmicas $S$ e $\widetilde{S}$. Da mesma maneira, podemos denotar

$$
\begin{aligned}
& \widetilde{P}^{+}=\sigma^{-1} \circ P^{+} \sigma \\
& \widetilde{P}^{-}=\sigma^{-1} \circ P^{+} \sigma
\end{aligned}
$$

e temos que

$$
\widetilde{S}=\left(\widetilde{P}^{-}\right)^{-1} \circ \widetilde{P}^{+}
$$

O resultado desta proposição diz respeito ao diferencial de $\widetilde{S}$, para não carregarmos a notação denotaremos apenas por $S$, assim como trataremos $\widetilde{P}^{ \pm}$apenas por $P^{ \pm}$para não carregar a notação. Trabalharemos nas coordenadas $(\xi, \eta)$.

Da proposição anterior temos que

$$
\begin{aligned}
& d S=\left(d P^{-}\right)_{\left(\xi^{\prime}, \eta^{\prime}\right)}^{-1} \cdot d P_{(\xi, \eta)}^{+}= \\
& \left(\left[\begin{array}{cc}
1 & 0 \\
\frac{\partial T^{-}}{\partial \xi} & \frac{\partial T^{-}}{\partial \eta}
\end{array}\right]_{\left(\xi^{\prime}, \eta^{\prime}\right)}\right]^{-1} \cdot\left[\begin{array}{cc}
1 & 0 \\
\frac{\partial T^{+}}{\partial \xi} & \frac{\partial T^{+}}{\partial \eta}
\end{array}\right]_{(\xi, \eta)}
\end{aligned}
$$

Calculundo a matriz inversa que aparece em (121) temos

$$
d\left(P^{-}\right)^{-1}=\left[\begin{array}{cc}
1 & 0 \\
-\frac{\frac{\partial T}{\partial \xi}^{-}}{\frac{\partial t}{\partial \eta}^{-}} & \frac{1}{\frac{\partial T}{\partial \eta}^{-}}
\end{array}\right]
$$

Substituindo em (121) temos que 


$$
d S=\left[\begin{array}{cc}
0 & 1 \\
\frac{\frac{\partial T}{\partial \xi}}{\frac{\partial T^{-}}{\partial \eta^{\prime}}} & \frac{{\frac{\partial T}{\partial \eta^{\prime}}}^{+}-\frac{\partial T}{\partial \xi^{\prime}}}{\frac{\partial T}{\partial \eta^{\prime}}}
\end{array}\right]
$$

Estamos empregando a seguinte notação em (122)

$$
{\frac{\partial T^{-}}{\partial \xi^{\prime}}}^{-}=\frac{\partial T^{-}}{\partial \xi}\left(\xi^{\prime}, \eta^{\prime}\right)
$$

Já sabemos do capítulo 2 que $Z^{+}(v, \tau)=Z^{-}\left(v^{\prime}, \tau^{\prime}\right)$ e que $T^{+}(v, \tau)=T^{-}\left(v^{\prime}, \tau\right)^{\prime}$ e usando (115) e (116) na vizinhança $V=U_{1} \cap S^{-1}\left(U_{2} \cap R_{0}^{-}\right)$temos que

$$
\frac{\frac{\partial T}{\partial \xi}^{+}}{{\frac{\partial T}{\partial \eta^{\prime}}}^{-}}=-\frac{\frac{v Q_{0}\left(Z^{+}(v, \tau)\right)}{Q\left(Z^{+}(v, \tau), T^{+}(v, \tau)\right) J(v, \tau)}}{\frac{v^{\prime} Q_{0}\left(Z^{-}\left(v^{\prime}, \tau^{\prime}\right)\right)}{Q\left(Z^{-}\left(v^{\prime}, \tau^{\prime}\right)\right) J\left(v^{\prime}, \tau^{\prime}\right)}}=-\frac{v J\left(v^{\prime}, \tau^{\prime}\right)}{v^{\prime} J(v, \tau)}=-O(1)
$$

o quociente acima, dado em (123) converge a -1 quando $U_{1}$ se torna pequena até convergir ao ponto $\left(v_{1}, \tau_{1}\right)$, isso segue da continuidade das funções envolvidas no quociente acima.

De acordo com a porposição anterior, das equações (115) e (116) temos que

$$
\begin{gathered}
\frac{\partial T^{+}}{\partial \xi}+\frac{\partial T^{+}}{\partial \eta} \geq \frac{C}{v Q_{0}\left(Z^{+}(v, \tau)\right)} \\
-\frac{\partial T^{-}}{\partial \xi^{\prime}}-\frac{\partial T^{-}}{\partial \eta^{\prime}} \geq \frac{C}{v^{\prime} Q_{0}\left(Z^{-}\left(v^{\prime}, \tau^{\prime}\right)\right)}=\frac{C \frac{v}{v^{\prime}}}{v Q_{0}\left(Z^{+}(v, \tau)\right)}
\end{gathered}
$$

Somando essas duas expressões temos o seguinte

$$
\left({\frac{\partial T^{+}}{\partial \eta}}^{+}-\frac{\partial T^{-}}{\partial \xi^{\prime}}\right)+\left({\frac{\partial T^{+}}{\partial \xi}}^{+}-\frac{\partial T^{-}}{\partial \eta^{\prime}}\right) \geq \frac{C\left(1+\frac{v}{v^{\prime}}\right)}{v Q_{0}\left(Z^{+}(v, \tau)\right)}=\frac{\alpha_{1}}{v Q_{0}\left(Z^{+}(v, \tau)\right)}, \alpha_{1} \geq C\left(1+\frac{v}{v^{\prime}}\right)>C
$$

Assim podemos escrever

$$
\left({\frac{\partial T^{+}}{\partial \eta}}^{+}-\frac{\partial T^{-}}{\partial \xi^{\prime}}\right)+\left({\frac{\partial T^{+}}{\partial \xi}}^{+}-\frac{\partial T^{-}}{\partial \eta^{\prime}}\right)=\frac{\alpha_{0}(v, \tau)}{v Q_{0}\left(Z^{+}(v, \tau)\right)}
$$

Disso segue que

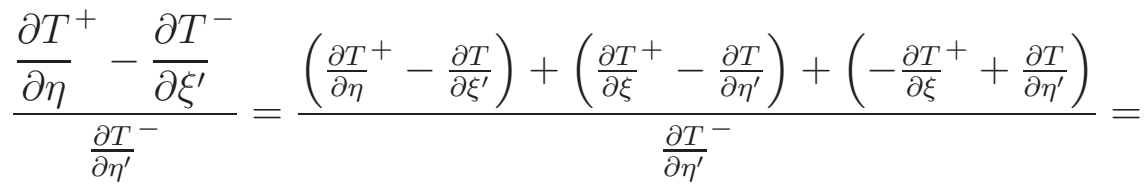




$$
\frac{\alpha_{0}(v, \tau)}{\frac{v^{\prime}}{J\left(v^{\prime}, \tau^{\prime}\right)} v Q_{0}\left(Z^{+}(v, \tau)\right)}+1-\frac{\frac{\partial T}{\partial \xi}^{+}}{{\frac{\partial T}{\partial \eta^{\prime}}}^{-}}=\frac{\alpha_{0}(v, \tau) J\left(v^{\prime}, \tau^{\prime}\right) \frac{v}{v^{\prime}}}{v^{2} Q_{0}\left(Z^{+}(v, \tau)\right)}+1-O(1)
$$

Ponha

$$
\alpha(v, \tau)=\alpha_{0}(v, \tau) \frac{v}{v^{\prime}}
$$

Das equações (123) e (124), substituindo-as em (122) segue o resultado da proposição.

Proposição 4.5. O diferencial dS verifica as condições de i)a iii) do capítulo 1 e as hipóteses dos teoremas 1.3 e 1.4 .

Demonstração. Segundo (113) vamos considerar

$$
S(\xi, \eta)=(\eta, f(\xi, \eta))
$$

e pela proposição 4.4 temos que

$$
d S(\xi, \eta)=\left(\begin{array}{cc}
0 & 1 \\
-O(1) & \frac{\alpha J\left(v^{\prime}, \tau^{\prime}\right)}{v^{2} Q_{0}\left(X^{+}(v, \tau)\right)}+1-O(1)
\end{array}\right)=\left(\begin{array}{cc}
0 & 1 \\
& \\
f_{\xi} & f_{\eta}
\end{array}\right)
$$

Observemos que o determinante

$$
\Delta=\left|\begin{array}{cc}
0 & 1 \\
f_{\xi} & f_{\eta}
\end{array}\right|=f_{\xi} \approx 1
$$

quando $\xi$ e $\eta$ estão ambos próximos a $\Im$. Além disso quando $\xi$ e $\eta$ ficam muito próximos de $\Im$ temos que

$$
\left|f_{\eta}\right| \rightarrow+\infty
$$

Podemos então escolher uma vizinhança de $(\Im, \Im)$ tal que

$$
\frac{1}{2}<\left|f_{\xi}\right|<2, \quad\left|f_{\eta}\right|>M>6, \frac{\left|f_{\eta}\right|}{\left|f_{\xi}\right|}>M>6
$$

Chame de $C^{+}=C_{1 / 4}^{u}=\left\{(x, y) \in \mathbb{R}^{2} ; \quad|y| \geq 4|x|\right\}$

Mostremos que $d S\left(C^{+}\right) \subset C^{+}$. Seja $(x, y) \in C^{+}$, vamos sempre considerar $y \neq 0$ pois $y=0$ implica $x=0$, pela definição de $C^{+}$. 


$$
d S(x, y)=\left(\begin{array}{cc}
0 & 1 \\
f_{\xi} & f_{\eta}
\end{array}\right)\left(\begin{array}{l}
x \\
y
\end{array}\right)=\left(\begin{array}{c}
y \\
\\
f_{\xi} x+f_{\eta} y
\end{array}\right)=\left(\begin{array}{c}
x^{\prime} \\
\\
y^{\prime}
\end{array}\right)
$$

Se $x=0$

$$
\left|f_{\xi} x+f_{\eta} y\right|>0
$$

Agora se $x \neq 0$

$$
\left|f_{\xi} x+f_{\eta} y\right|>0 \geq\left|f_{\eta} y\right|-\left|f_{\xi} x\right|>6|x|-2|x|=4|x|>0
$$

Assim temos

$$
\frac{\left|y^{\prime}\right|}{\left|x^{\prime}\right|} \geq \frac{\left|f_{\eta}\right||y|-\left|f_{\xi}\right||x|}{|y|}=\left|f_{\eta}\right|-\left|f_{\xi}\right| \frac{|x|}{|y|} \geq 6-\frac{1}{4}\left|f_{\xi}\right|>4
$$

Disso temos que $d S\left(C^{+}\right) \subset C^{+}$e temos ainda que $\left|x^{\prime}\right|=|y| \leq 4|x|$.

Observemos que

$$
\max \{|x|,|y|\}=|y|, \quad \max \left\{\left|x^{\prime}\right|,\left|y^{\prime}\right|\right\}=\left|y^{\prime}\right|
$$

e como já sabemos

$$
\frac{\left|y^{\prime}\right|}{|y|} \geq 4
$$

Portanto

$$
\|d S(x, y)\| \geq 4\|(x, y)\|,(x, y) \in C^{+}
$$

Agora, denotemos

$$
C^{-}=C_{1 / 4}^{s}=\left\{(x, y) \in \mathbb{R}^{2} ;|x| \geq 4|y|\right\}
$$

Da expressão (125), podemos calcular a inversa de $d S$ para obter 


$$
d S^{-1}=\left(\begin{array}{cc}
-\frac{f_{\eta}}{f_{\xi}} & \frac{1}{f_{\xi}} \\
1 & 0
\end{array}\right)
$$

Assim, para $(x, y) \in C^{-}$temos

$$
d S^{-1}(x, y)=\left(\begin{array}{cc}
0 & 1 \\
f_{\xi} & f_{\eta}
\end{array}\right)\left(\begin{array}{l}
x \\
y
\end{array}\right)=\left(\begin{array}{c}
\frac{1}{f_{\xi}}\left(-f_{\eta} x+y\right) \\
x
\end{array}\right)=\left(\begin{array}{c}
x^{\prime} \\
\\
y^{\prime}
\end{array}\right)
$$

Como fizemos anteriormente, consideraremos apenas o caso em que $x \neq 0$.

$$
\frac{\left|x^{\prime}\right|}{\left|y^{\prime}\right|}=\frac{\left|f_{\eta} x-y\right|}{\left|f_{\xi} x\right|} \geq \frac{\left|f_{\eta}\right|}{\left|f_{\xi}\right|}-\frac{|y|}{\left|f_{\xi} x\right|} \geq \frac{\left|f_{\eta}\right|}{\left|f_{\xi}\right|}-2 \frac{1}{4}>M-\frac{1}{2}>M-2>4
$$

Disso segue que $\left|x^{\prime}\right|>4\left|y^{\prime}\right|$ e isso implica que

$$
d S^{-1}\left(C^{-}\right) \subset C^{-}
$$

Desde que $(x, y) \in C^{-}$e $d S^{-1}\left(C^{-}\right) \subset C^{-}$temos que

$$
|(x, y)|=x, \quad\left|\left(x^{\prime}, y^{\prime}\right)\right|=\left|x^{\prime}\right|
$$

Como $y^{\prime}=y$, segue que

$$
\left|d S^{-1}(x, y)\right| \geq 4|(x, y)|
$$

para $(x, y) \in C^{-}$.

Definição 4.1. Um ponto $p=(v, \tau) \in \Pi_{0}^{+} \cap \Pi_{0}^{-}$é chamado de regular se

$$
J(v, \tau)=\frac{\partial\left(h^{-}, h^{+}\right)}{\partial(v, \tau)} \neq 0 .
$$

Verificaremos as condições que garantem que $J(v, \tau) \neq 0 \operatorname{com}(v, \tau) \in \Pi_{0}^{+} \cap \Pi_{0}^{-}$. A verificação de tal fato segue nos resultados dados a seguir.

Proposição 4.6. Se as funções contínuas

$$
Q(z, t, \varepsilon)=Q(z)+\varepsilon \widetilde{Q}(z, t, \varepsilon)
$$


satisfazem as condições $1^{\circ}, 2^{\circ}$, e $3^{o o}$ com respeito a $(z, t)$ e $5^{o}$ uniformemente em $\varepsilon$, trocando-se em $4^{o} \psi$ por $\varepsilon \psi, \widetilde{Q}(z, t, \varepsilon)$ analítica em $\varepsilon$ e $\int_{0}^{+\infty} Q_{0}(z, \varepsilon) d z$ é uniformemente limitada em $\varepsilon$, então quando $\varepsilon \rightarrow 0$

$$
\begin{gathered}
h^{ \pm}(v, \tau)=\frac{v^{2}}{2}+\varepsilon \int_{0}^{X(v)}\left[Q_{1,0}(y)-Q_{1}\left(y, t^{ \pm}(y, \tau)\right)\right] d y+O(\varepsilon) \\
\frac{\partial h^{ \pm}}{\partial v}=v+O(\varepsilon)
\end{gathered}
$$

$$
\frac{\partial h^{ \pm}}{\partial \tau}=\varepsilon \int_{0}^{X^{v}} Q_{1, t}^{\prime}\left(y, t^{ \pm}(y, \tau)\right) d y+o(\varepsilon)
$$

onde

$$
\begin{gathered}
Q_{0}(z, \varepsilon)=\frac{1}{2 \pi} \int_{0}^{2 \pi} Q(z, t, \varepsilon) d t, \quad Q_{1}(x, t)=\widetilde{Q}(z, t, 0) \\
Q_{10}(z)=\frac{1}{2 \pi} \int_{0}^{2 \pi} Q_{1}(z, t), \quad t^{ \pm}(v, \tau)=\tau+ \pm \int \frac{d \sigma}{V(\sigma)}
\end{gathered}
$$

$V^{2}(\sigma)=v^{2}-2 \int_{0}^{\sigma} Q(z) d z$ e $X(v)$ é obtido a partir de $\frac{v^{2}}{2}=\int_{0}^{X(v)} Q(z) d z$ se $\frac{v^{2}}{2} \leq$ $\int_{0}^{+\infty} Q(z) d z$ e caso contrário definiremos $X(v)=+\infty$. Os restos em $(127)-(129)$ são uniformes em cada subconjunto compacto de $\Phi$.

Demonstração. Vamos considerar funções $h^{ \pm}(y, v, \tau, \varepsilon)$ e $t^{ \pm}(y, v, \tau, \varepsilon)$ satisfazendo sistemas

$$
\left\{\begin{array}{l}
\frac{\partial h^{ \pm}}{\partial y}=Q_{0}(y, \varepsilon)-Q\left(y, t^{ \pm}\right),\left.\quad h^{ \pm}\right|_{y=0}=\frac{v^{2}}{2} \\
\frac{\partial t^{ \pm}}{\partial y}= \pm \frac{1}{V(y, v, \tau, \varepsilon)}=\left(2\left(h^{ \pm}-\int_{0}^{y} Q_{0}(z, \varepsilon)\right)\right)^{-1 / 2},\left.\quad t^{ \pm}\right|_{y=0}=\tau
\end{array}\right.
$$

para $0 \leq y \leq Z^{ \pm}(v, \tau, \varepsilon)$. As funções $Z^{ \pm}(v, \tau, \varepsilon)$ são contínuas em todas as variáveis.

Dessa forma, $h^{ \pm}$e $t^{ \pm}$são funções contínuas em todas as variáveis, para $\varepsilon=0$ o sistema (130) transforma-se em 


$$
\frac{\partial h^{ \pm}}{\partial y}=0, \quad \frac{\partial t^{ \pm}}{\partial y}= \pm\left[2\left(h-\int_{0}^{y} Q(z) d z\right)\right]^{-1 / 2}
$$

e com isso

$$
h^{ \pm}=\frac{v^{2}}{2}, \quad \tau+\int_{0}^{y} \frac{1}{V(\sigma)} d \sigma=t^{ \pm}(y, \tau)
$$

Para este caso, as funções $Z^{ \pm}(v, \tau, \varepsilon)$ são encontradas de

$$
h=\frac{v^{2}}{2}=\int_{0}^{Z(v)} Q_{0}(z) d z
$$

ou ambas iguais a infinito quando

$$
\frac{v^{2}}{2} \geq \int_{0}^{+\infty} Q_{0}(z) d z
$$

Assim, $Z(v)$ é independente de $\tau$ e dos símobolos \pm . Quando $\varepsilon \rightarrow 0$, temos que $t^{ \pm}(y, v, \tau, \varepsilon) \rightarrow$ $t^{ \pm}(y, v, \tau)$ uniformemente na região $0 \leq y<Z(v)$.

De acordo com

$$
\begin{gathered}
h^{ \pm}(v, \tau, \varepsilon)=\frac{v^{2}}{2}+\int_{0}^{ \pm Z(v, \tau, \varepsilon)}\left[Q_{0}(y, \varepsilon)-Q\left(y, t^{ \pm}(y, v, \tau, \varepsilon)\right)\right] d y= \\
\frac{v^{2}}{2} \varepsilon \int_{0}^{Z^{ \pm}(v, \tau, \varepsilon)}\left[\widetilde{Q}_{0}(y, \varepsilon)-\widetilde{Q}\left(y, t^{ \pm}(y, v, \tau, \varepsilon), \varepsilon\right)\right] d y
\end{gathered}
$$

onde

$$
\widetilde{Q}_{0}(y, \varepsilon)=\frac{1}{2 \pi} \int_{0}^{2 \pi} \widetilde{Q}(y, t, \varepsilon) d t
$$

Nós colocamos

$$
I^{ \pm}=\int_{0}^{Z^{ \pm}(v, \tau, \varepsilon)}\left[\widetilde{Q}_{0}(y, \varepsilon)-\widetilde{Q}\left(y, t^{ \pm}(y, v, \tau, \varepsilon), \varepsilon\right)\right] d y
$$

Então

$$
I^{ \pm}(v, \tau, 0)=\int_{0}^{Z(v)}\left[Q_{10}(y)-Q_{1}\left(y, t^{ \pm}(y, \tau)\right)\right] d y
$$

Da hipótese sobre analiticidade em $\varepsilon$ obtemos (127) por expansão de $I^{ \pm}(v, \tau, \varepsilon)$ em torno de $\varepsilon=0$, e conseqüentemente a continuidade de $O(\varepsilon)$.

Lembremos aqui algumas estimativas importantes:

Se $z^{*} \leq z_{0}, z_{1} \leq z_{2} \leq Z^{ \pm}(v, \tau)$, o número $z^{*}$ é o número definido em $5^{\circ}$ então 


$$
\int_{z_{0}}^{z_{2}} \psi(s) \int_{z_{1}}^{s} \frac{d \sigma d s}{V(\sigma, v, \tau)} \leq\left[\max _{z_{0} \leq s \leq z_{2}} \xi(s)\right]\left[2 \int_{z_{0}}^{Z(v)} q(\sigma) d \sigma\right]
$$

Lembremos também que por (88) temos que

$$
(\delta h)^{\prime}=-Q_{t}^{\prime}(y, t) \delta t, \quad(\delta t)^{\prime}=-\frac{\delta h}{V^{3}}
$$

Observamos que $\frac{\partial h^{+}}{\partial v}$ é solução de (88) com condições iniciais

$$
\delta h(0)=v, \quad \delta t(0)=0
$$

daí por (91) segue que

$$
|\delta t| \leq v \int_{0}^{y} \frac{d s}{V^{3}(s, v, \tau, \varepsilon)} \exp \left\{\varepsilon \int_{0}^{y} \psi(s) d s \int_{0}^{s} \frac{d \sigma}{V^{3}(s, v, \tau, \varepsilon)}\right\} \leq C_{1} \int_{0}^{y} \frac{d s}{V^{3}} .
$$

Aplicando o teorema fundamental do cálculo a (88), juntamente com a desigualdade acima e as condições $4^{\circ}$ e $4^{\text {oo }}$ segue que

$$
\left|\frac{\partial h^{+}}{\partial v}-v\right| \leq \int_{0}^{Z^{+}}\left|Q_{t}^{\prime}\right||\delta(t)| d s \leq \varepsilon C_{1} \int_{0}^{Z^{+}} \psi(s) \int_{0}^{y} \frac{d s d y}{V^{3}(s, v, \tau, \varepsilon)} \leq C_{2} \varepsilon
$$

Uma estimativa análoga se verifica para $\frac{\partial h^{-}}{\partial v}$, o que prova (128).

Para provar (129) observemos que $\frac{\partial h^{+}}{\partial \tau}$ é solução de (88) com condições iniciais

$$
\delta h(0)=0, \quad \delta t(0)=1
$$

Assim por (90), que é relembrada abaixo

$$
\begin{aligned}
& |\delta h(y)| \leq\left[|\delta h(0)|+|\delta t(0)| \int_{0}^{y} \psi(s) d s\right] \exp \int_{0}^{y} \int_{0}^{s} \frac{\psi(s) d s d \sigma}{V^{3}(\sigma)} \Rightarrow \\
& \Rightarrow|\delta h(y)| \leq\left[+\varepsilon \int_{0}^{Z^{+}} \psi(s) d s\right] \exp \varepsilon \int_{0}^{Z^{+}} \psi(s) \int_{0}^{s} \frac{d s d \sigma}{V^{3}(\sigma)} \leq C_{3} \varepsilon
\end{aligned}
$$

Temos ainda que

$$
\frac{\partial Q}{\partial t}=\varepsilon \frac{\partial \widetilde{Q}}{\partial t}=\varepsilon Q_{t}^{\prime}
$$


usando essa informação e (132) segue que

$$
\begin{aligned}
& \frac{1}{\varepsilon} \frac{\partial h^{+}}{\partial \tau}=\int_{0}^{Z^{+}} \frac{Q_{t}^{\prime}}{\varepsilon}(z, t, \varepsilon) \delta t d z=\int_{0}^{Z^{+}} \frac{Q_{t}^{\prime}}{\varepsilon}(z, t, \varepsilon)\left[1-\int_{0}^{Z^{+}} \frac{\delta h^{+}}{V^{3}(s)} d s\right] d z \Rightarrow \\
& \Rightarrow\left|\frac{1}{\varepsilon} \frac{\partial h^{+}}{\partial \tau}-\int_{0}^{Z^{+}} \frac{Q_{t}^{\prime}}{\varepsilon}(z, t, \varepsilon) d z\right| \leq \int_{0}^{Z^{+}} \psi(y) \int_{0}^{y} \frac{C_{3} \varepsilon}{V^{3}(s)} d s d x \leq C_{4} \varepsilon \Rightarrow \\
& \Rightarrow\left|\frac{\partial h^{+}}{\partial \tau}-\int_{0}^{Z^{+}} Q_{t}^{\prime}(z, t, \varepsilon) d z\right| \leq C_{4} \varepsilon^{2} \Rightarrow\left|\frac{\partial h^{+}}{\partial \tau}-\varepsilon \int_{0}^{Z^{+}} \widetilde{Q}_{t}^{\prime}(z, t, \varepsilon)\right| \leq C_{4} \varepsilon^{2}
\end{aligned}
$$

Conseqüentemente (129) decorre do desenvolvimente em torno de $\varepsilon=0$ da exrpessão

$$
\int_{0}^{Z^{+}(v, \tau, \varepsilon)} \widetilde{Q}(z, t, \varepsilon) d z
$$

A demonstração de (129) para $\frac{\partial h^{-}}{\partial \tau}$ é análoga à que acabamos de mostrar.

Corolário 4.3. Se as condições sobre $Q(z, t)$ da proposição anterior são verificadas, então para $\varepsilon$ suficientemente pequeno cada ponto regular de $\left(v_{0}, \tau_{0}\right) \in \Pi_{0}^{+} \cap \Pi_{0}^{-}$, está contido numa vizinhança pequena de $\left(\left[\int_{0}^{+\infty} Q(z) d z\right]^{1 / 2}, \tau_{0}\right)$, e corresponde a um zero simples $\tau_{0}$ da função

$$
\left.A(\tau)=\int_{-\infty}^{+\infty} Q_{1}\left(y, \tau+\left[\int_{0}^{y} v^{2}-2 \int_{0}^{+\infty} Q(z) d z\right)\right]^{-1 / 2}\right) d y
$$

(a integral imprópria aqui é tomada como sendo o valor principal de Cauchy)

Demonstração. Os pontos de $\Pi_{0}^{+} \cap \Pi_{0}^{-}$são encontrados a partir do sistema

$$
h^{+}(v, \tau, \varepsilon)=h^{-}(v, \tau, \varepsilon)=\int_{0}^{+\infty} Q_{0}(z, \varepsilon) d z
$$

que é equivalente a

$$
\frac{h^{+}(v, \tau, \varepsilon)-h^{-}(v, \tau, \varepsilon)}{\varepsilon}=0, h^{+}(v, \tau, \varepsilon)=\int_{0}^{+\infty} Q_{0}(z, \varepsilon) d z
$$

Se usarmos (127) combinado a (134), quando fazemos $\varepsilon \rightarrow 0$ o sistema (135) temos

$$
\begin{gathered}
\int_{0}^{+\infty} Q_{0}(z) d z=h^{+}(v, \tau)=h^{+}(v, \tau, 0)= \\
\lim _{\varepsilon \rightarrow 0} h^{+}(v, \tau, \varepsilon)=\lim _{\varepsilon \rightarrow 0} \frac{v^{2}}{2}+\varepsilon \int_{0}^{X(v)}\left[Q_{1,0}(y)-Q_{1}\left(y, t^{ \pm}(y, \tau)\right)\right] d y+O(\varepsilon)=\frac{v^{2}}{2}
\end{gathered}
$$


e também temos

$$
\begin{aligned}
\frac{h^{+}(v, \tau, \varepsilon)-h^{-}(v, \tau, \varepsilon)}{\varepsilon}=0 & \Rightarrow \int_{0}^{+\infty}\left[Q_{1,0}(y)-Q_{1}\left(y, t^{+}(y, \tau)\right)\right] d y= \\
& =\int_{0}^{+\infty}\left[Q_{1,0}(y)-Q_{1}\left(y, t^{-}(y, \tau)\right)\right] d y
\end{aligned}
$$

quando $\varepsilon \rightarrow 0$

Assim, o sistema (135) ganha a forma

$$
\begin{gathered}
\frac{v^{2}}{2}=\int_{0}^{+\infty} Q_{0}(z) d z \\
\int_{0}^{+\infty}\left[Q_{1,0}(y)-Q_{1}\left(y, t^{+}(y, \tau)\right)\right] d y=\int_{0}^{+\infty}\left[Q_{1,0}(y)-Q_{1}\left(y, t^{-}(y, \tau)\right)\right] d y
\end{gathered}
$$

Das simetrias do problema temos que $t^{+}(y, v, \tau)=t^{-}(-y, v, \tau)=t^{+}(-y,-v, \tau)$ o qual se obtemos resolvendo a equação abaixo na variável $t$

$$
z\left(t, v^{\prime}, \tau^{\prime}\right)=-y
$$

que é equivalente a

$$
z\left(t^{\prime}, v^{\prime}, \tau^{\prime}\right)=z(t,-v, \tau)-z(t, v, \tau)=-y \Rightarrow z(t, v, \tau)=y
$$

Como $Q_{1}$ é ímpar em z, podemos trocar (137) por

$$
\int_{-\infty}^{+\infty}\left[Q_{1}\left(y+t^{+}(y, v, \tau)\right)\right] d y
$$

Lembrando que

$$
t^{+}(y, v, \tau)=\tau+\int_{0}^{t} \frac{1}{V(\sigma, v, \tau)} d \sigma
$$

e

$$
V^{2}(\sigma)=v^{2}-2 \int_{0}^{\sigma} Q(z) d z
$$

Substituindo convenientemente essas duas expressões em (138) obtemos o desejado. Agora, usando as expressões para as derivadas parciais $\frac{\partial h^{ \pm}}{\partial v}$ e $\frac{\partial h^{ \pm}}{\partial \tau}$ dadas em (128) e (129) e supondo $\tau_{0}$ um zero simples de $A(\tau)$ temos 


$$
\frac{\partial\left(h^{-}, h^{+}\right)}{\partial(v, \tau)}=\left|\begin{array}{cc}
v+O(\varepsilon) & \varepsilon \int_{0}^{+\infty} Q_{1, t}^{\prime}\left(y, t^{-}(y, v, \tau)\right) d y+o(\varepsilon) \\
v+O(\varepsilon) & \varepsilon \int_{0}^{+\infty} Q_{1, t}^{\prime}\left(y, t^{+}(y, v, \tau)\right) d y+o(\varepsilon)
\end{array}\right|=v \varepsilon A^{\prime}\left(\tau_{0}\right)+O(\varepsilon) \neq 0
$$

Se $\varepsilon$ é suficientemente pequeno. Daí o ponto encontrado em $\Pi_{0}^{+} \cap \Pi_{0}^{-}$é regular. 


\section{Referências}

[1] V.M. Alekseev, Quasirandom Dynamical Systems. I,II,III, English trasl. in Math USSR Sb. 5(1968),6(1968), 7(1969).

[2] A.G Kušnirenko, A.B. Katok, V.M. Alekseev, Three Papers on Dynamical Systems vol. 116, American Mathematical Society, 1981.

[3] Jürgen Moser, Stable and random motions in dynamical systems, Princeton Univ. Press, Princeton, 1973.

[4] V.I. Arnold, Mathematical Methods of Classical Mechanics. SpringerVerlag, New York, second edition, 1989.

[5] V.I. Arnold, Métodos Matemáticos da Mecânica Clássica. Mir Moscovo, Rio de Janeiro, 1987.

[6] Ricardo Mañé, Teoria Ergódica, Publicação IMPA, Rio de Janeiro, 1983.

[7] R. McGehee, A stable manifold theorem for degenerate fixed points with Aplications to Celestial Mechanics, Journal of Differential Equations 14 (1973), 70-88.

[8] J. Guckenheimer e Paul Holmes, Nonlinear Oscilations, Dynamical Systems, and Bifurcations of Vector Fields, Springer-Verlag, New Yorke, 1983.

[9] C. Robinson, Introduction to the Theory of Dynamical Systems, CRC Press, Boca Raton, 1995.

[10] Harry Dankowicz e Philip Holmes, The Existence of Transverse Homoclinic Points in the Sitnikov Problem, Journal of Differential Equations 116 (1993),468-483. 